\title{
Photoelectrocyclization Reactions of Amidonaphthoquinones
}

Jinya Yin, Michael B. Landward, and Jon D. Rainier* 315 South, 1400 East

Department of Chemistry

$$
\text { University of Utah }
$$

Salt Lake City, UT 84112

email: rainier@chem.utah.edu

Contents

1. 5 ( ${ }^{1} \mathrm{H}$ NMR)

2. $5\left({ }^{13} \mathrm{C}\right.$ NMR $)$

3. S1 ( ${ }^{1} \mathrm{H}$ NMR)

4. $\mathrm{S} 1$ ( ${ }^{13} \mathrm{C}$ NMR)

5. 6 ( ${ }^{1} \mathrm{H}$ NMR)

6. $6\left({ }^{13} \mathrm{C} \mathrm{NMR}\right)$

7. S2 ( ${ }^{1} \mathrm{H}$ NMR)

8. S2 ( ${ }^{13} \mathrm{C}$ NMR)

9. 7 ( ${ }^{1} \mathrm{H}$ NMR)

10. 7 ( ${ }^{13} \mathrm{C}$ NMR $)$

11.8 ( ${ }^{1} \mathrm{H}$ NMR)

$12.8\left({ }^{13} \mathrm{C}\right.$ NMR $)$

13.9 ( ${ }^{1} \mathrm{H}$ NMR)

14. $9\left({ }^{13} \mathrm{C}\right.$ NMR $)$

15. S3 ( ${ }^{1} \mathrm{H}$ NMR)

16. S3 ( ${ }^{13} \mathrm{C}$ NMR $)$

17. 10 ( ${ }^{1} \mathrm{H}$ NMR)

18. 10 ( ${ }^{13} \mathrm{C}$ NMR $)$

19. 11 ( $\left.{ }^{1} \mathrm{H} \mathrm{NMR}\right)$

20. $11\left({ }^{13} \mathrm{C}\right.$ NMR $)$

21. $12\left({ }^{1} \mathrm{H}\right.$ NMR)

22. $12\left({ }^{13} \mathrm{C}\right.$ NMR $)$

23. S4 ( ${ }^{1} \mathrm{H}$ NMR)

24. S4 ( ${ }^{13} \mathrm{C}$ NMR $)$

25. 13 ( ${ }^{1} \mathrm{H}$ NMR)

26. $13\left({ }^{13} \mathrm{C}\right.$ NMR $)$

27. 14 ( ${ }^{1} \mathrm{H}$ NMR)

28. $14\left({ }^{13} \mathrm{C}\right.$ NMR $)$

29. S5 ( ${ }^{1} \mathrm{H}$ NMR)

30. S5 ( ${ }^{13} \mathrm{C}$ NMR)

31. 16 ( ${ }^{1} \mathrm{H}$ NMR)

$32.16\left({ }^{13} \mathrm{C}\right.$ NMR $)$

33. 17 ( ${ }^{1} \mathrm{H}$ NMR)

34. $17\left({ }^{13} \mathrm{C}\right.$ NMR $)$

35. 19 ( ${ }^{1} \mathrm{H}$ NMR)

36. $19\left({ }^{13} \mathrm{C}\right.$ NMR $)$

37. 20 ( ${ }^{1} \mathrm{H}$ NMR)

38. 20 ( ${ }^{13} \mathrm{C}$ NMR)

39. 22 ( ${ }^{1} \mathrm{H}$ NMR)

40. $22\left({ }^{13} \mathrm{C}\right.$ NMR $)$

41. 23 ( ${ }^{1} \mathrm{H}$ NMR)

42. 23 ( ${ }^{13} \mathrm{C}$ NMR $)$
43. S6 $\left({ }^{1} \mathrm{H}\right.$ NMR $) \quad \mathrm{S} 43$

44. S6 $\left({ }^{13} \mathrm{C}\right.$ NMR $) \quad \mathrm{S} 44$

45. 29 ( ${ }^{1} \mathrm{H}$ NMR) $\quad \mathrm{S} 45$

46. $29\left({ }^{13} \mathrm{C}\right.$ NMR $) \quad \mathrm{S} 46$

47. 30 ( ${ }^{1} \mathrm{H}$ NMR) $\quad \mathrm{S} 47$

48. 30 ( ${ }^{13} \mathrm{C}$ NMR $) \quad \mathrm{S} 48$

49. S7 ( ${ }^{1} \mathrm{H}$ NMR) $\quad \mathrm{S} 49$

50. S7 $\left({ }^{13} \mathrm{C}\right.$ NMR $) \quad \mathrm{S} 50$

51.31 ( ${ }^{1} \mathrm{H}$ NMR) $\quad \mathrm{S} 51$

52. 31 ( ${ }^{13} \mathrm{C}$ NMR) $\quad \mathrm{S} 52$

53. 32 ( ${ }^{1} \mathrm{H}$ NMR) $\quad \mathrm{S} 53$

54. 32 ( ${ }^{13} \mathrm{C}$ NMR) $\quad \mathrm{S} 54$

55. S8 $\left({ }^{1} \mathrm{H}\right.$ NMR) $\quad \mathrm{S} 55$

56. S8 $\left({ }^{13} \mathrm{C}\right.$ NMR $) \quad \mathrm{S} 56$

$57.33\left({ }^{1} \mathrm{H}\right.$ NMR) $\quad \mathrm{S} 57$

$58.33\left({ }^{13} \mathrm{C}\right.$ NMR $) \quad \mathrm{S} 58$

59. $34\left({ }^{1} \mathrm{H}\right.$ NMR $) \quad$ S59

$60.34\left({ }^{13} \mathrm{C}\right.$ NMR $) \quad \mathrm{S} 60$

61. S9 ( ${ }^{1} \mathrm{H}$ NMR) $\quad$ S61

62. S9 $\left({ }^{13} \mathrm{C}\right.$ NMR) $\quad \mathrm{S} 62$

63. $35\left({ }^{1} \mathrm{H}\right.$ NMR $) \quad \mathrm{S} 63$

64. $35\left({ }^{13} \mathrm{C}\right.$ NMR $) \quad \mathrm{S} 64$

$65.36\left({ }^{1} \mathrm{H}\right.$ NMR $) \quad \mathrm{S} 65$

66. $36\left({ }^{13} \mathrm{C}\right.$ NMR $) \quad \mathrm{S} 66$

67. S10 ( ${ }^{1} \mathrm{H}$ NMR) $\quad \mathrm{S} 67$

68. S10 ( ${ }^{13} \mathrm{C}$ NMR) $\quad \mathrm{S} 68$

69. $37\left({ }^{1} \mathrm{H}\right.$ NMR) $\quad$ S69

$70.37\left({ }^{13} \mathrm{C} \mathrm{NMR}\right) \quad \mathrm{S} 70$

71.38 ( ${ }^{1} \mathrm{H}$ NMR $) \quad \mathrm{S} 71$

72. $38\left({ }^{13} \mathrm{C}\right.$ NMR $) \quad \mathrm{S} 72$

73. S11 ( ${ }^{1} \mathrm{H}$ NMR) $\quad \mathrm{S} 73$

74. S11 ( ${ }^{13} \mathrm{C}$ NMR $) \quad \mathrm{S} 74$

75. 39 ( ${ }^{1} \mathrm{H}$ NMR) $\quad \mathrm{S} 75$

76. $39\left({ }^{13} \mathrm{C} \mathrm{NMR}\right) \quad \mathrm{S} 76$

$77.40\left({ }^{1} \mathrm{H}\right.$ NMR $) \quad \mathrm{S} 77$

78. 40 ( ${ }^{13} \mathrm{C}$ NMR $) \quad \mathrm{S} 78$

79. S12 ( ${ }^{1} \mathrm{H}$ NMR) $\quad \mathrm{S} 79$

80. S12 ( ${ }^{13} \mathrm{C}$ NMR $) \quad \mathrm{S} 80$

$81.41\left({ }^{1} \mathrm{H}\right.$ NMR $) \quad \mathrm{S} 81$

82. $41\left({ }^{13} \mathrm{C} \mathrm{NMR}\right) \quad \mathrm{S} 82$

$83.42\left({ }^{1} \mathrm{H}\right.$ NMR) $\quad \mathrm{S} 83$

84. $42\left({ }^{13} \mathrm{C}\right.$ NMR $) \quad \mathrm{S} 84$

$85.43\left({ }^{1} \mathrm{H}\right.$ NMR) $\quad \mathrm{S} 85$ 
86. $43\left({ }^{13} \mathrm{C} \mathrm{NMR}\right) \quad \mathrm{S} 86$

87. S13 $\left({ }^{1} \mathrm{H}\right.$ NMR $) \quad \mathrm{S} 87$

88. S13 $\left({ }^{13} \mathrm{C}\right.$ NMR $) \quad \mathrm{S} 88$

89. $45\left({ }^{1} \mathrm{H}\right.$ NMR $) \quad \mathrm{S} 89$

90. $45\left({ }^{13}\right.$ C NMR $) \quad \mathrm{S} 90$

91. $47\left({ }^{1} \mathrm{H}\right.$ NMR $) \quad \mathrm{S} 91$

92. 47 ( ${ }^{13} \mathrm{C}$ NMR) $\quad \mathrm{S} 92$

93. ( \pm ) Griffithazanone A $\left({ }^{1} \mathrm{H}\right.$ NMR) $\mathrm{S} 93$

94. ( \pm Griffithazanone A $\left({ }^{13} \mathrm{C}\right.$ NMR $)$ S94

95. Fig S1. Spectral distribution and intensity of RPR-4190A S95

96. Fig S2. Crystal structure of $\mathbf{1 4}$ S96

97. Fig S3. Crystal structure of $40 \quad$ S97

98. Methods of XRD sample preparations and XRD determination $\quad$ S98

99. Table S1. Summary of XRD parameters $\begin{array}{ll}\text { of } \mathbf{1 4} \text { and } 40 & \text { S98 }\end{array}$ 


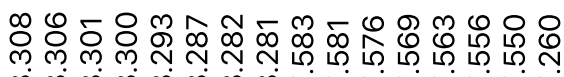

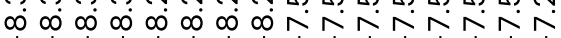

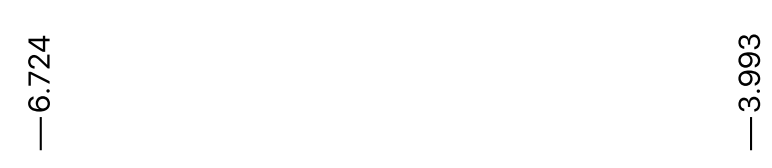

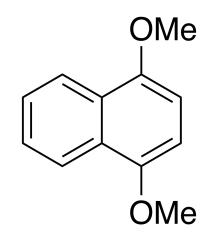

5, ${ }^{1} \mathrm{H}$ NMR $\left(500 \mathrm{MHz}, \mathrm{CDCl}_{3}\right)$

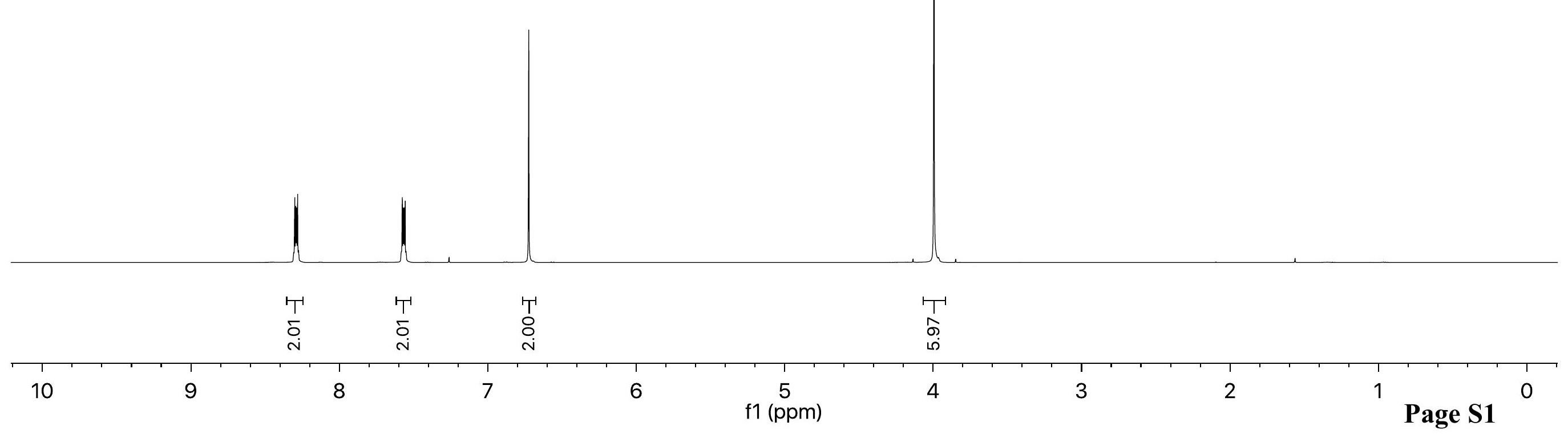




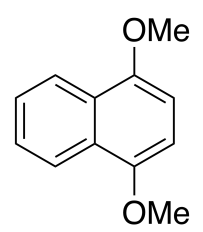

5, ${ }^{13} \mathrm{C}$ NMR $\left(126 \mathrm{MHz}, \mathrm{CDCl}_{3}\right)$

乐

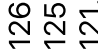

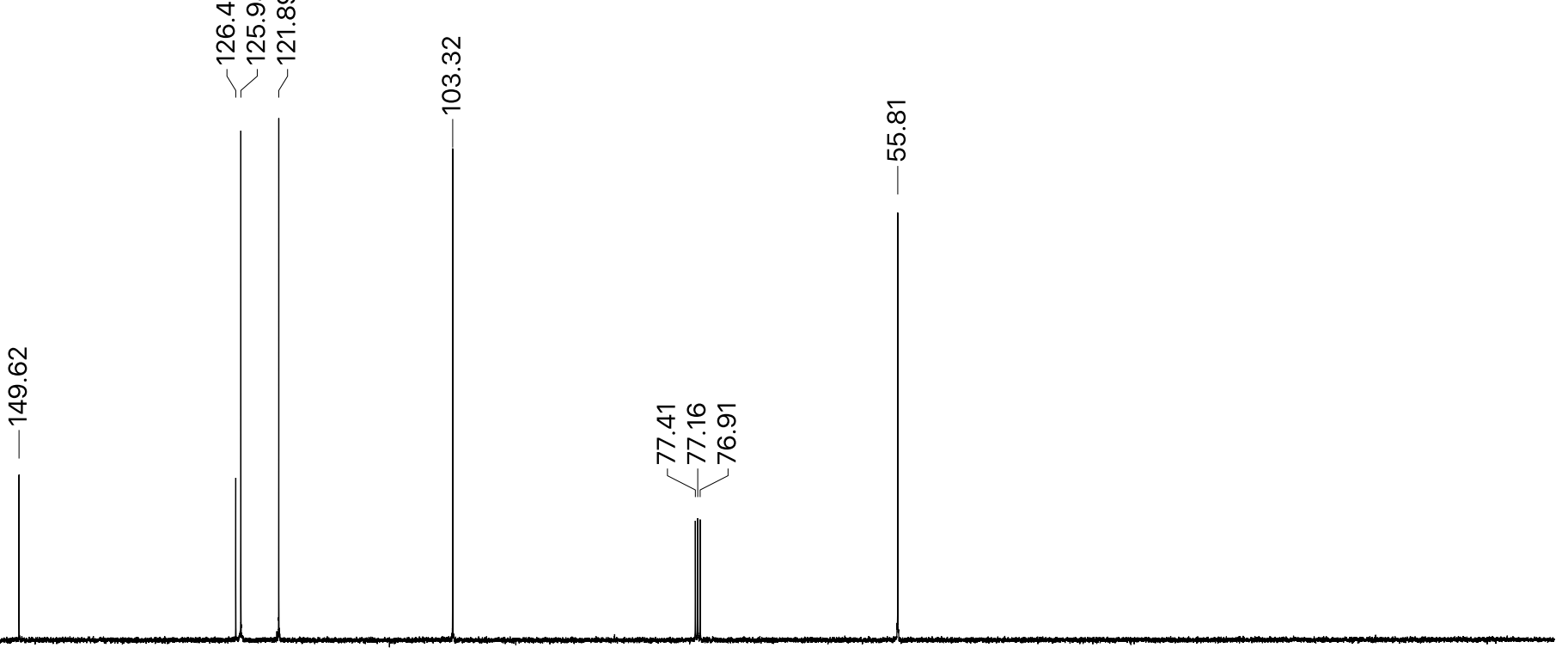


م⿻

$\infty \underbrace{\infty}$

$\mathrm{OMe}$

$\mathrm{OMe}^{\mathrm{NO}_{2}}$

S1, ${ }^{1} \mathrm{H}$ NMR $\left(500 \mathrm{MHz}, \mathrm{CDCl}_{3}\right)$

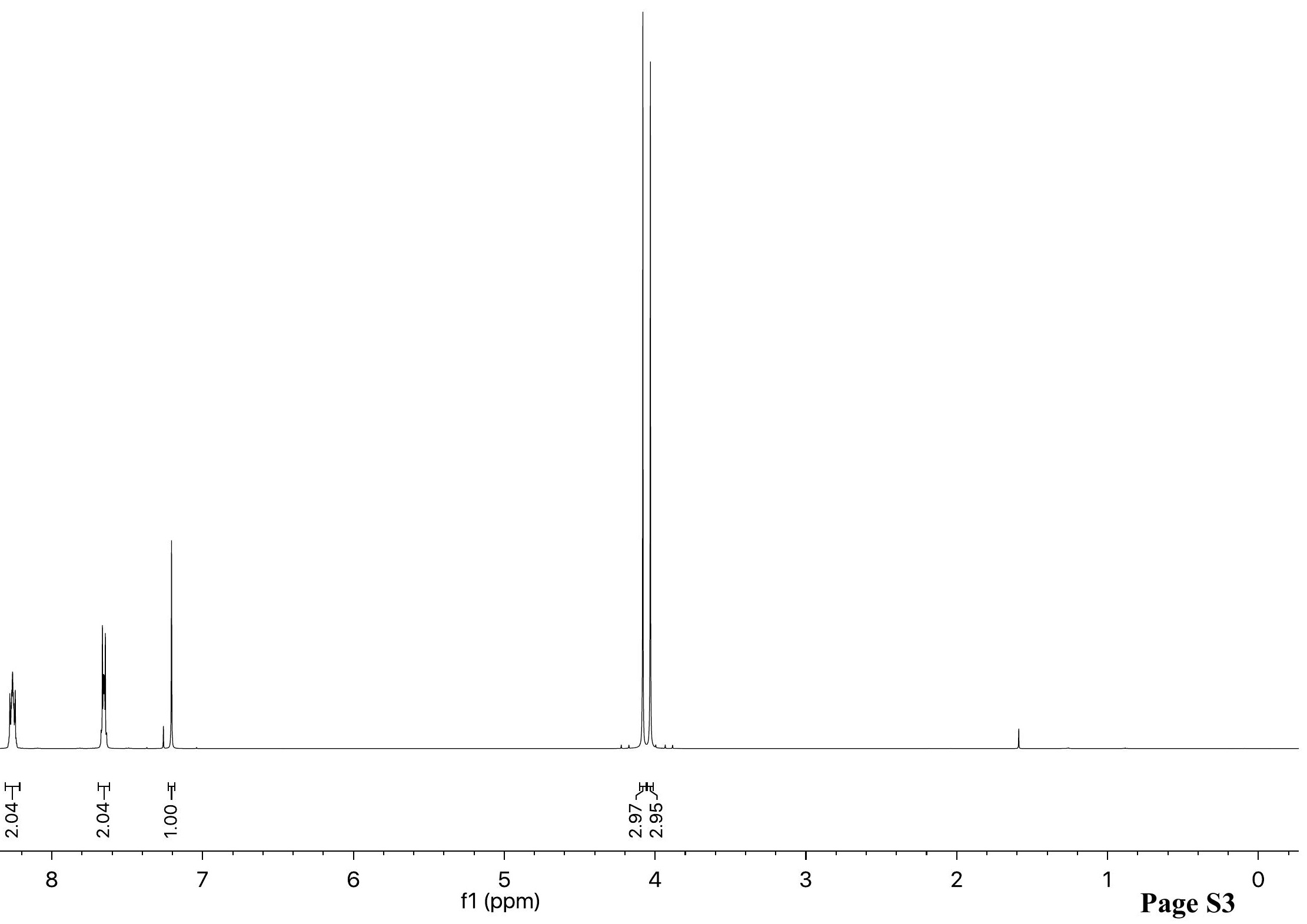




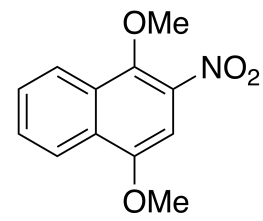

S1, ${ }^{13} \mathrm{C}$ NMR $\left(126 \mathrm{MHz}, \mathrm{CDCl}_{3}\right)$

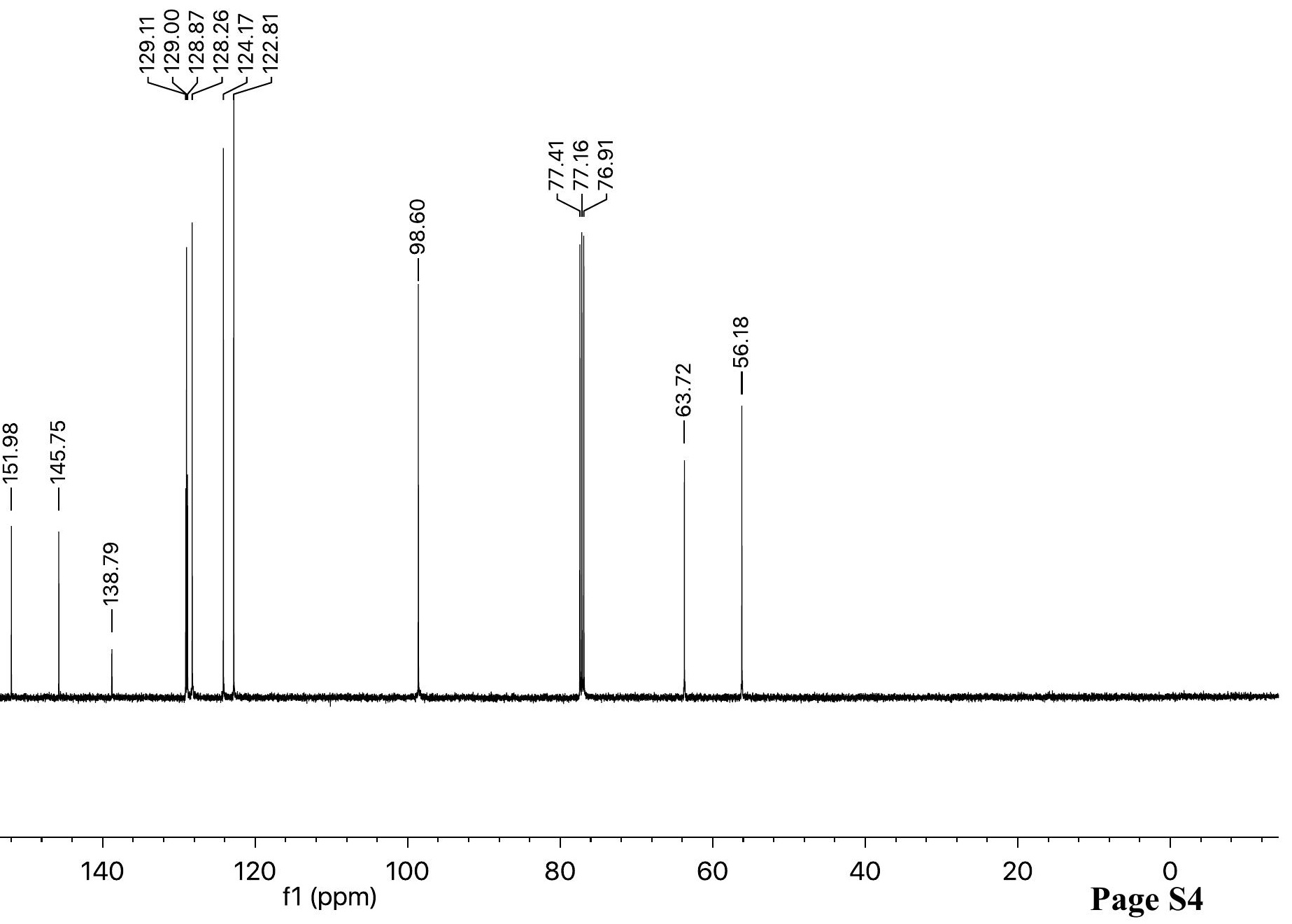




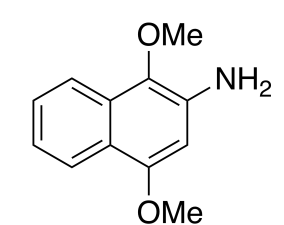

6, ${ }^{1} \mathrm{H}$ NMR $\left(500 \mathrm{MHz}, \mathrm{CDCl}_{3}\right)$

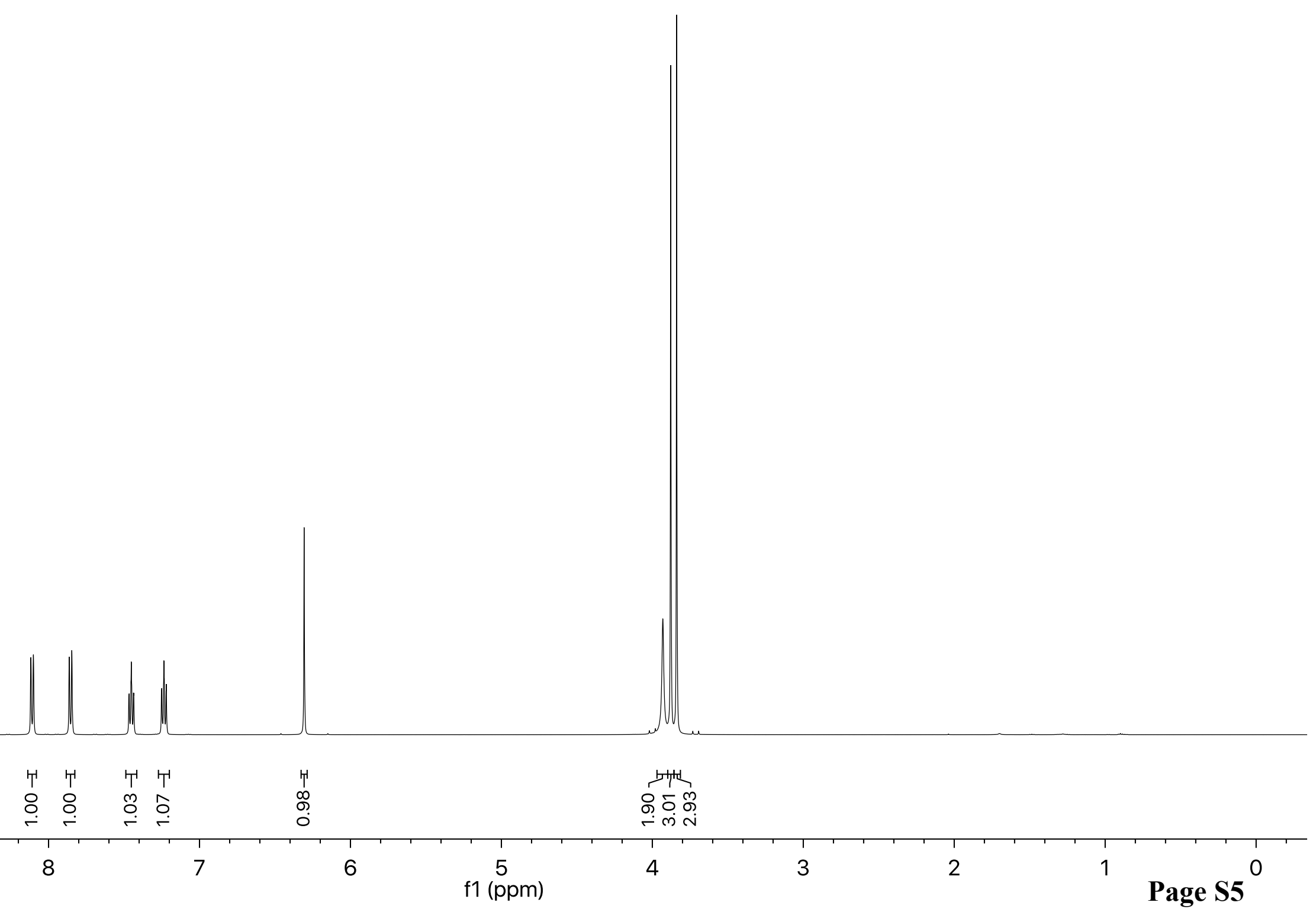




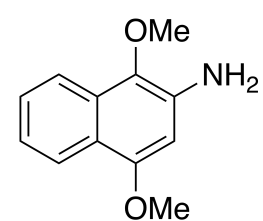

6, ${ }^{13} \mathrm{C}$ NMR $\left(126 \mathrm{MHz}, \mathrm{CDCl}_{3}\right)$

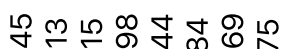

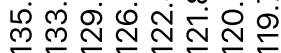

रंर्याi

ó

8 ํํำ

|

긍ำ

소용

กั่

i

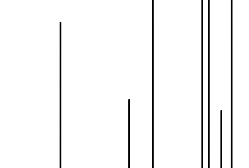

|| $\mid$ 


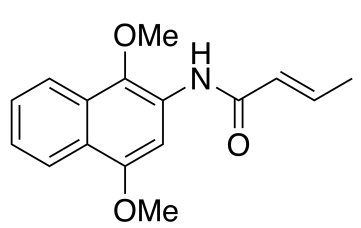

S2, ${ }^{1} \mathrm{H}$ NMR $\left(500 \mathrm{MHz}, \mathrm{CDCl}_{3}\right)$

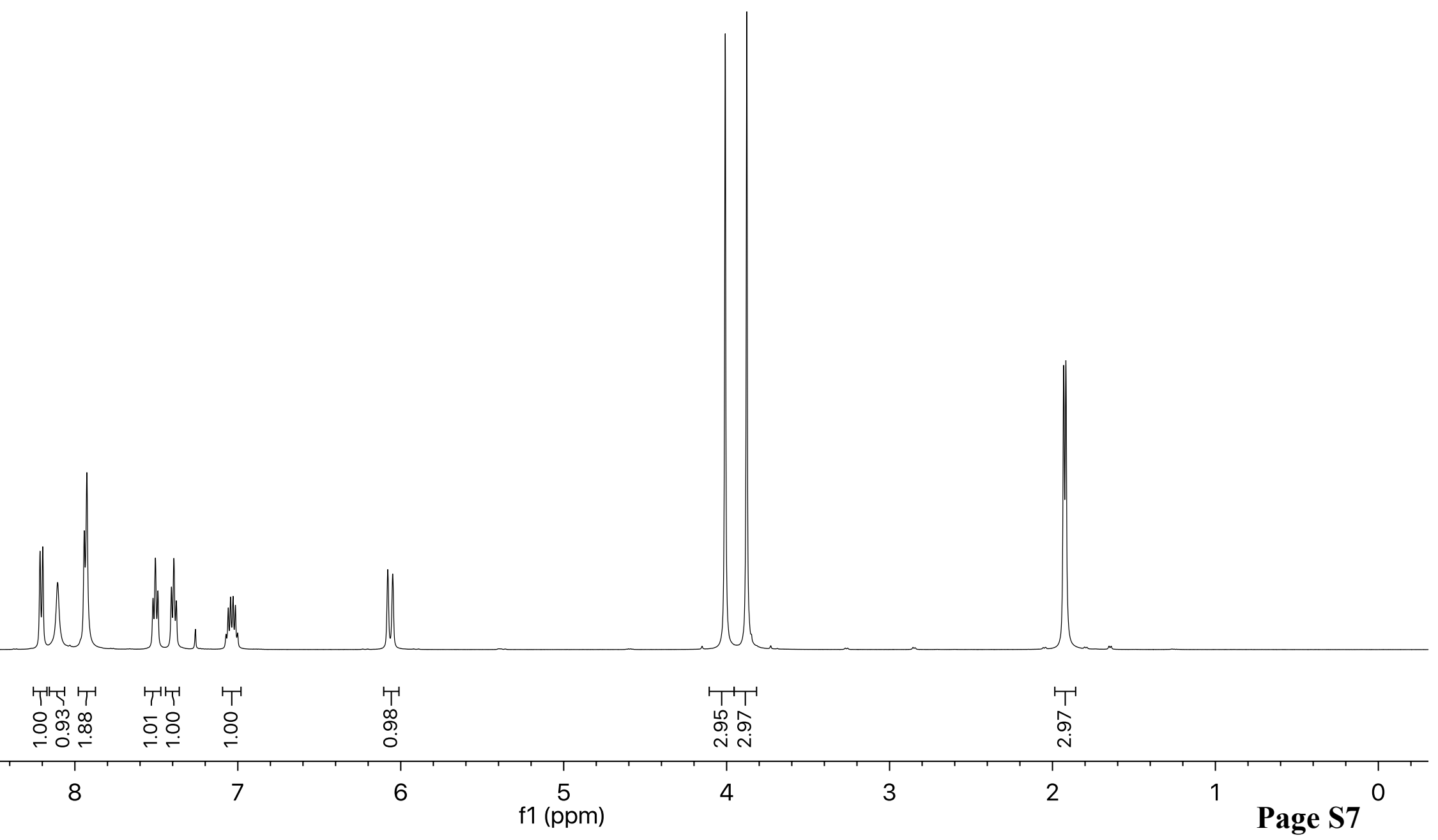




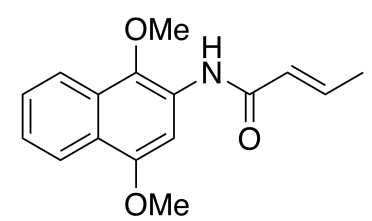

S2, $\left.{ }^{13} \mathrm{C} \mathrm{NMR} \mathrm{(126} \mathrm{MHz,} \mathrm{CDCl}_{3}\right)$

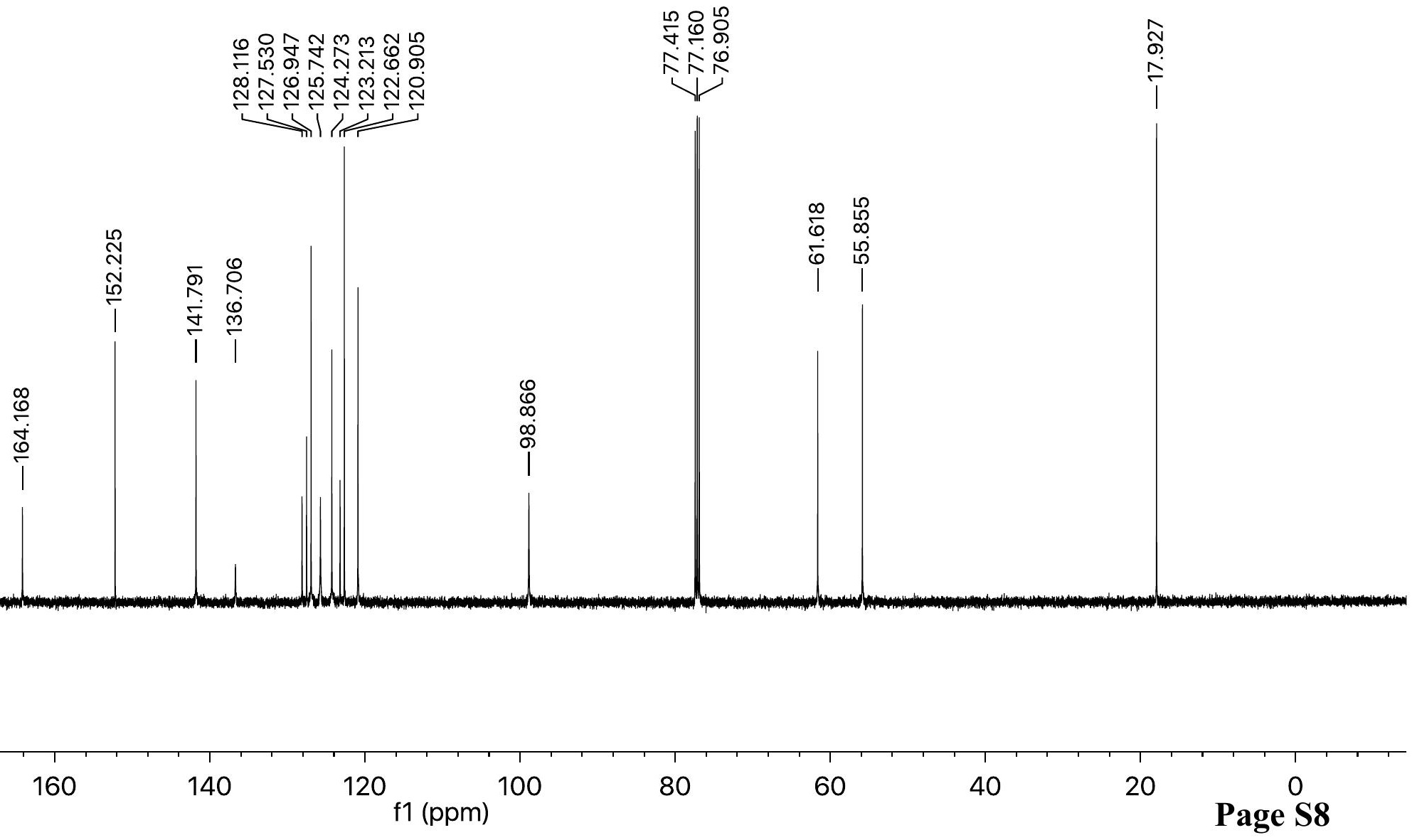




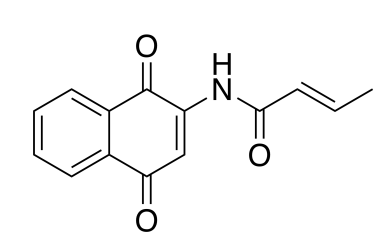

$\begin{array}{llll}\infty & 0 & 0 \\ & 0 & 0 \\ 0 & 0 & 0 \\ 0 & 0 & 0 & 0 \\ 0\end{array}$

7, ${ }^{1} \mathrm{H}$ NMR $\left(500 \mathrm{MHz}, \mathrm{CDCl}_{3}\right)$

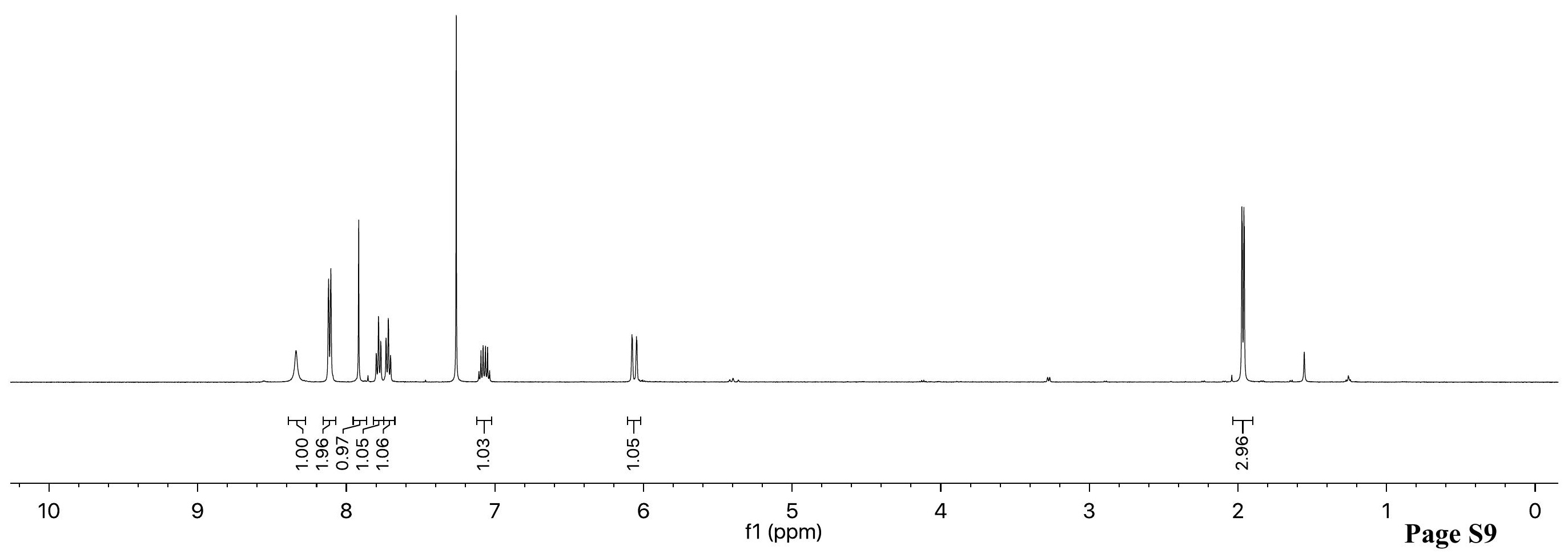




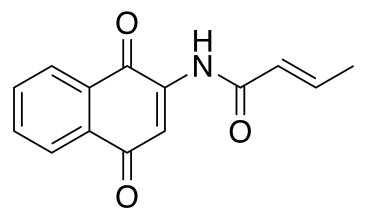

7, ${ }^{13} \mathrm{C} \mathrm{NMR} \mathrm{(126} \mathrm{MHz,} \mathrm{CDCl}_{3}$ )

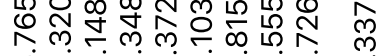

寸乎兽孞

సָำ ำ

$\stackrel{1}{\infty} \stackrel{\infty}{\infty}$

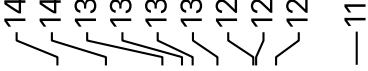

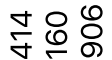

ㅅํㅇ

广

గִ
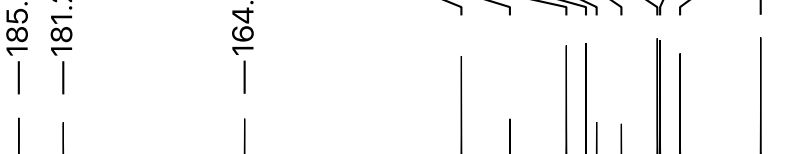


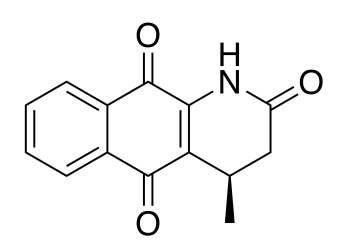

8, ${ }^{1} \mathrm{H}$ NMR $\left(500 \mathrm{MHz}, \mathrm{CDCl}_{3}\right)$

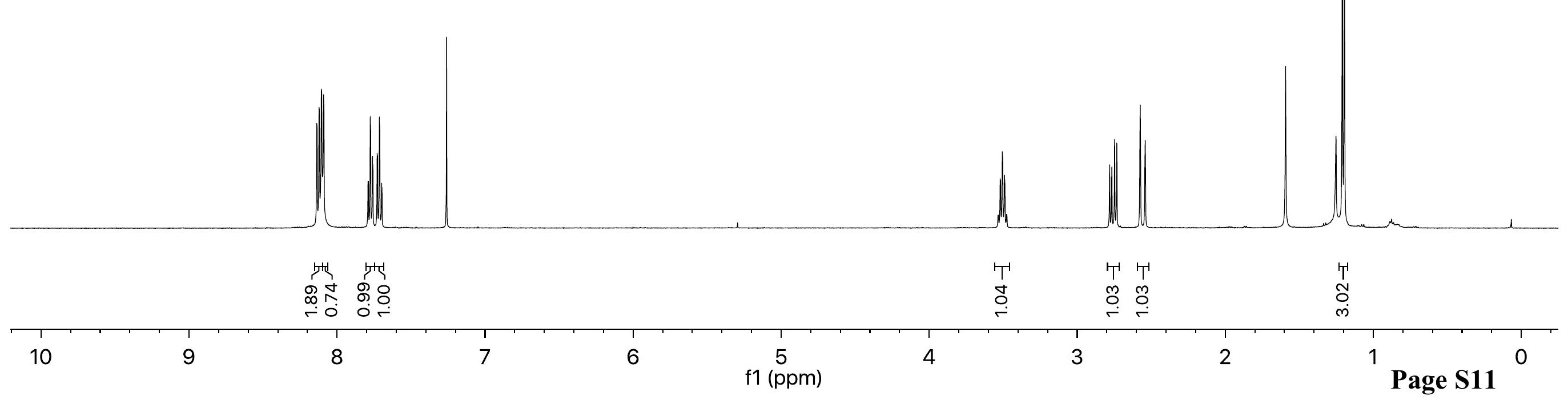




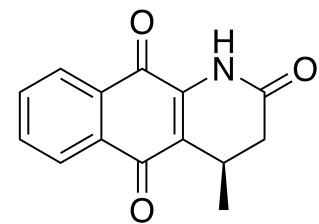

8, ${ }^{13} \mathrm{C}$ NMR $\left(126 \mathrm{MHz}, \mathrm{CDCl}_{3}\right)$

$\dot{7} \div$

र쇼

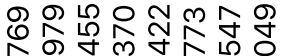

ले

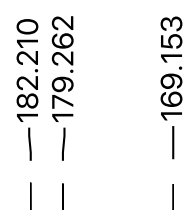

它证

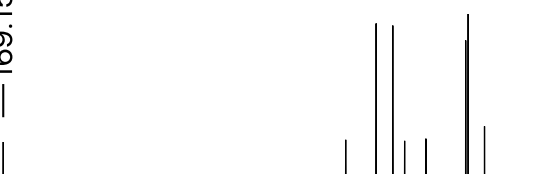


$\underbrace{\circ}$

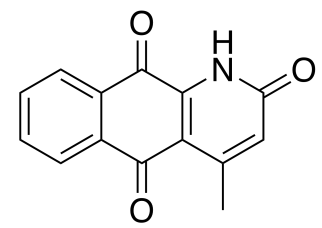

9, ${ }^{1} \mathrm{H}$ NMR $\left(500 \mathrm{MHz}, \mathrm{CDCl}_{3}\right)$

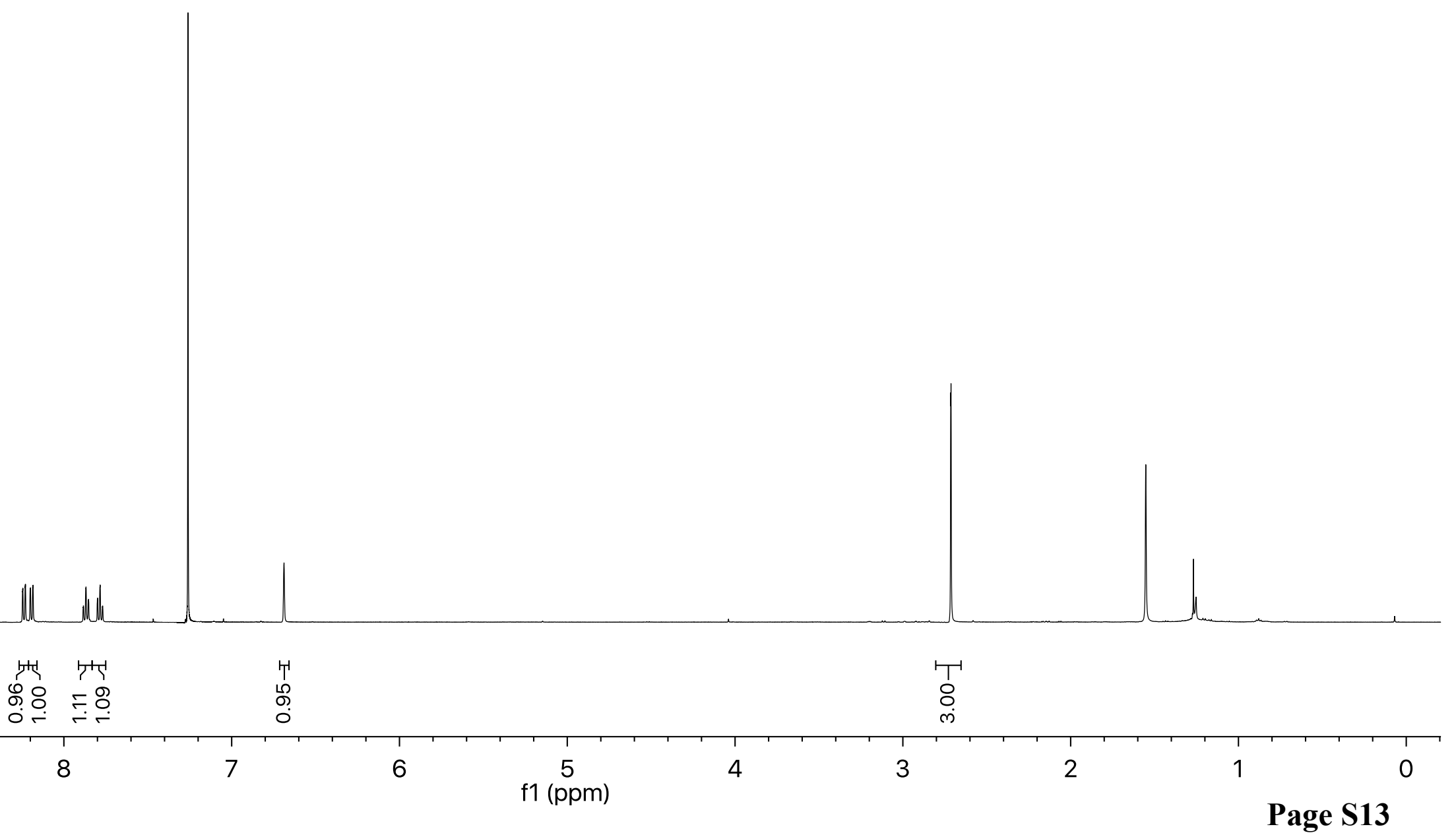




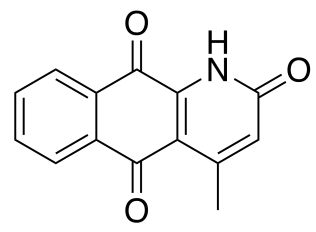

9, ${ }^{13} \mathrm{C}$ NMR $\left(126 \mathrm{MHz}, \mathrm{CDCl}_{3}\right)$

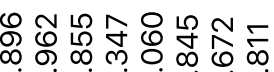

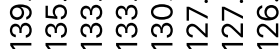

运拧

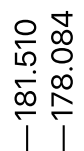

ஸे

듬

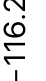




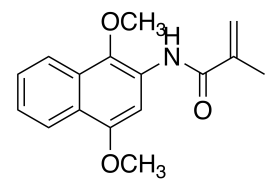

S3, ${ }^{1} \mathrm{H}$ NMR $\left(500 \mathrm{MHz}, \mathrm{CDCl}_{3}\right)$

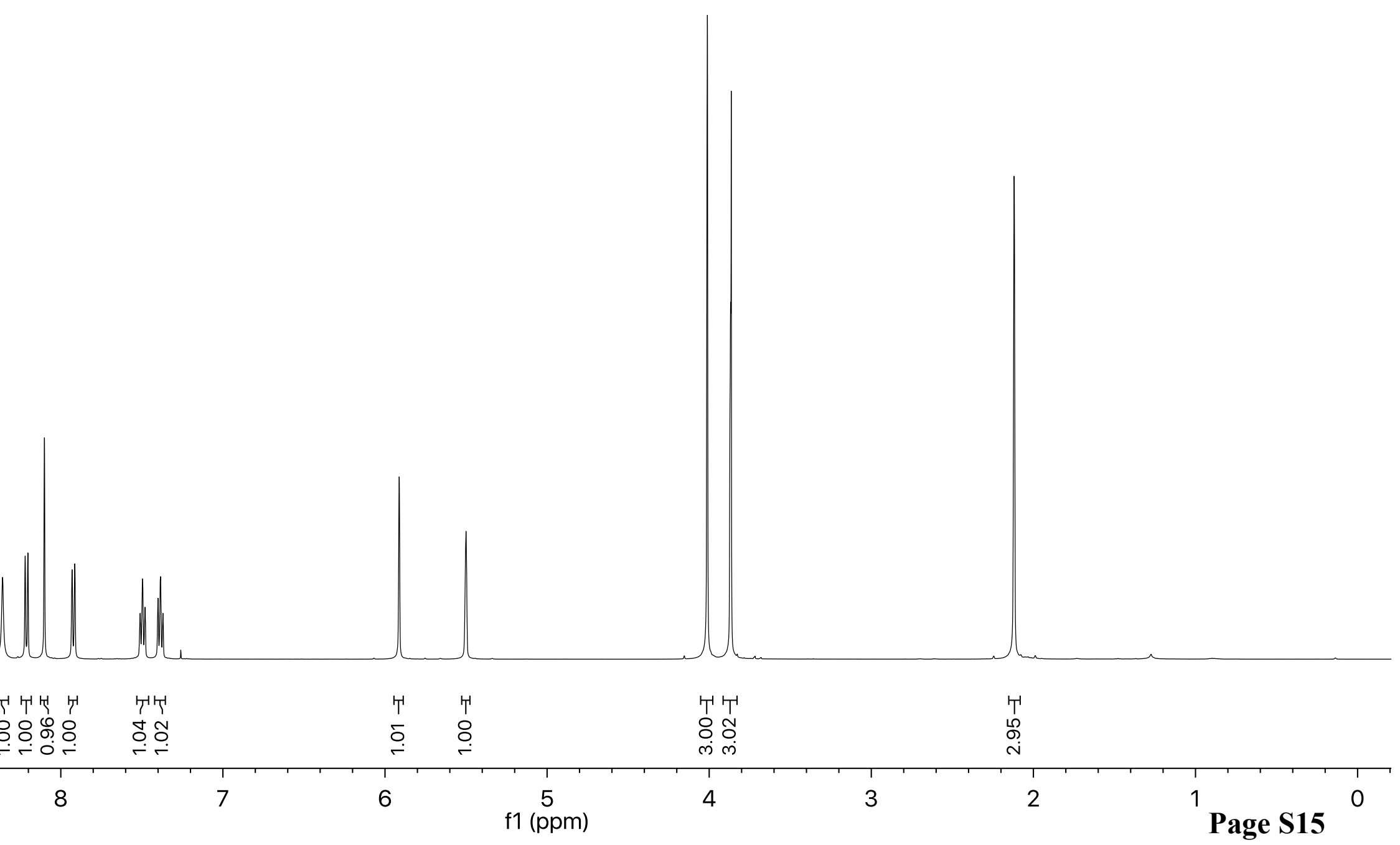




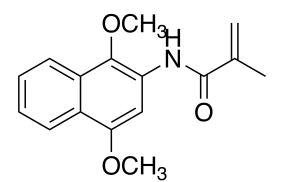

S3, ${ }^{13} \mathrm{C}$ NMR $\left(126 \mathrm{MHz}, \mathrm{CDCl}_{3}\right)$

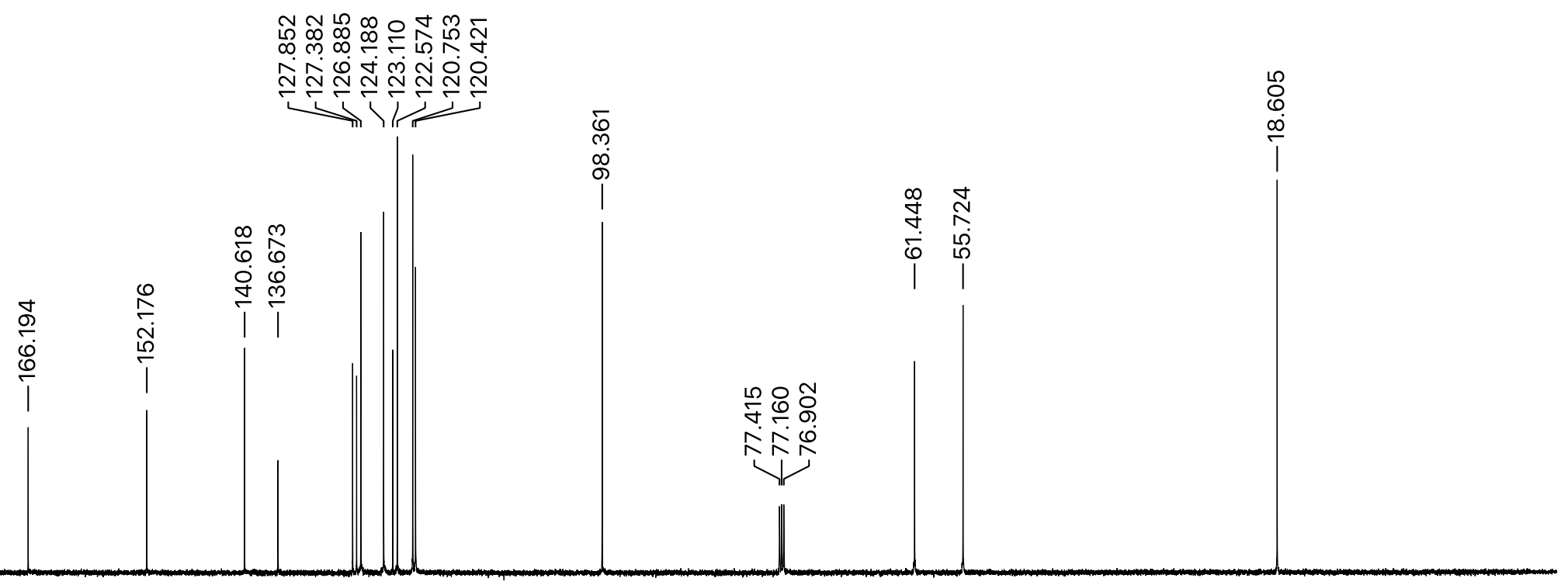

0




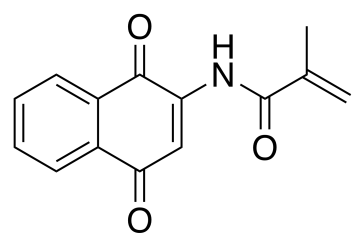

10, ${ }^{1} \mathrm{H}$ NMR $\left(500 \mathrm{MHz}, \mathrm{CDCl}_{3}\right)$

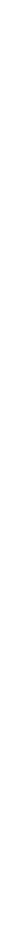




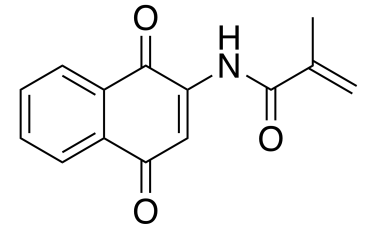

10, $\left.{ }^{13} \mathrm{C} \mathrm{NMR} \mathrm{(126} \mathrm{MHz,} \mathrm{CDCl}_{3}\right)$

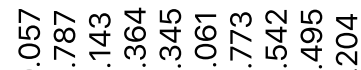

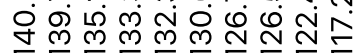

үर

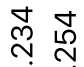

ஸे

$\stackrel{\infty}{\infty}$

\%

$\stackrel{\infty}{0}$

|

$\begin{array}{rll}18 & 1 & 1 \\ 220 & 200\end{array}$




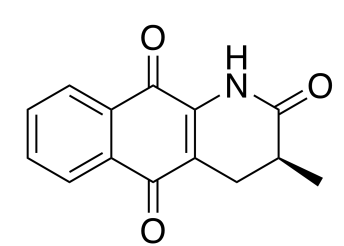

11, ${ }^{1} \mathrm{H}$ NMR $\left(500 \mathrm{MHz}, \mathrm{CDCl}_{3}\right)$

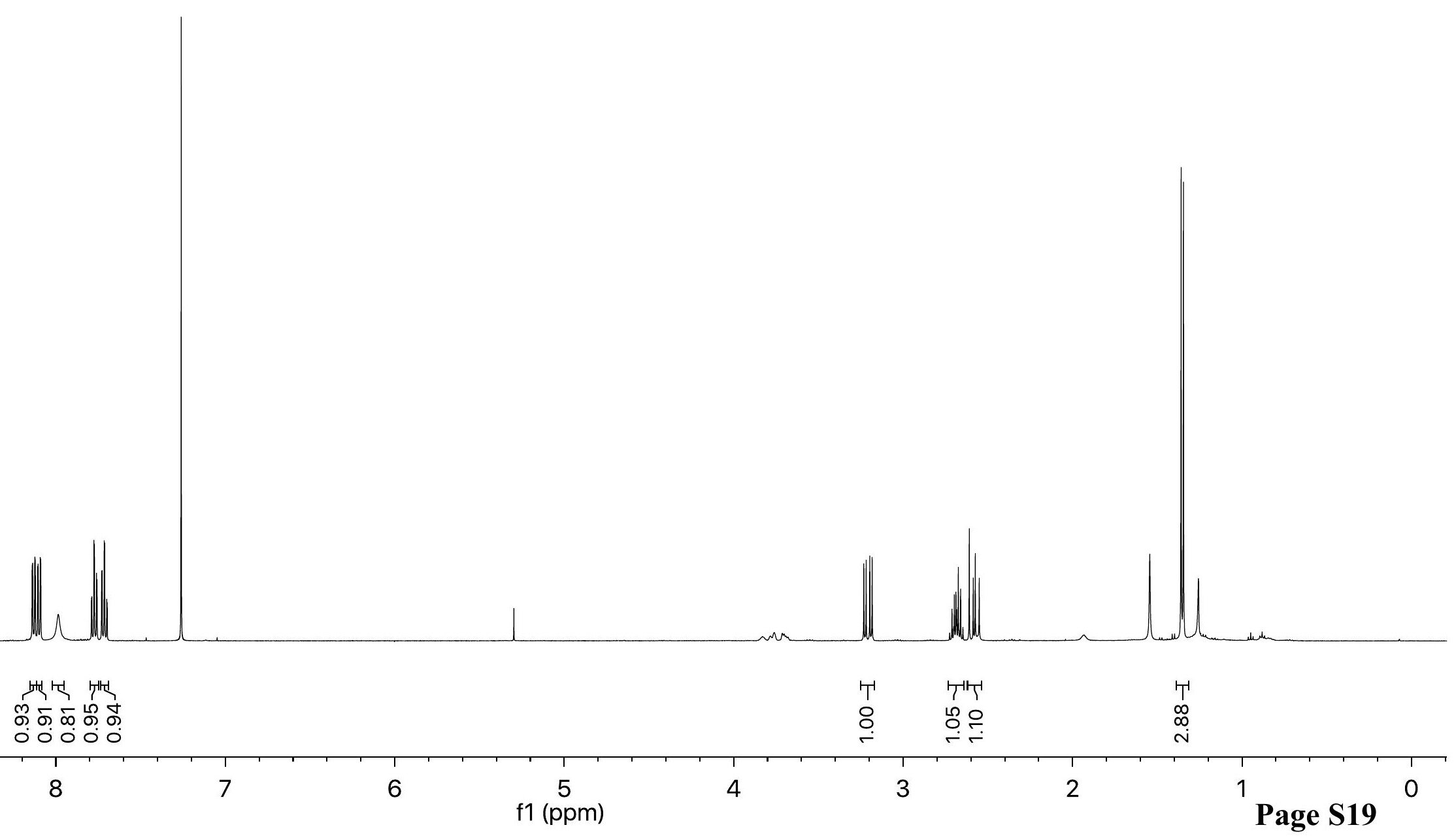




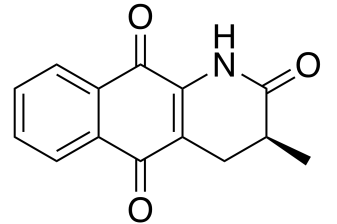

11, ${ }^{13} \mathrm{C}$ NMR $\left(126 \mathrm{MHz}, \mathrm{CDCl}_{3}\right)$ 


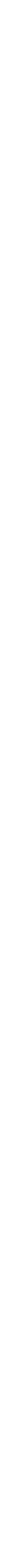


<smiles>Cc1cc2c([nH]c1=O)C(=O)c1ccccc1C2=O</smiles>

12, ${ }^{13} \mathrm{C} \mathrm{NMR}\left(126 \mathrm{MHz}, \mathrm{CDCl}_{3}\right)$
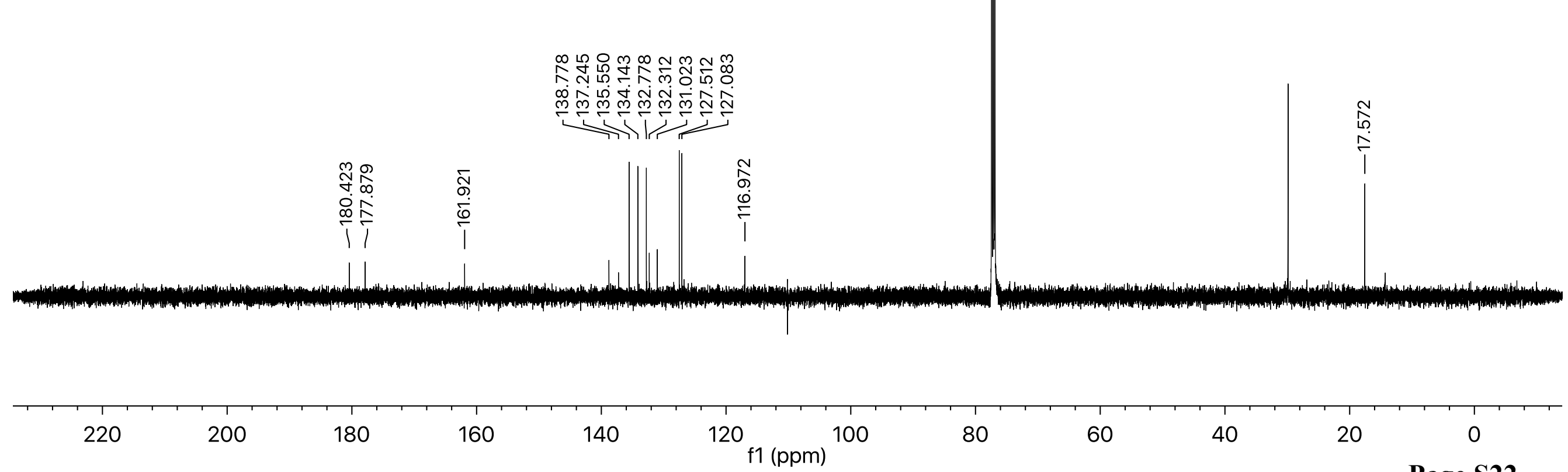

Page S22 


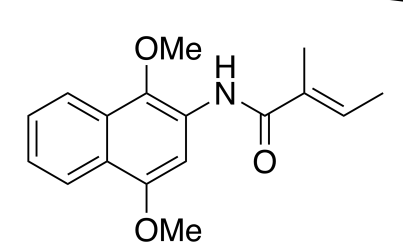

S4, ${ }^{1} \mathrm{H}$ NMR $\left(500 \mathrm{MHz}, \mathrm{CDCl}_{3}\right)$

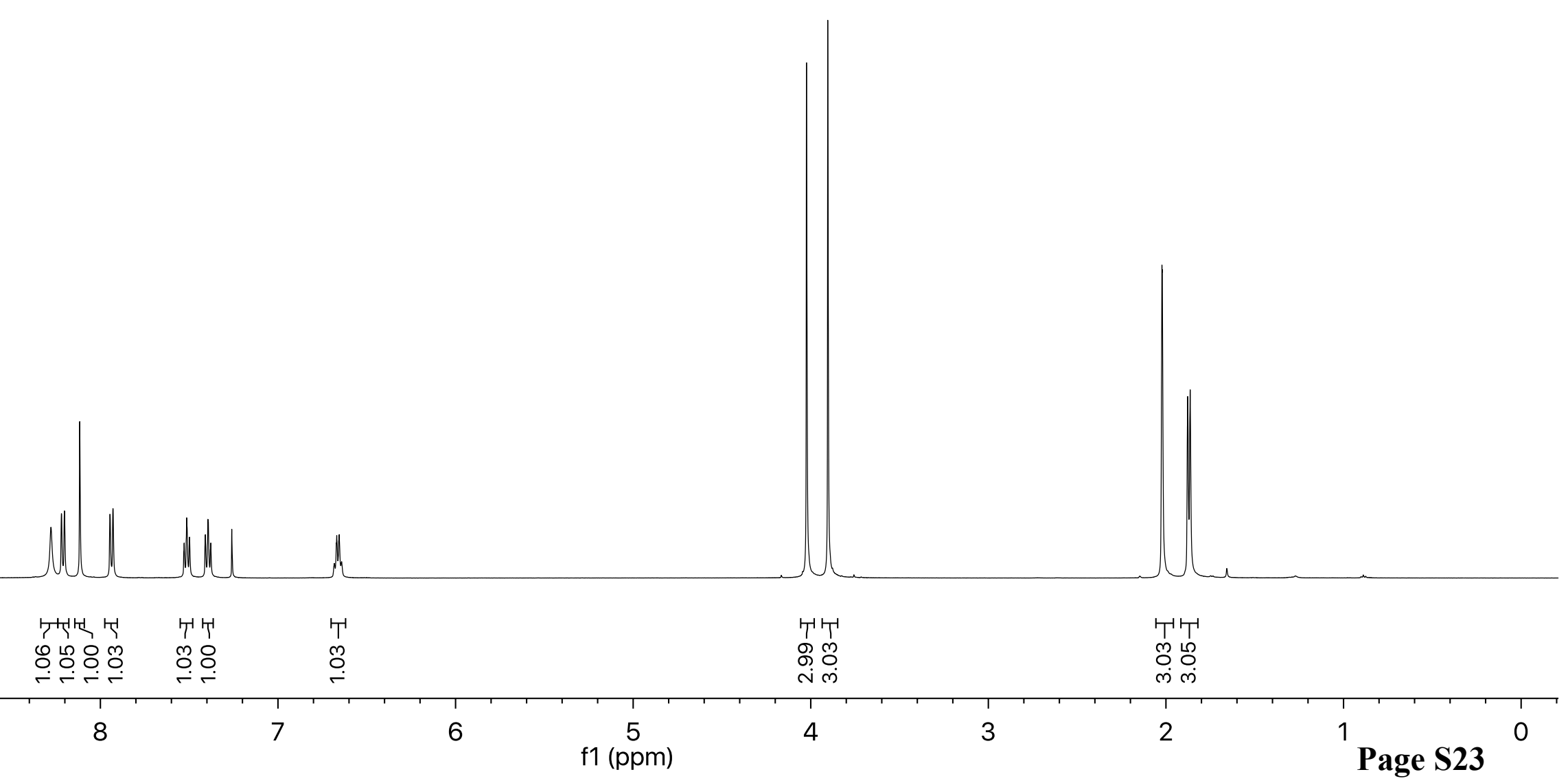




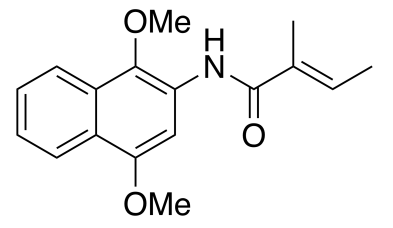

S4, ${ }^{13} \mathrm{C} \mathrm{NMR}\left(126 \mathrm{MHz}, \mathrm{CDCl}_{3}\right)$

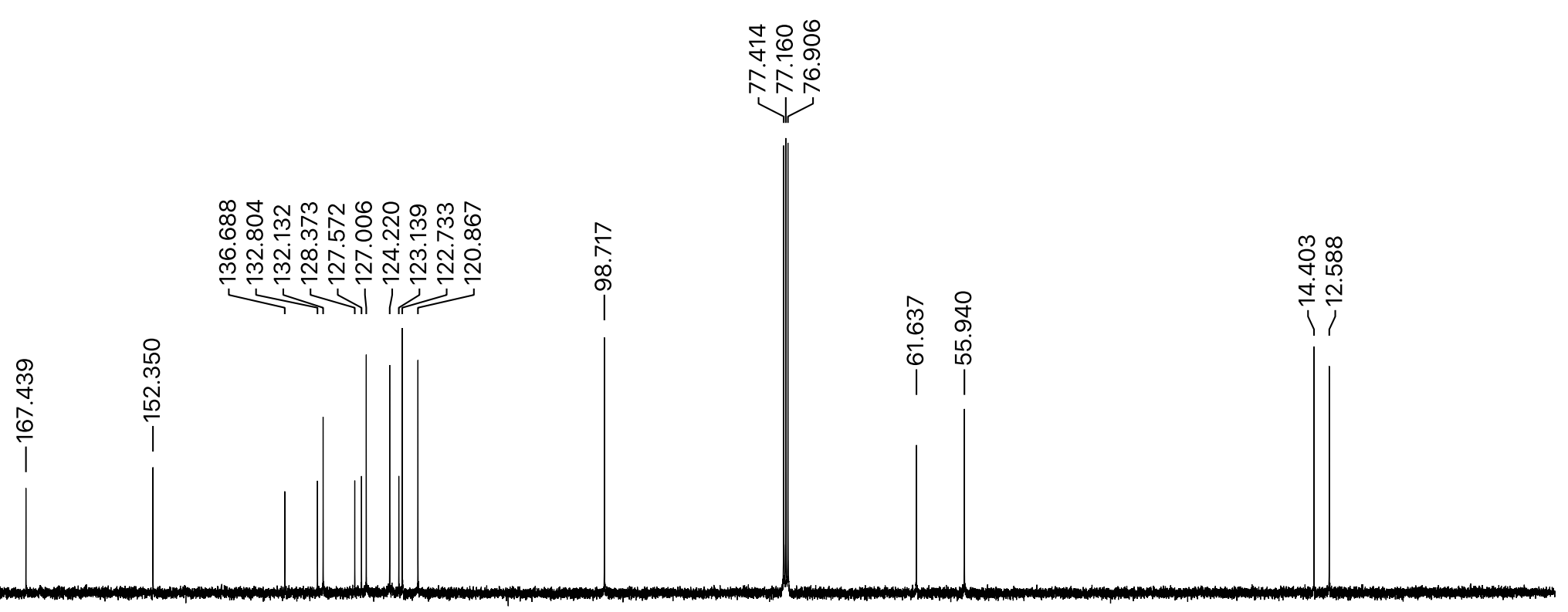

0




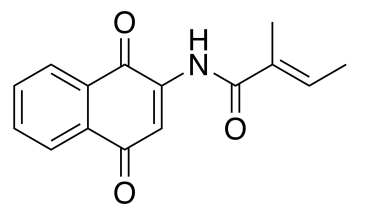

13, $\left.{ }^{13} \mathrm{C} \mathrm{NMR} \mathrm{(126} \mathrm{MHz,} \mathrm{CDCl}_{3}\right)$

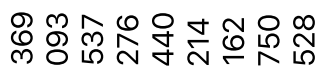

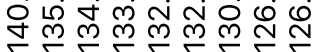

ता।

ㅎํㅇㅇㅇㅇ

송

诸

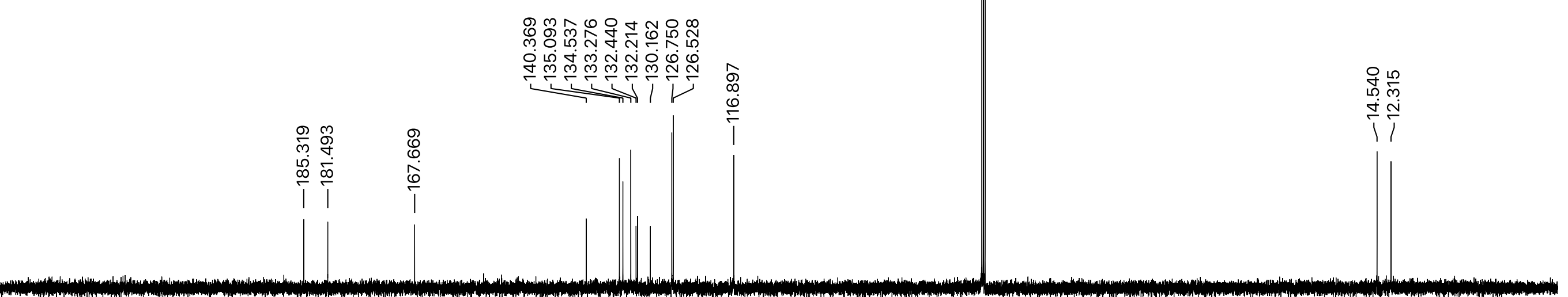

ㅇํㅇำ

$\stackrel{+}{i}$

\begin{tabular}{|c|c|c|c|c|c|c|c|c|c|c|c|}
\hline 220 & 200 & 180 & 160 & 140 & 120 & 100 & 80 & 60 & 40 & 20 & Page S26 \\
\hline
\end{tabular}




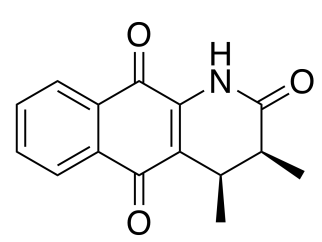

14, ${ }^{1} \mathrm{H} \mathrm{NMR}\left(500 \mathrm{MHz}, \mathrm{CDCl}_{3}\right)$

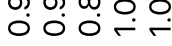




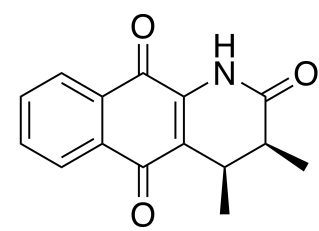

14, $\left.{ }^{13} \mathrm{C} \mathrm{NMR} \mathrm{(126} \mathrm{MHz,} \mathrm{CDCl}_{3}\right)$

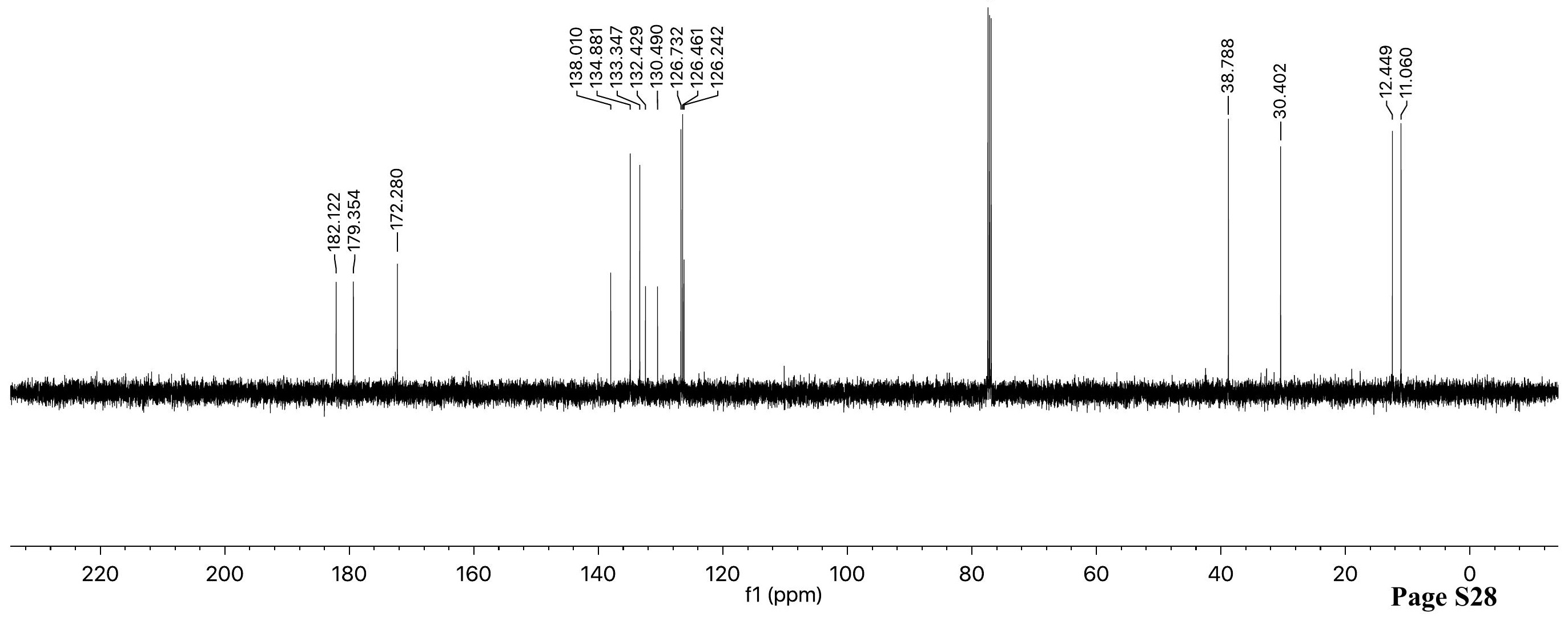




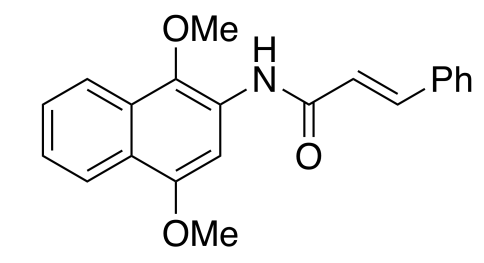

S5, ${ }^{1} \mathrm{H}$ NMR $\left(500 \mathrm{MHz}, \mathrm{CDCl}_{3}\right)$

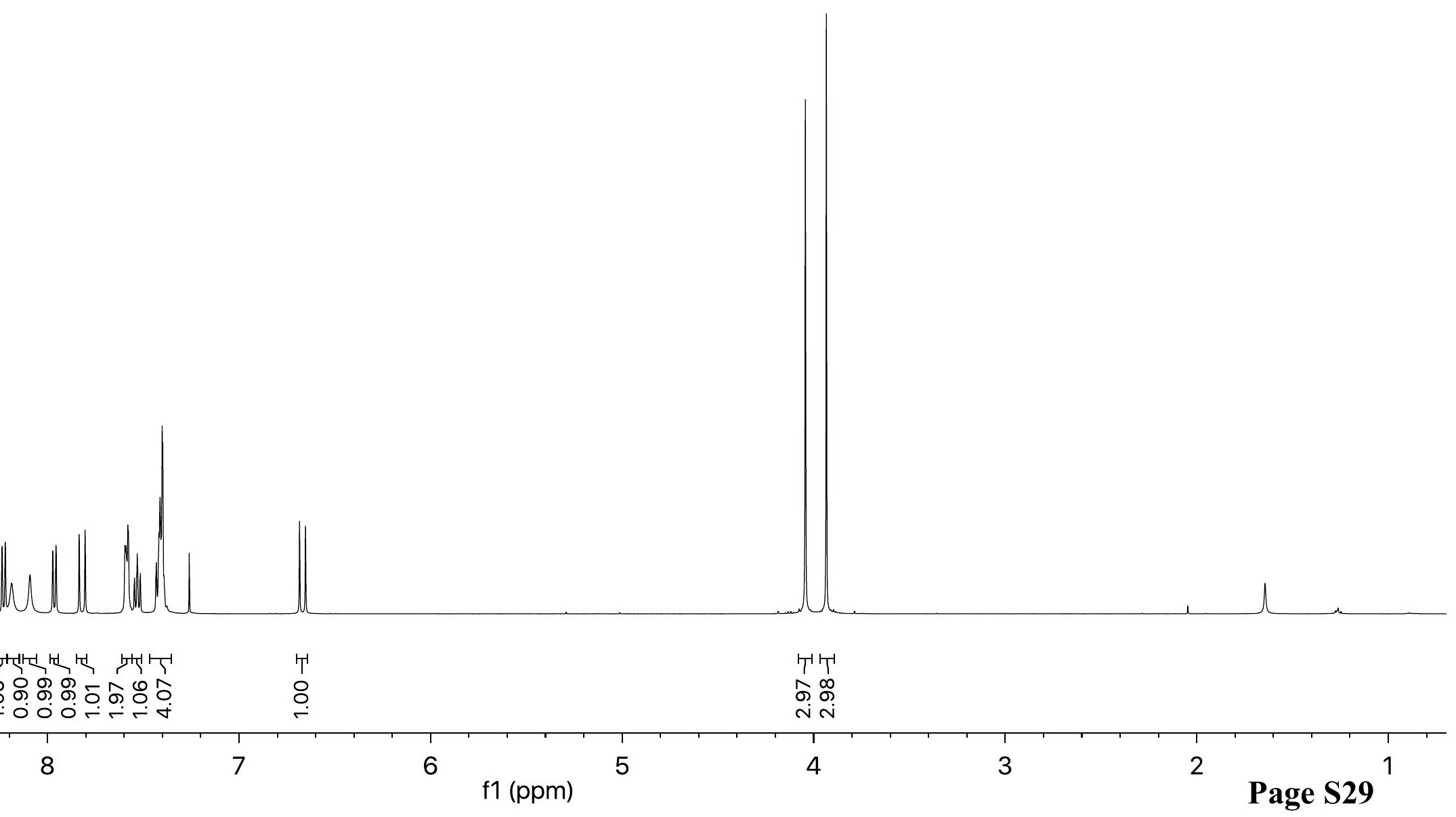




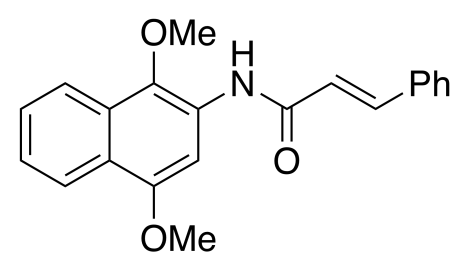

S5, $\left.{ }^{13} \mathrm{C} \mathrm{NMR} \mathrm{(126} \mathrm{MHz,} \mathrm{CDCl}_{3}\right)$

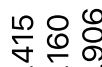

穴里

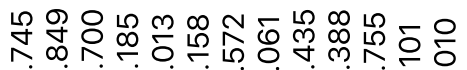

ฟั

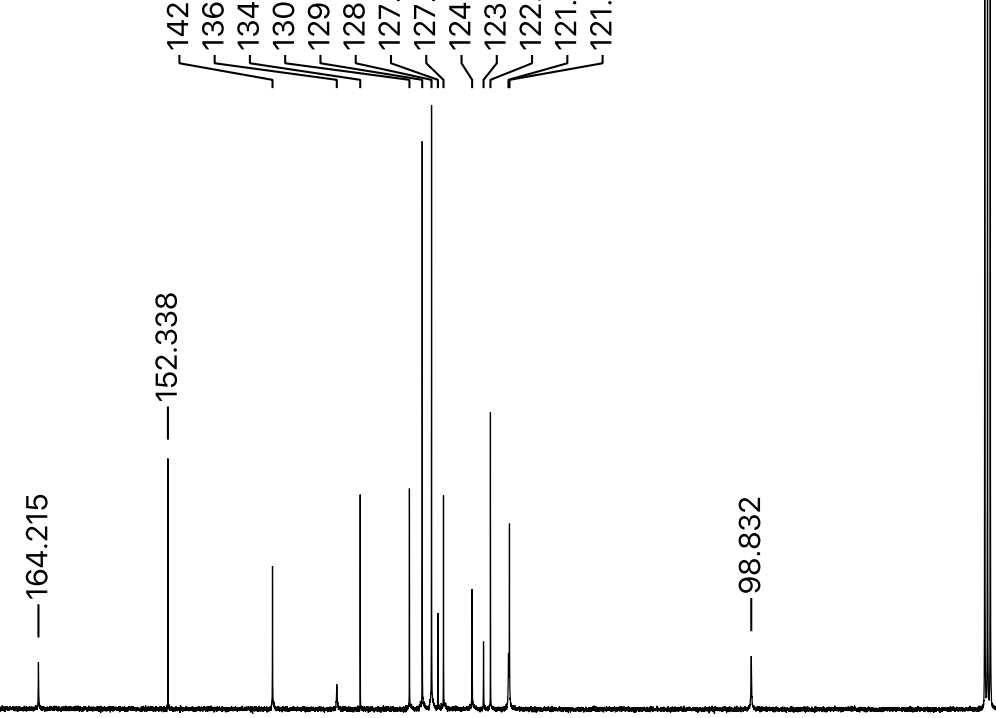

$\stackrel{\circ}{\infty}$

$\stackrel{\infty}{\infty} \stackrel{0}{\circ}$

ธं

|

\begin{tabular}{llllllllll}
\hline 20 & 200 & 180 & 160 & 140 & 120 & 100 & 80 & 60 & 40
\end{tabular}




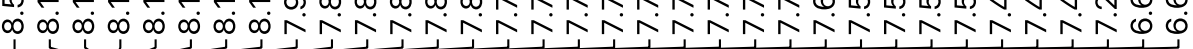

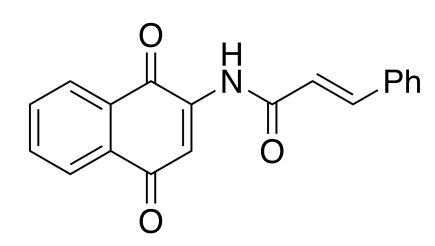

16, ${ }^{1} \mathrm{H} \mathrm{NMR}\left(500 \mathrm{MHz}, \mathrm{CDCl}_{3}\right)$

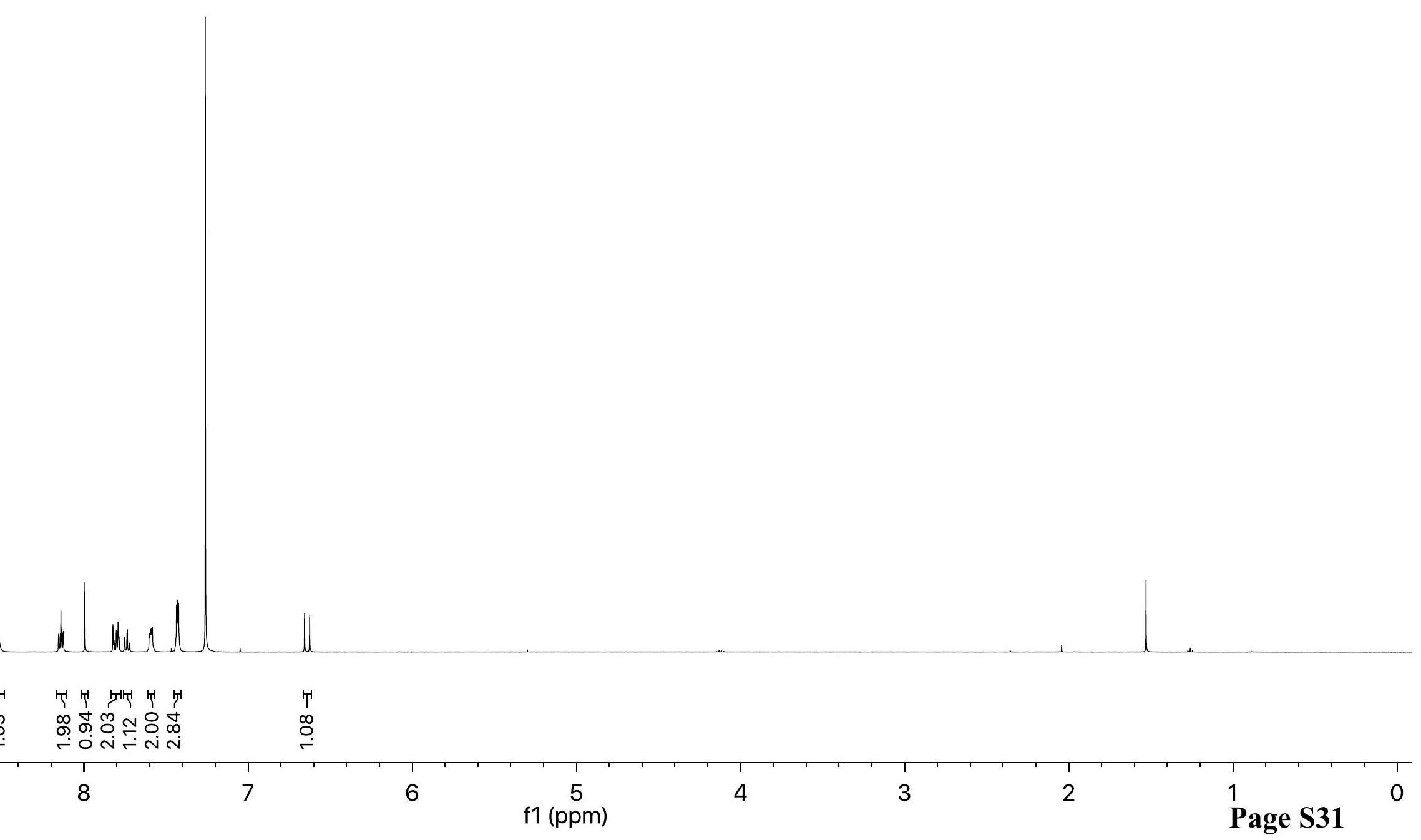




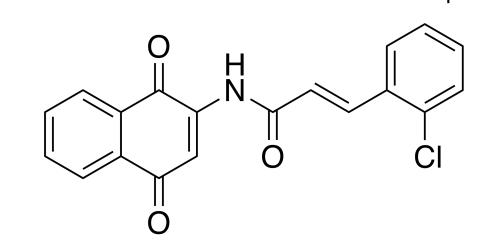

19, ${ }^{1} \mathrm{H}$ NMR (500 MHz, $\mathrm{CDCl}_{3}$ ) 


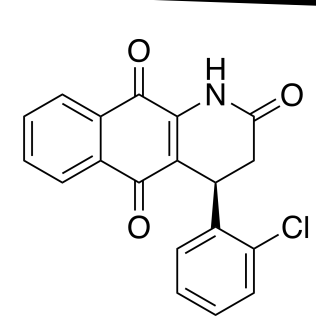

20, ${ }^{1} \mathrm{H}$ NMR $\left(400 \mathrm{MHz}, \mathrm{CDCl}_{3}\right)$

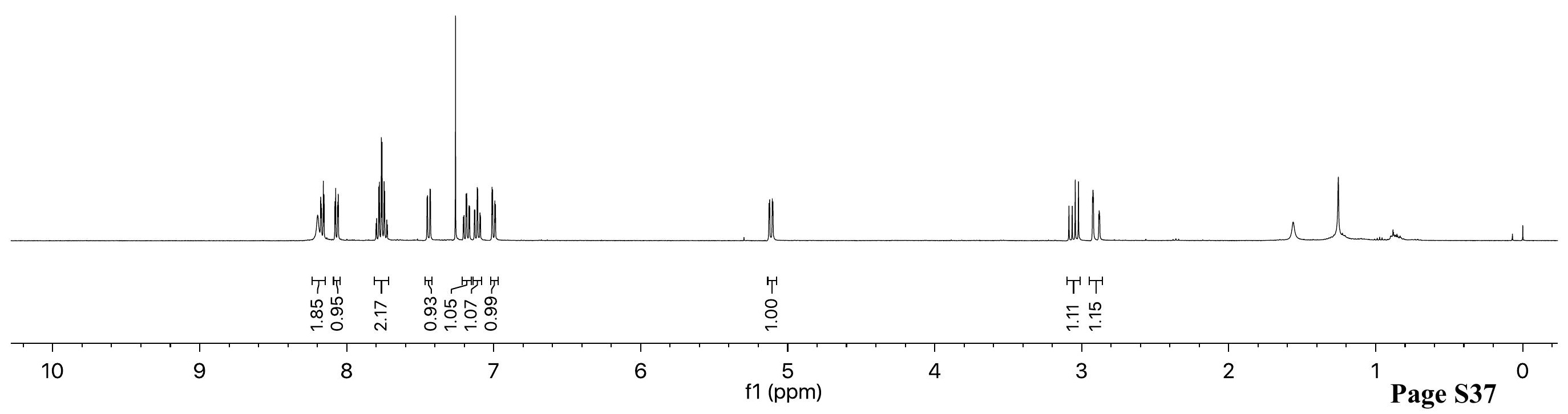




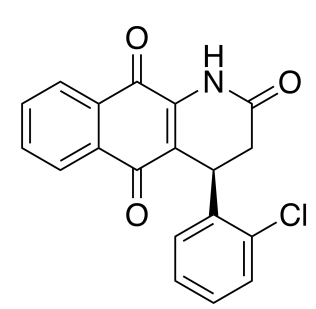

20, ${ }^{13} \mathrm{C}$ NMR (126 MHz, $\mathrm{CDCl}_{3}$ )

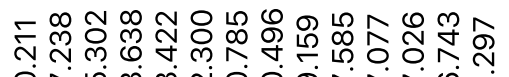
ợ

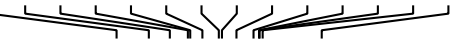




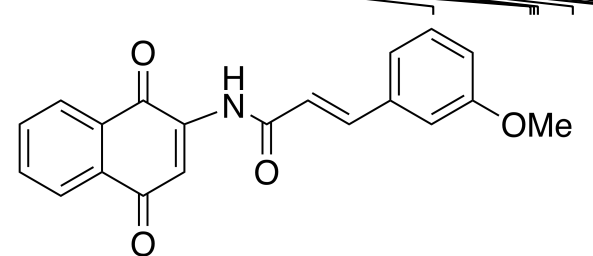

22, ${ }^{1} \mathrm{H}$ NMR (500 MHz, $\mathrm{CDCl}_{3}$ )

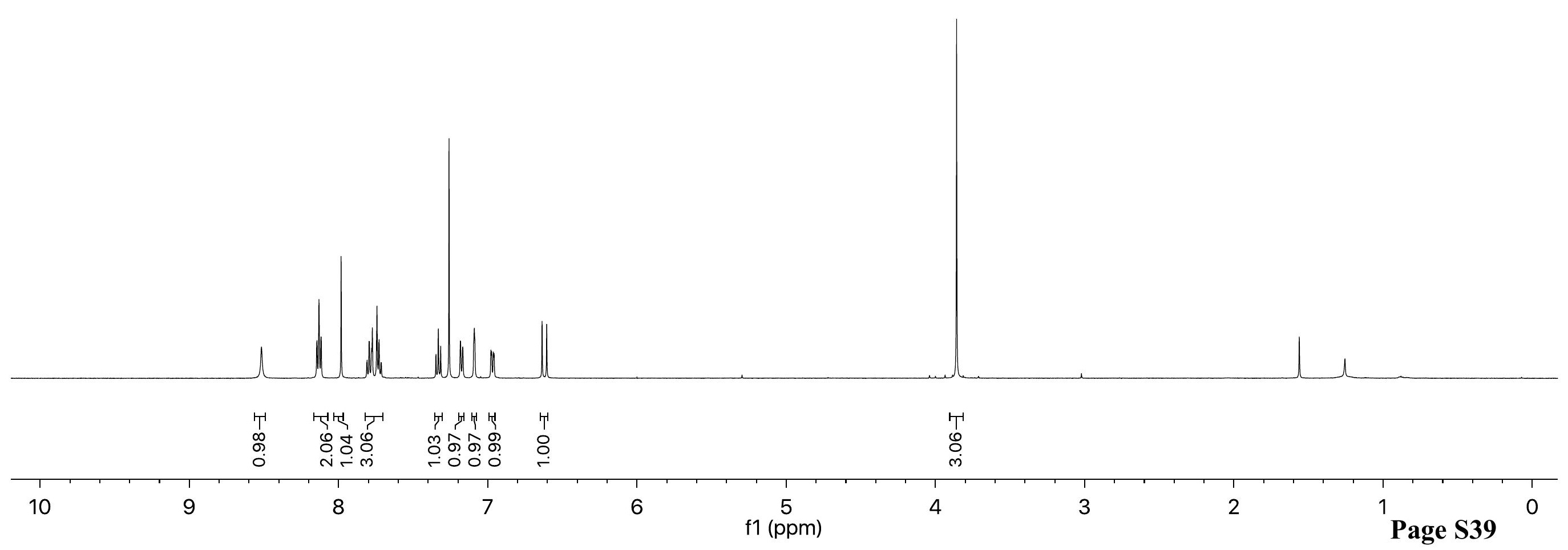




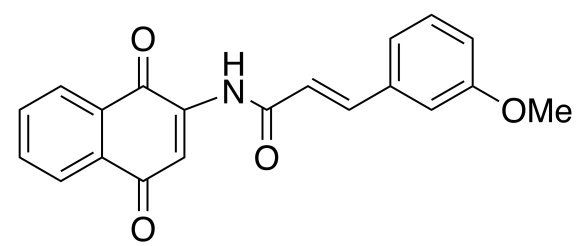

22, $\left.{ }^{13} \mathrm{C} \mathrm{NMR} \mathrm{(126} \mathrm{MHz,} \mathrm{CDCl}_{3}\right)$

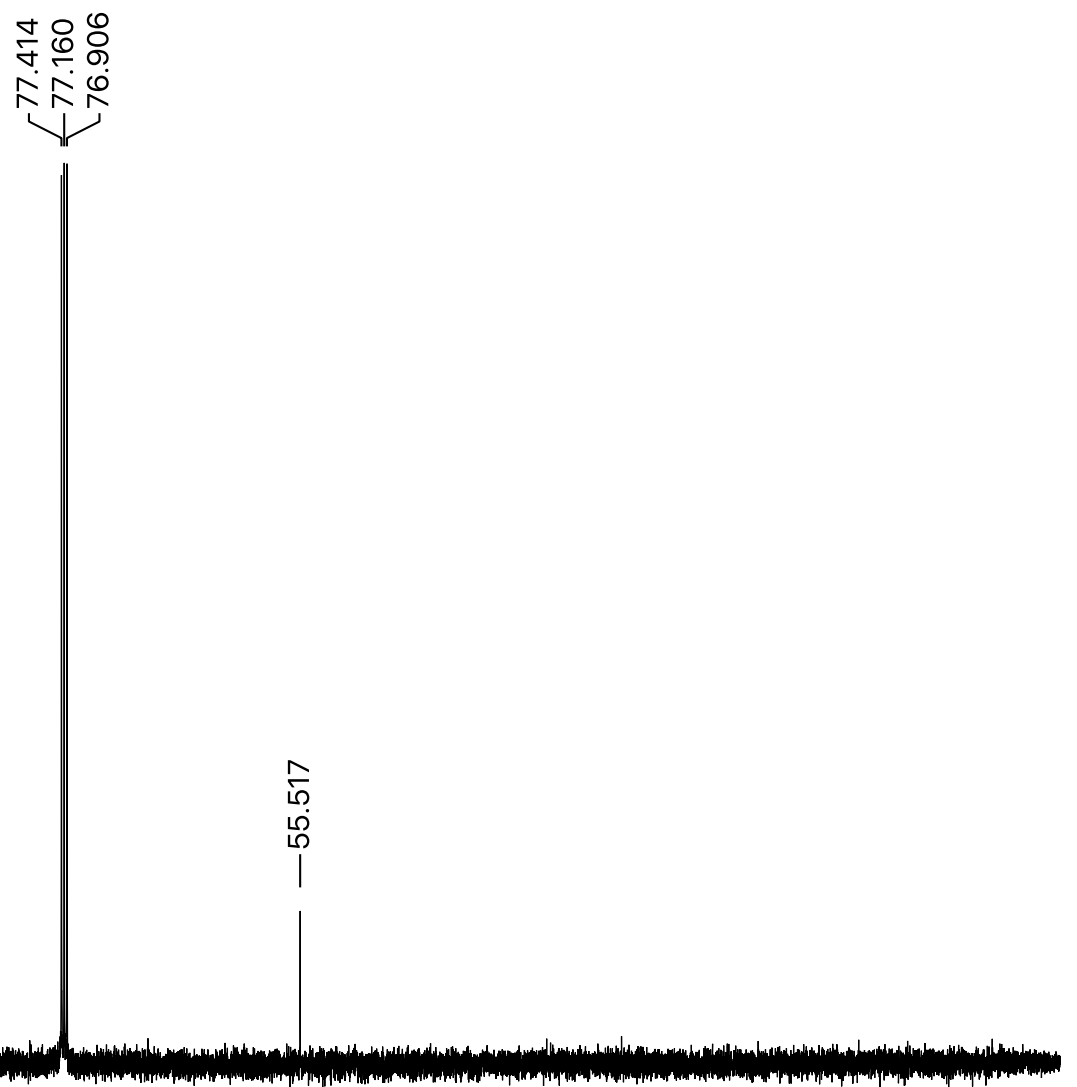

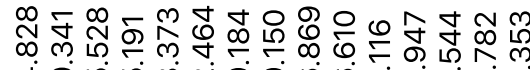

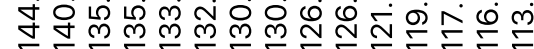

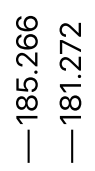

ํำ

ஸ் ญ்

$\overbrace{}^{\infty}$

-

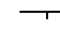

$100 \quad 80$

80

60

40

20

Page S40 


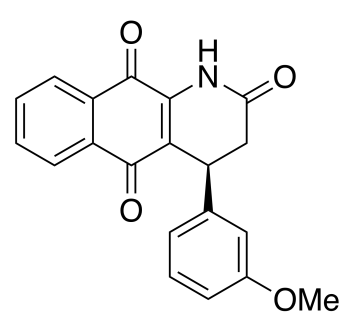

23, ${ }^{1} \mathrm{H}$ NMR $\left(500 \mathrm{MHz}, \mathrm{CDCl}_{3}\right)$

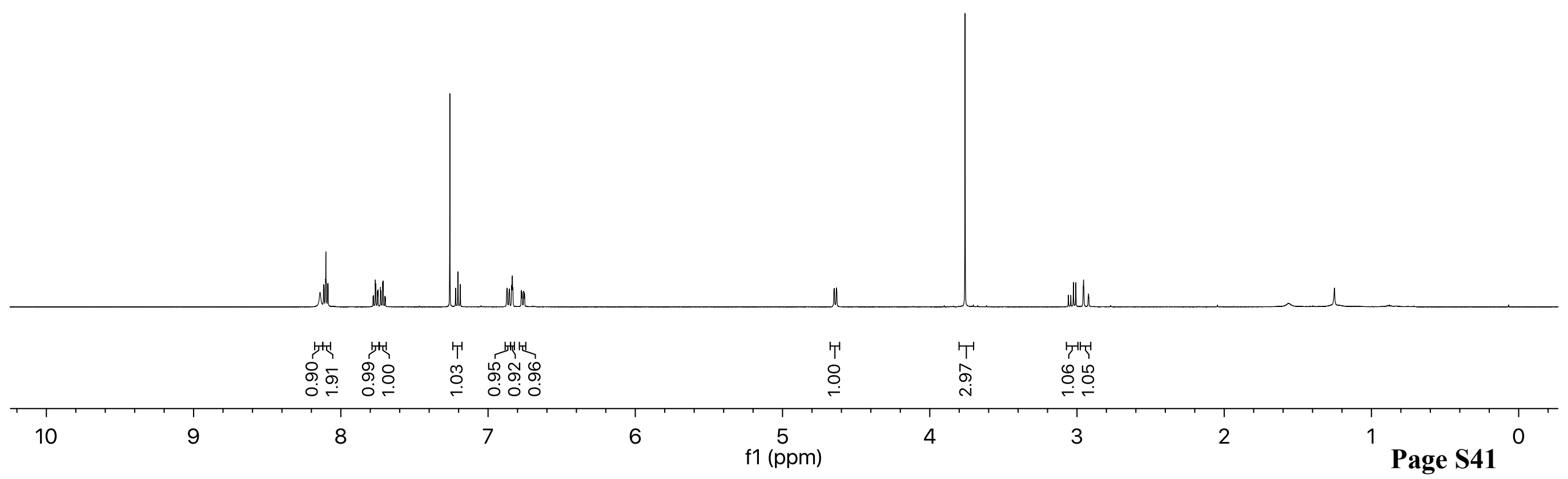




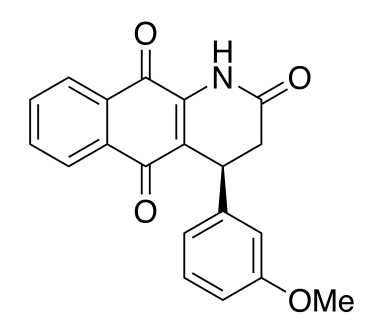

23, $\left.{ }^{13} \mathrm{C} \mathrm{NMR} \mathrm{(126} \mathrm{MHz,} \mathrm{CDCl}_{3}\right)$

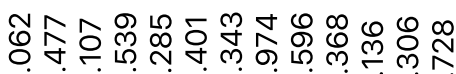

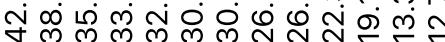

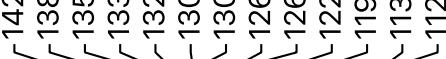

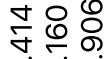

Кำ

VI

1)
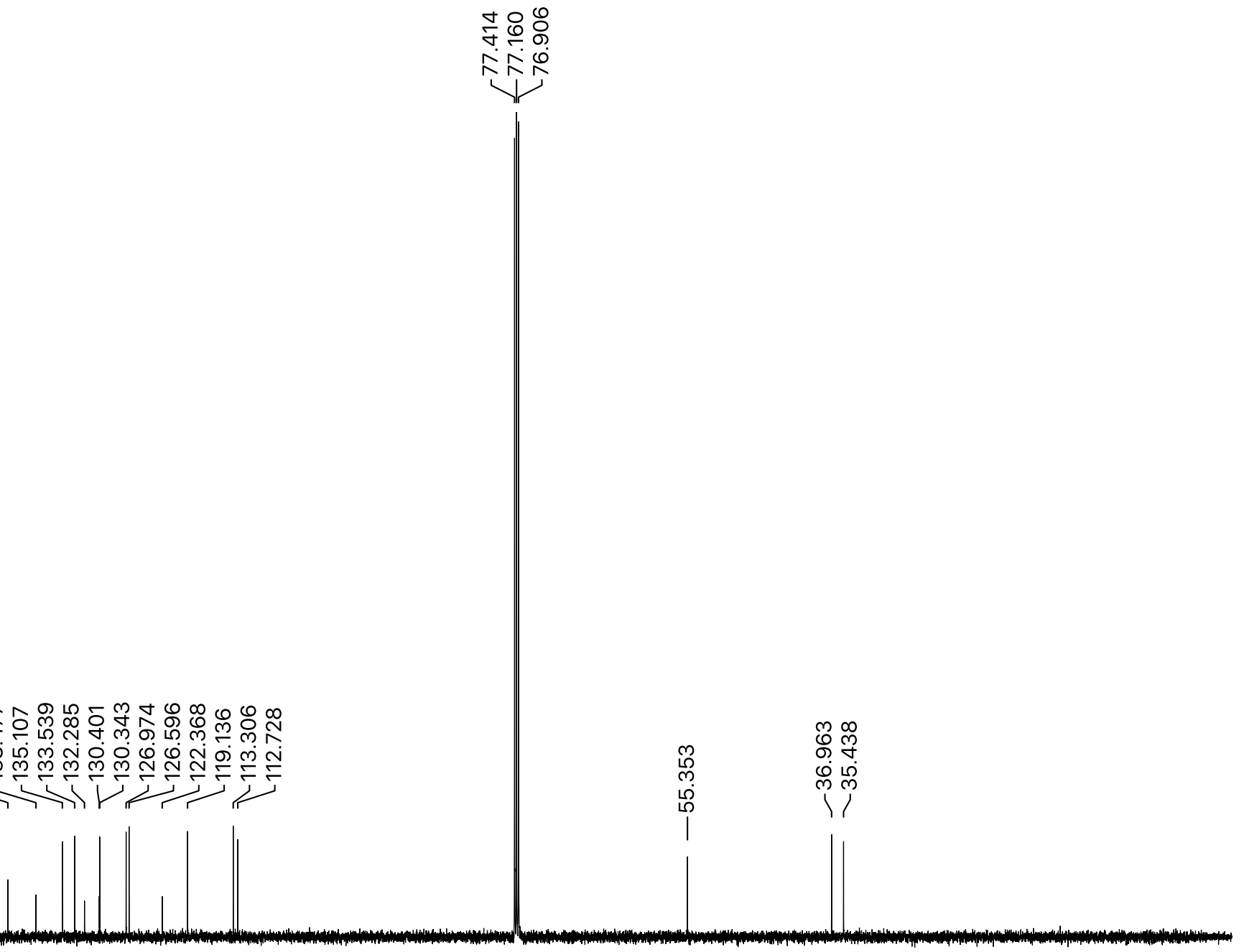

$\begin{array}{llllllllll} & 220 & 180 & 160 & 140 & 120 & 100 & 80 & 60 & 40\end{array}$




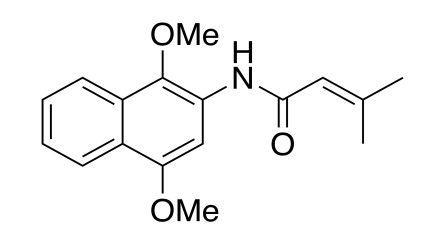

S6, ${ }^{1} \mathrm{H}$ NMR $\left(500 \mathrm{MHz}, \mathrm{CDCl}_{3}\right)$

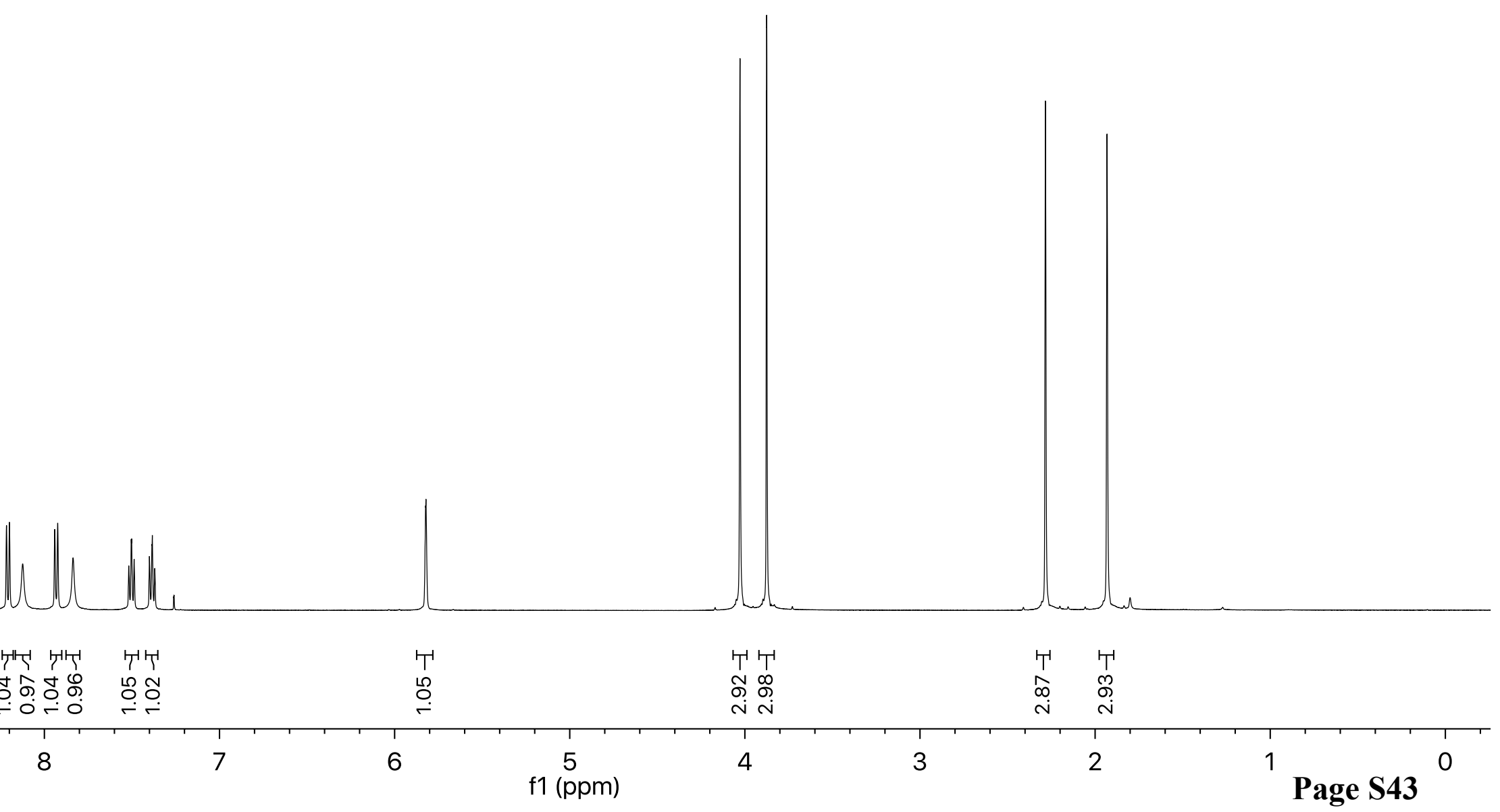




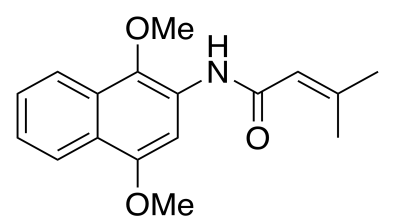

S6, ${ }^{13} \mathrm{C}$ NMR $\left(126 \mathrm{MHz}, \mathrm{CDCl}_{3}\right)$

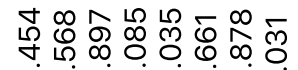

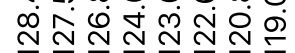

눈

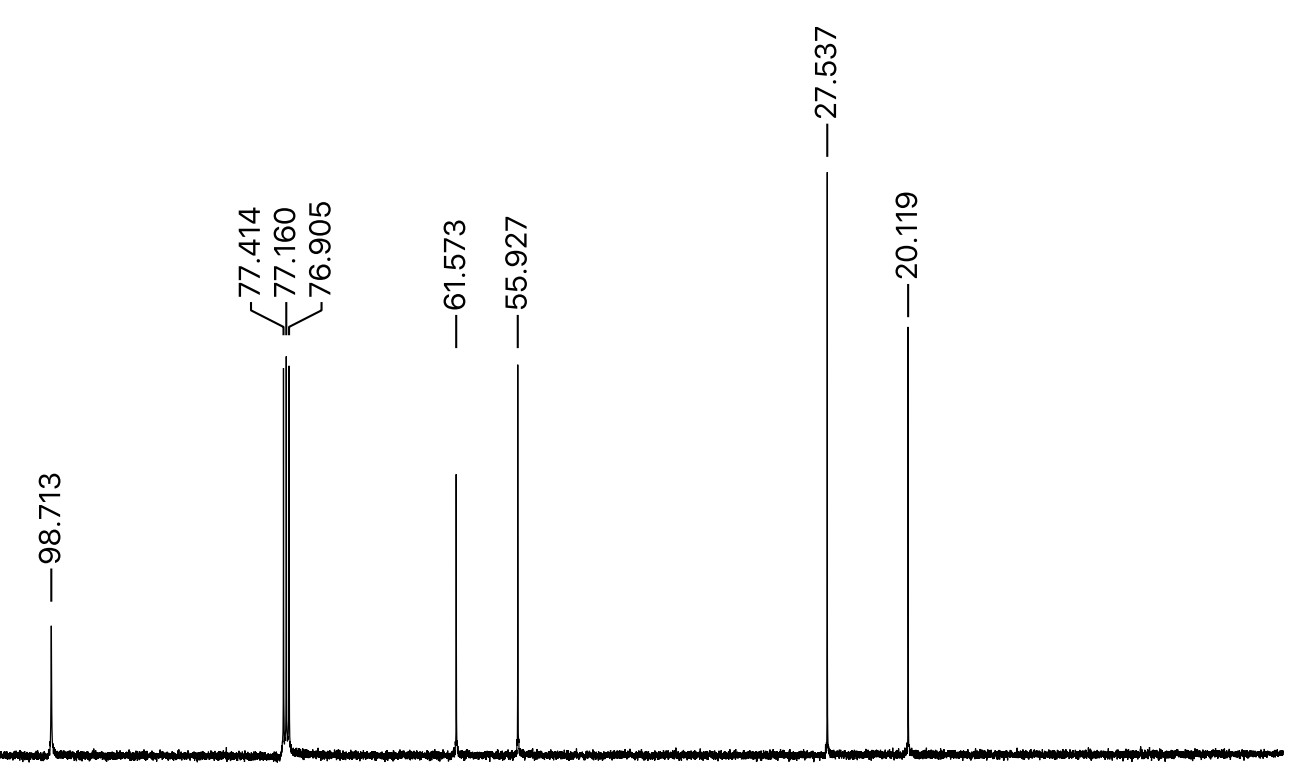




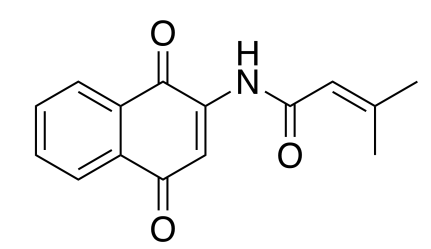

29, ${ }^{1} \mathrm{H} \mathrm{NMR}\left(500 \mathrm{MHz}, \mathrm{CDCl}_{3}\right)$

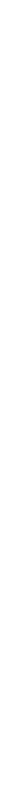




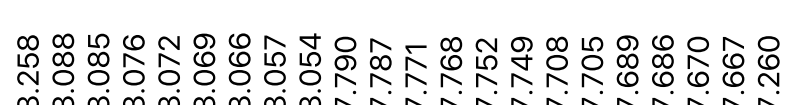

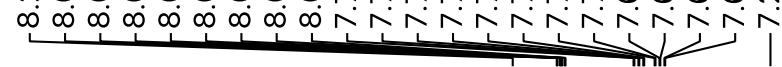

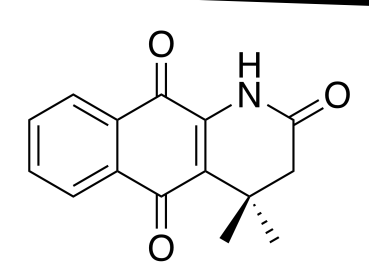

30, ${ }^{1} \mathrm{H} \mathrm{NMR}\left(500 \mathrm{MHz}, \mathrm{CDCl}_{3}\right)$

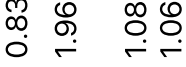




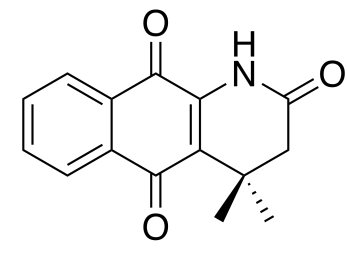

30, $\left.{ }^{13} \mathrm{C} \mathrm{NMR} \mathrm{(126} \mathrm{MHz,} \mathrm{CDCl}_{3}\right)$

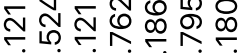

m pm m户 miñ
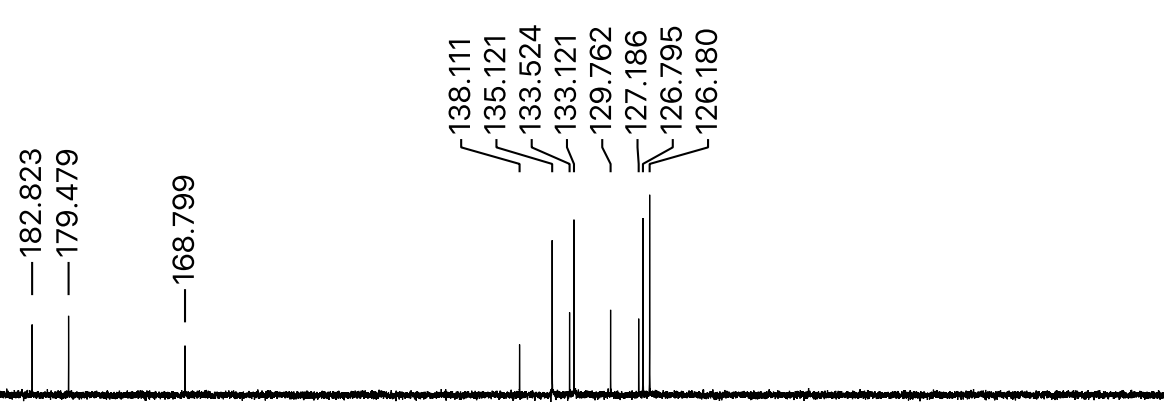

늠

Nํำ
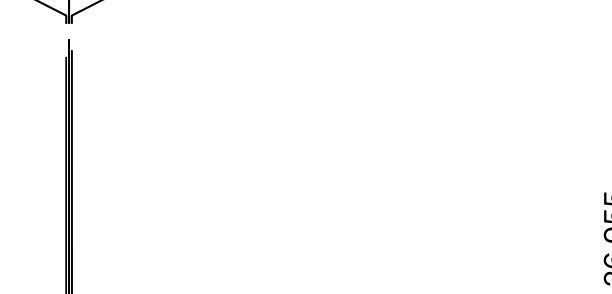


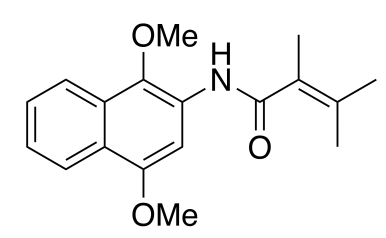

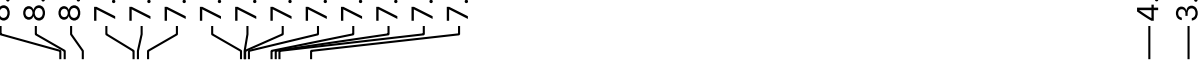

S7, ${ }^{1} \mathrm{H}$ NMR $\left(500 \mathrm{MHz}, \mathrm{CDCl}_{3}\right)$

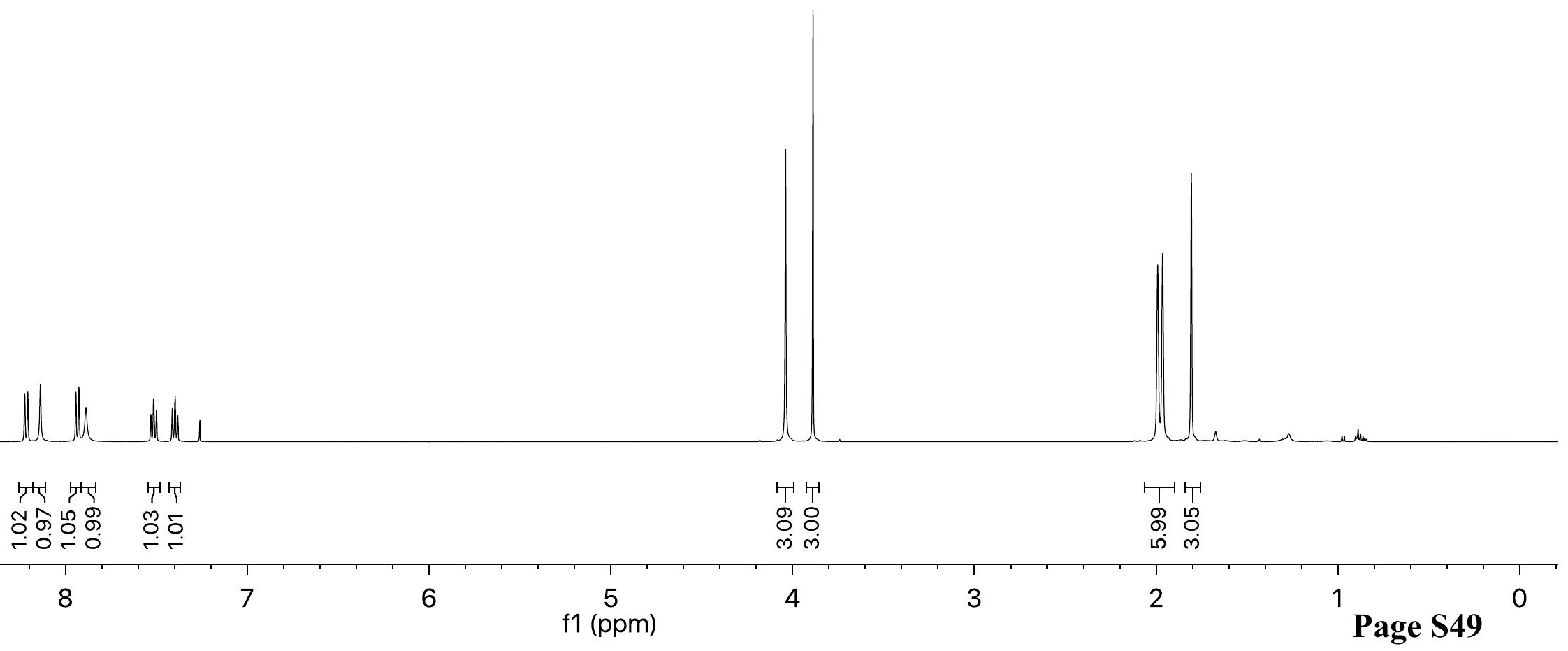




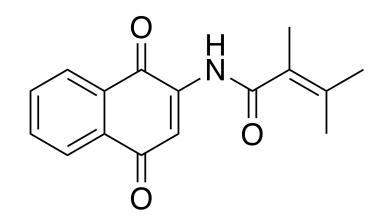

31, ${ }^{1} \mathrm{H}$ NMR (500 MHz, $\left.\mathrm{CDCl}_{3}\right)$

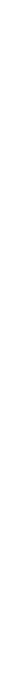




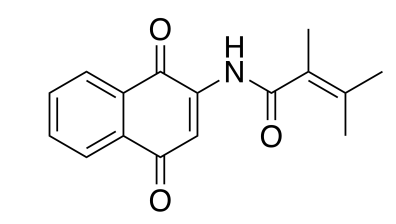

31, ${ }^{13} \mathrm{C}$ NMR $\left(126 \mathrm{MHz}, \mathrm{CDCl}_{3}\right)$

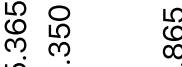

i)

।

$220 \quad 200$




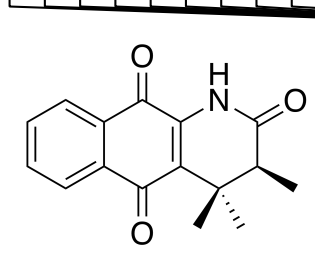

32, ${ }^{1} \mathrm{H}$ NMR $\left(500 \mathrm{MHz}, \mathrm{CDCl}_{3}\right)$

化 计 ㅅ

$$
10
$$




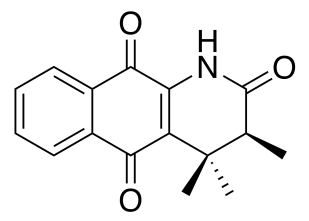

32, $\left.{ }^{13} \mathrm{C} \mathrm{NMR} \mathrm{(126} \mathrm{MHz,} \mathrm{CDCl}_{3}\right)$

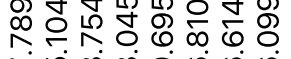

ণิ

m

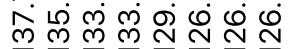

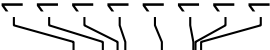

효

손 


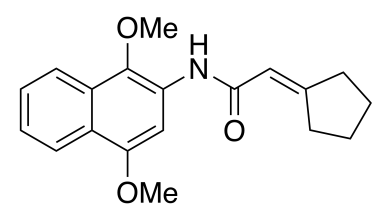

S8, ${ }^{1} \mathrm{H}$ NMR $\left(500 \mathrm{MHz}, \mathrm{CDCl}_{3}\right)$

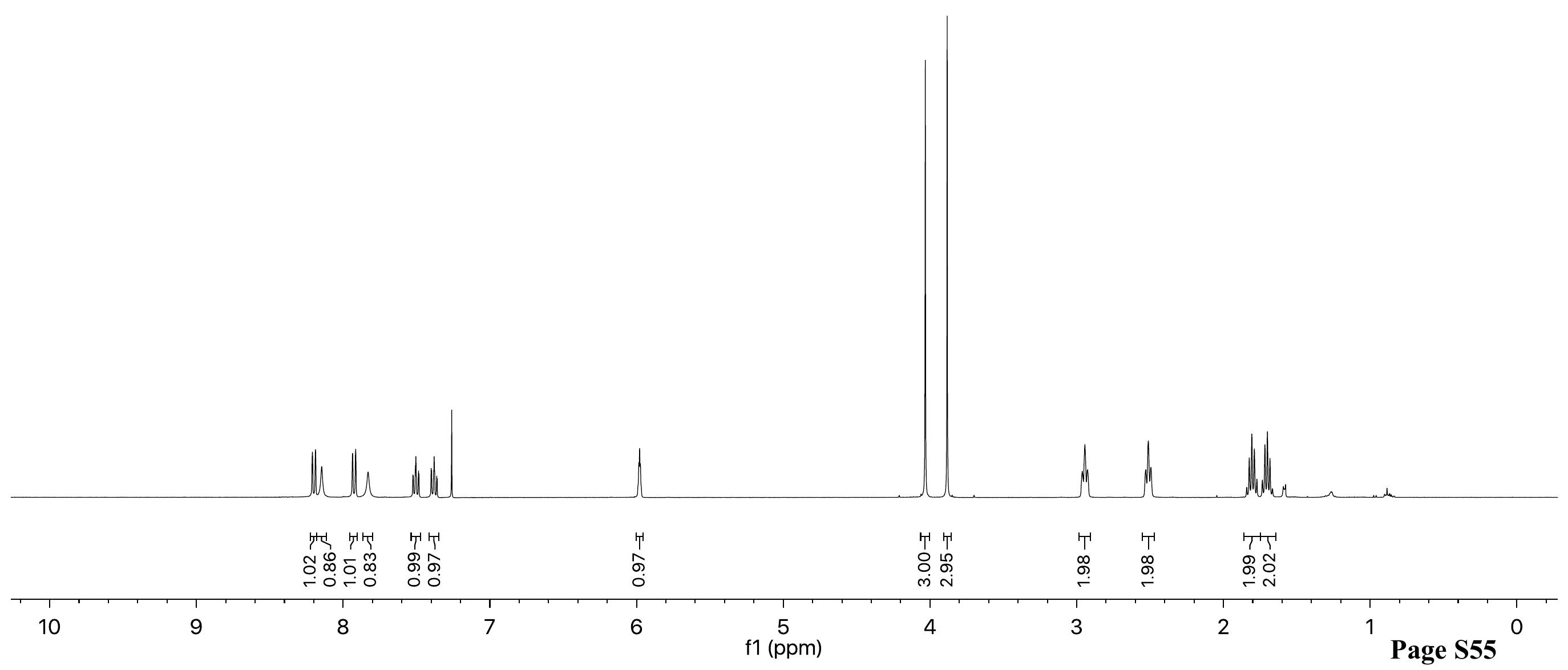




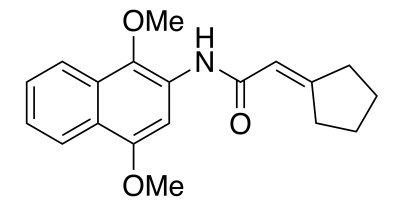

S8, $\left.{ }^{13} \mathrm{C} \mathrm{NMR} \mathrm{(126} \mathrm{MHz,} \mathrm{CDCl}_{3}\right)$

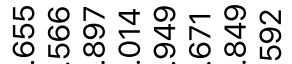

茫

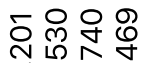

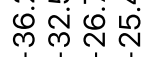
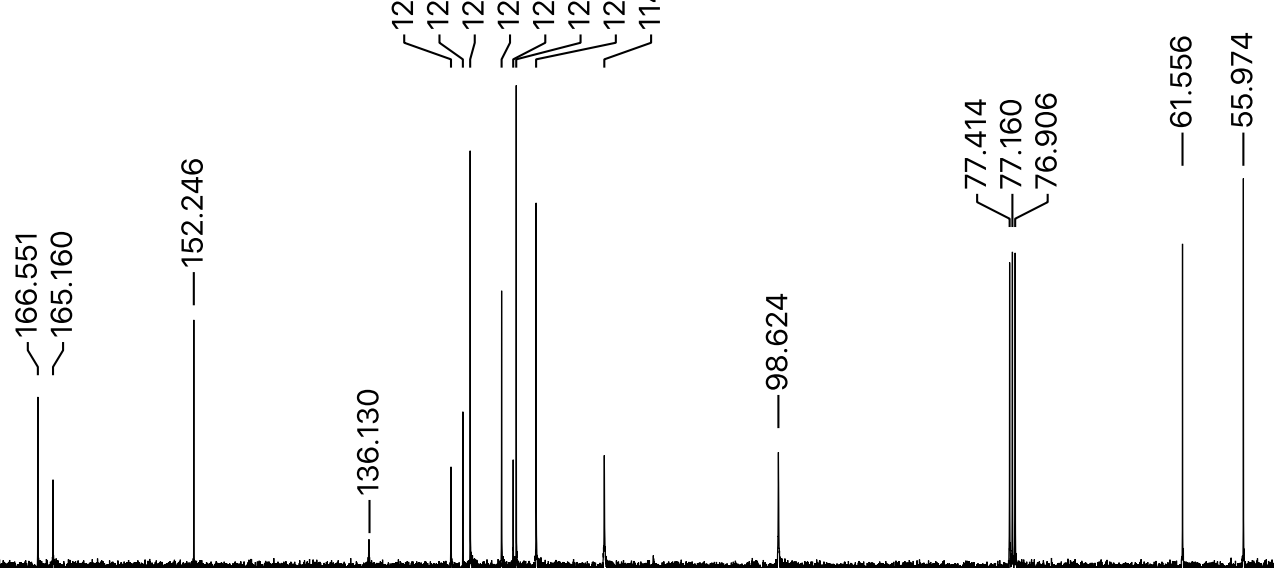

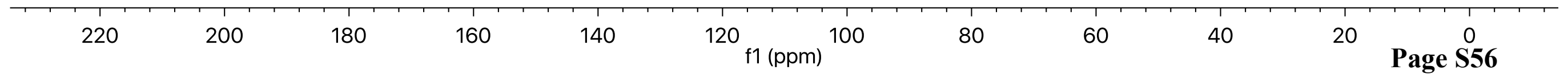




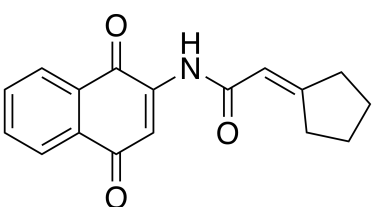

33, ${ }^{1} \mathrm{H}$ NMR $\left(500 \mathrm{MHz}, \mathrm{CDCl}_{3}\right)$

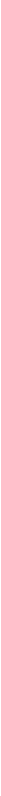




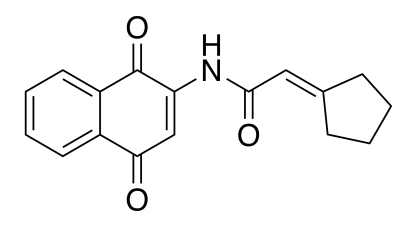

33, ${ }^{13} \mathrm{C}$ NMR $\left(126 \mathrm{MHz}, \mathrm{CDCl}_{3}\right)$
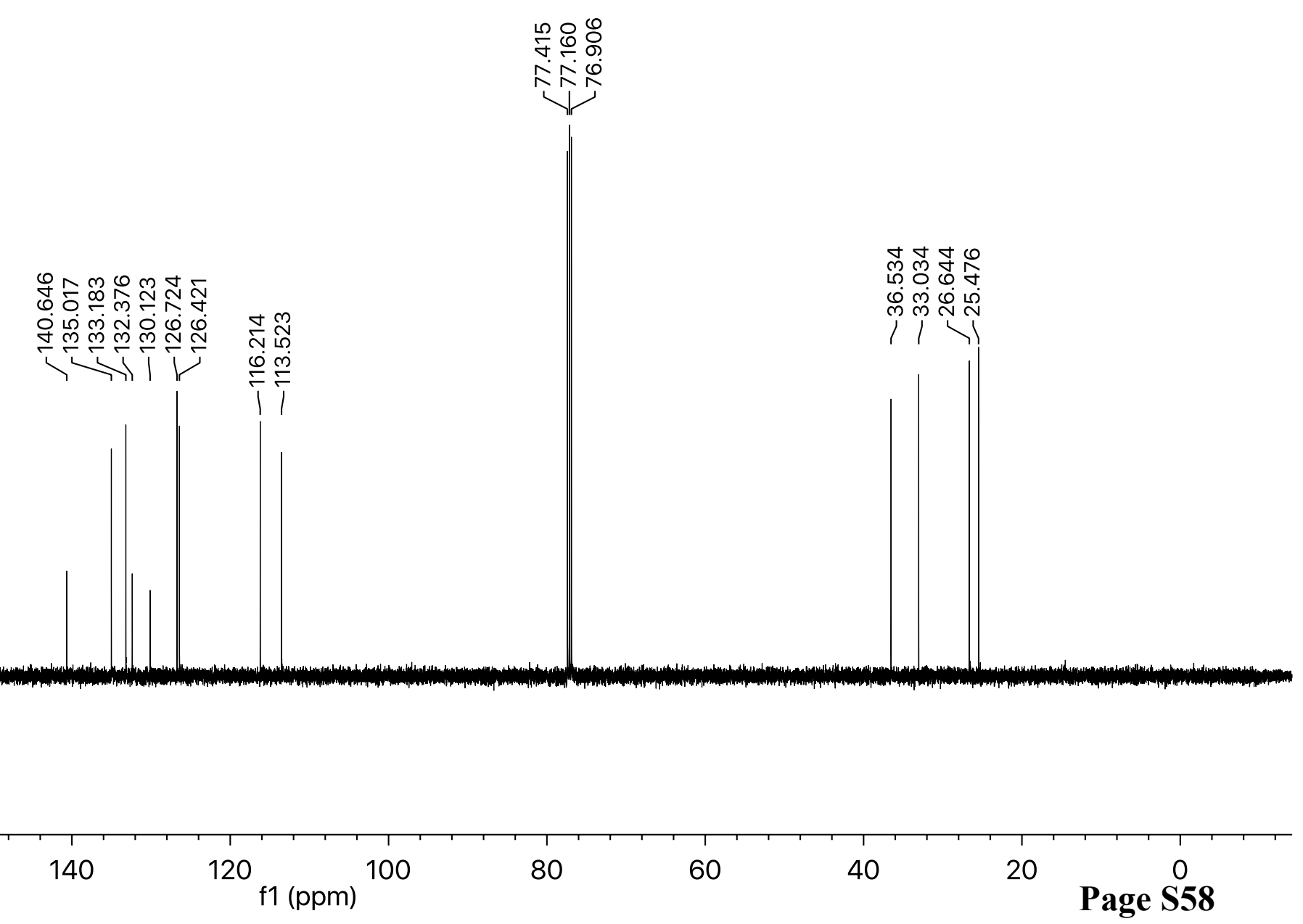

장

$\frac{\infty}{m}$

营

ம

ำ

| 1 


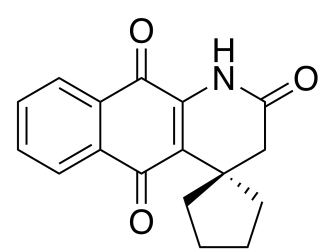

34, ${ }^{1} \mathrm{H}$ NMR (500 MHz, $\left.\mathrm{CDCl}_{3}\right)$

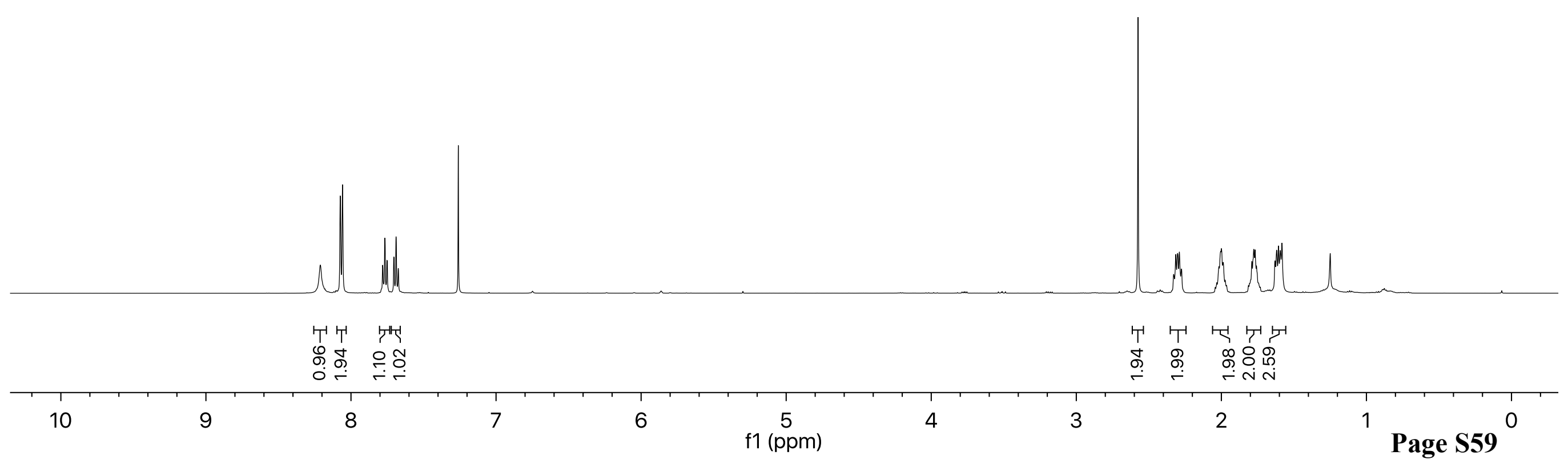




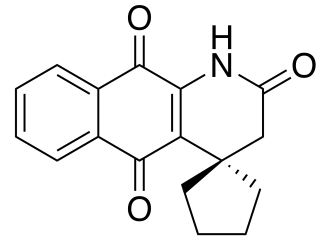

34, ${ }^{13} \mathrm{C}$ NMR (126 MHz, $\mathrm{CDCl}_{3}$ )

80




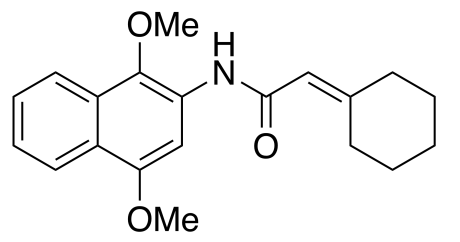

S9, ${ }^{13} \mathrm{C} \mathrm{NMR}\left(126 \mathrm{MHz}, \mathrm{CDCl}_{3}\right)$

\begin{tabular}{|c|c|c|c|c|c|c|c|c|c|c|c|}
\hline ' & 200 & 180 & 160 & 140 & 120 & 100 & 80 & 60 & 40 & 20 & $\begin{array}{c}0 \\
\text { Page } \\
\text { S62 }\end{array}$ \\
\hline
\end{tabular}




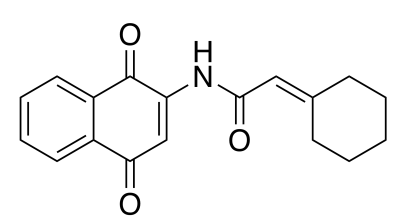

35, ${ }^{1} \mathrm{H}$ NMR $\left(500 \mathrm{MHz}, \mathrm{CDCl}_{3}\right)$

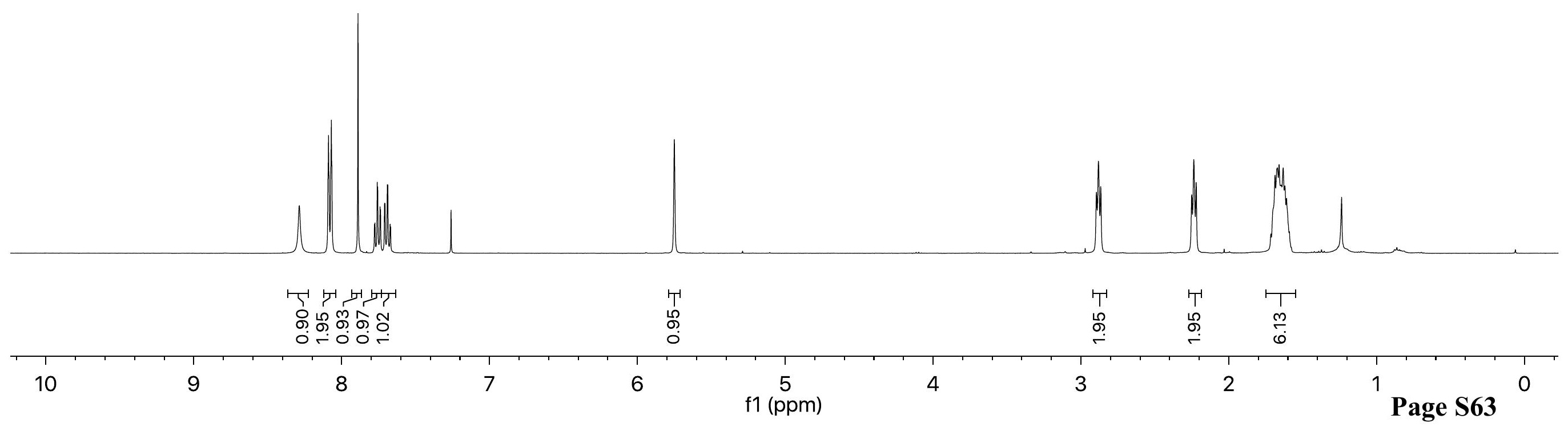




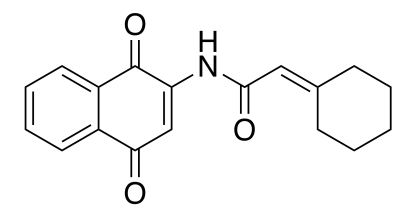

35, ${ }^{13} \mathrm{C}$ NMR (126 MHz, $\mathrm{CDCl}_{3}$ )

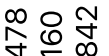 \\ 소용}

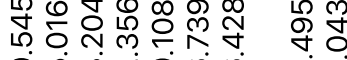

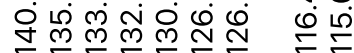

रोiा

용요

ம)

广

운홍

迸

\%

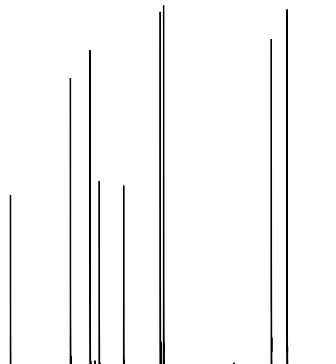

(in)

$$
-
$$




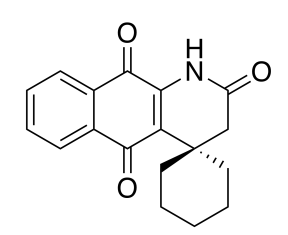

36, ${ }^{1} \mathrm{H}$ NMR $\left(500 \mathrm{MHz}, \mathrm{CDCl}_{3}\right)$

6. 


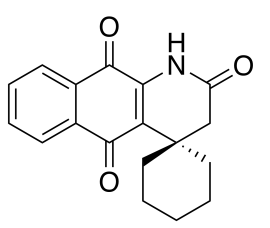

36, ${ }^{13} \mathrm{C} \mathrm{NMR}\left(126 \mathrm{MHz}, \mathrm{CDCl}_{3}\right)$

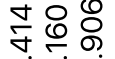

$\stackrel{\substack{\infty \\ \infty}}{\infty}$

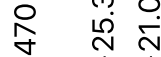

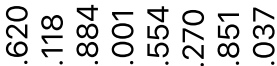

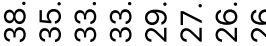

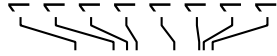

옹

๓ं

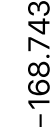
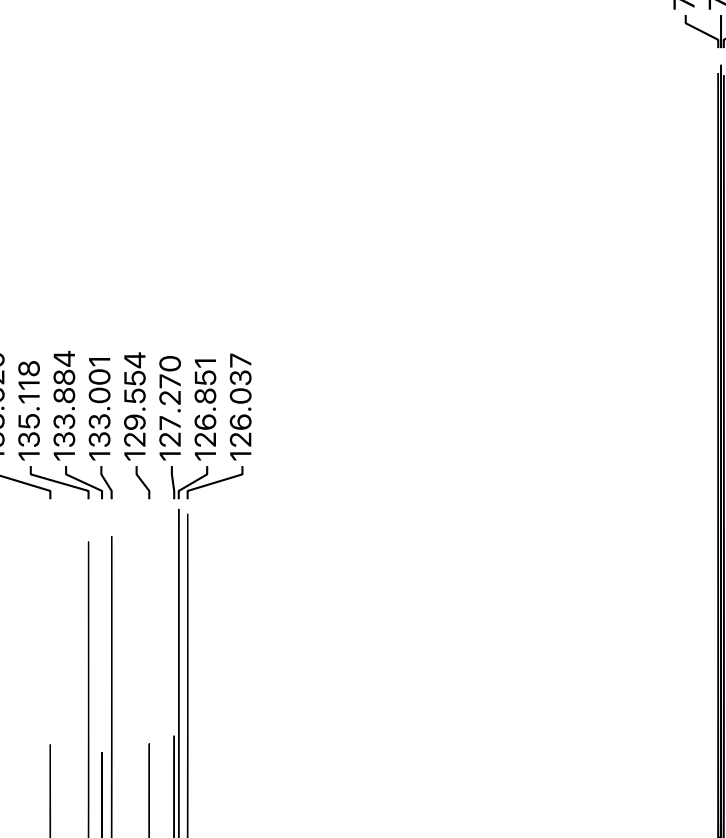

\begin{tabular}{|c|c|c|c|c|c|c|c|c|c|c|c|}
\hline 220 & 200 & 180 & 160 & 140 & 120 & 100 & 80 & 60 & 40 & 20 & $\begin{array}{c}0 \\
\text { Page } \$ 66\end{array}$ \\
\hline
\end{tabular}


는

$\infty \infty \infty$

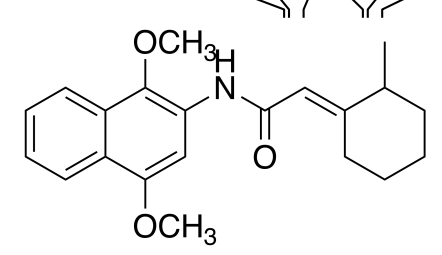

S10, ${ }^{1} \mathrm{H}$ NMR $\left(500 \mathrm{MHz}, \mathrm{CDCl}_{3}\right)$

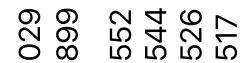

(1)

li njm

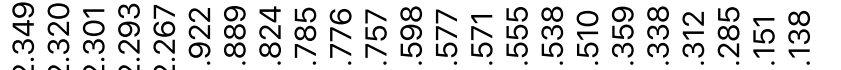

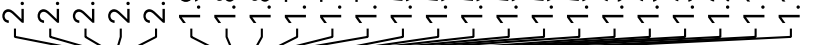

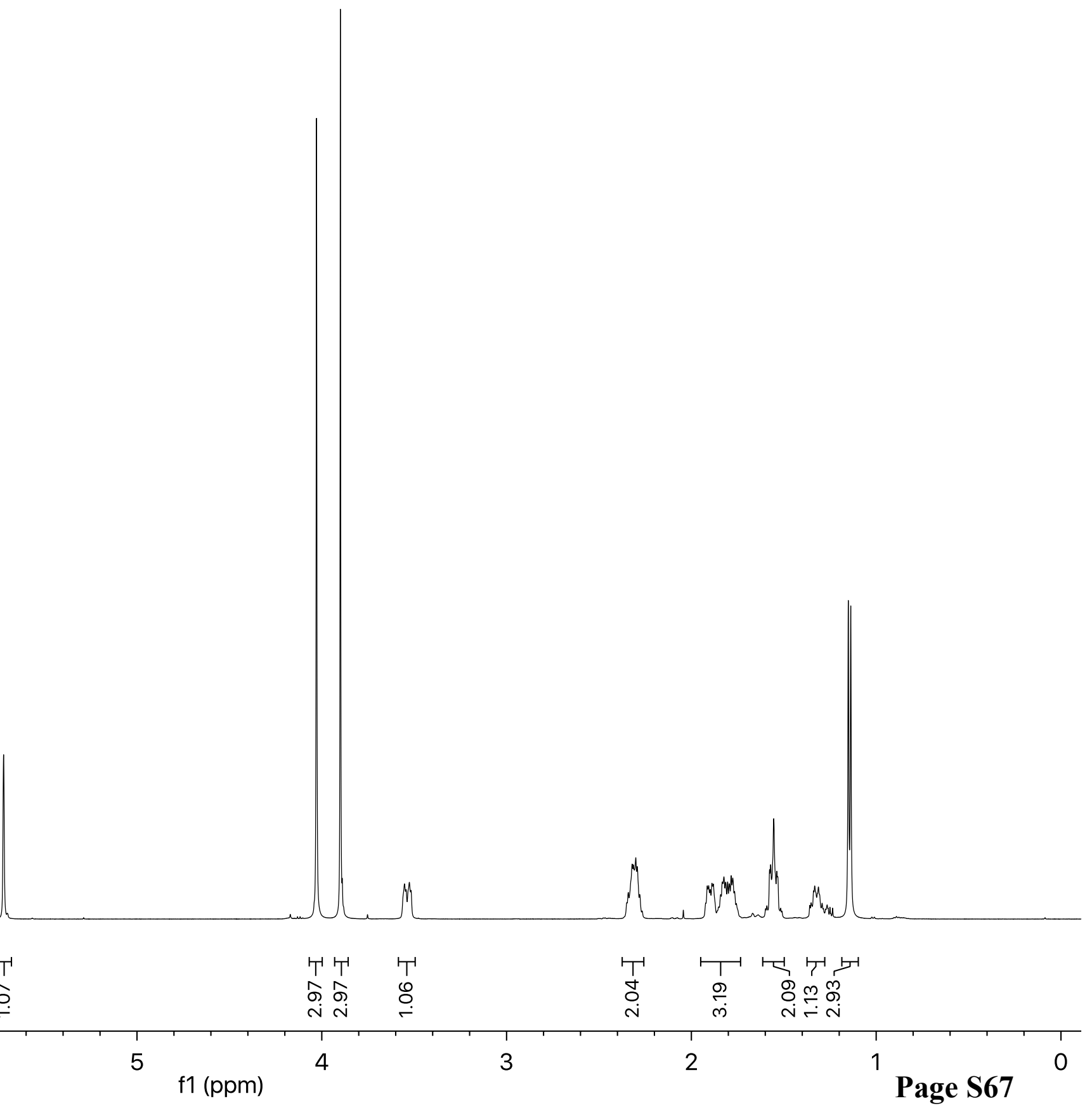




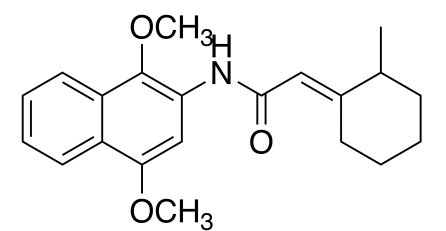

S10, ${ }^{13} \mathrm{C} \mathrm{NMR}\left(126 \mathrm{MHz}, \mathrm{CDCl}_{3}\right)$

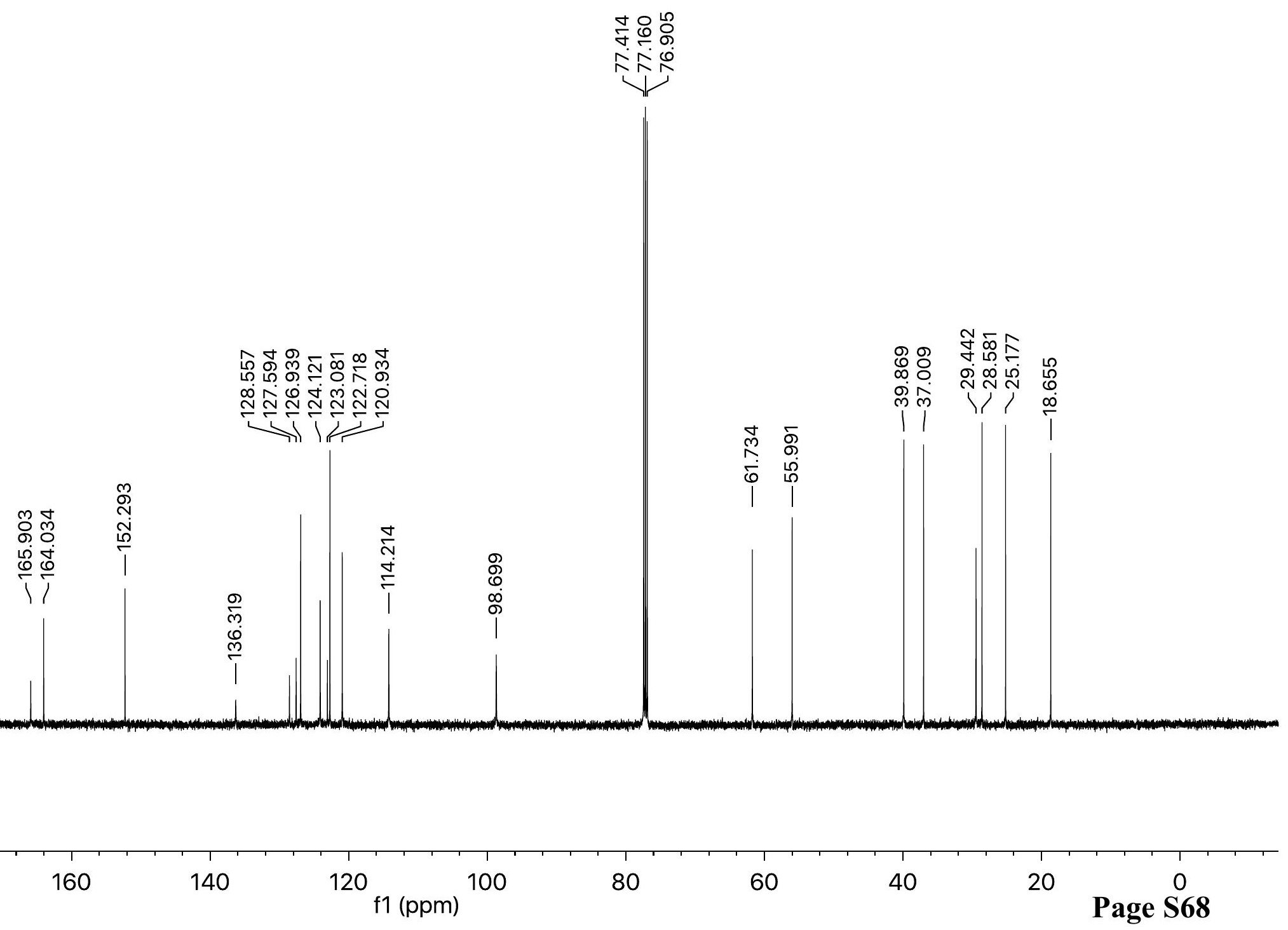




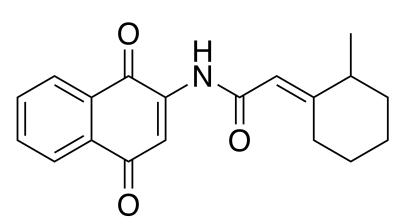

37, ${ }^{1} \mathrm{H}$ NMR (500 MHz, $\mathrm{CDCl}_{3}$ )

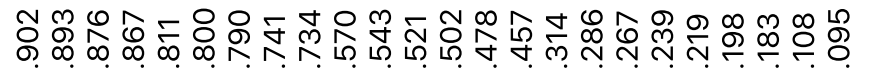 \\ 亡}

iाiाn

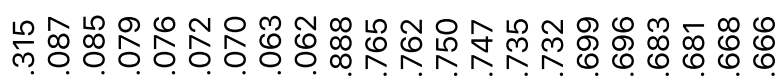

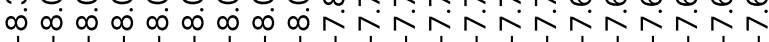

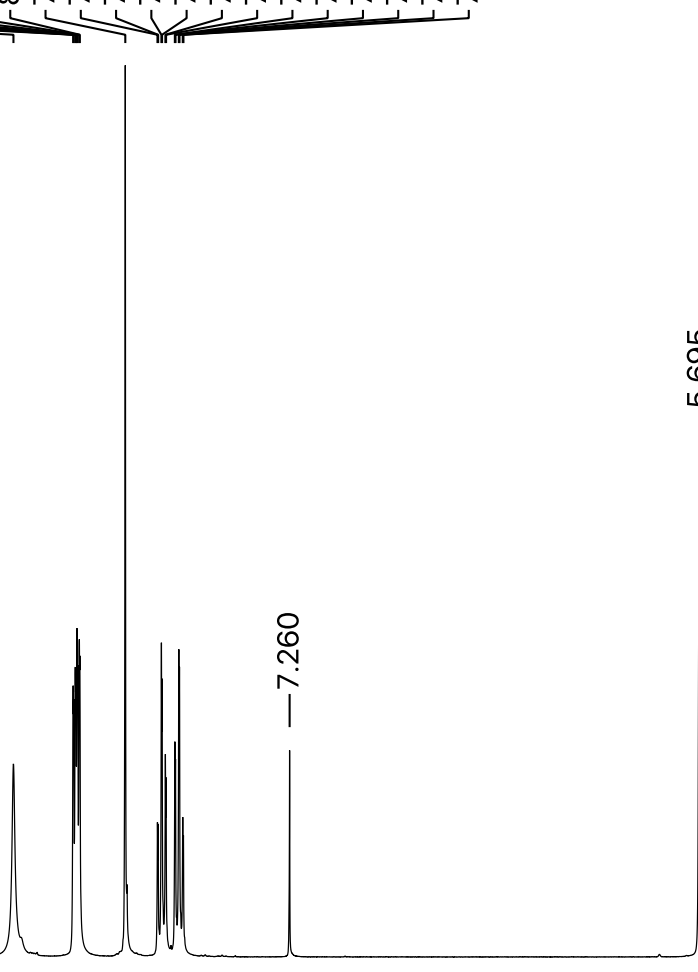

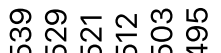

ஸे लं

m m

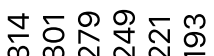

ง ำ ํำ

Niven

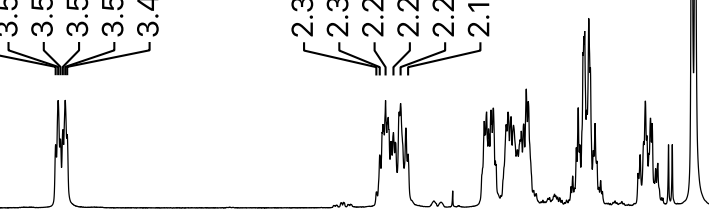

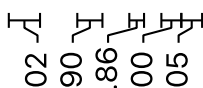

T

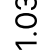

T

एएँ

o

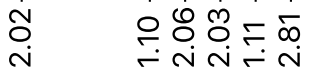




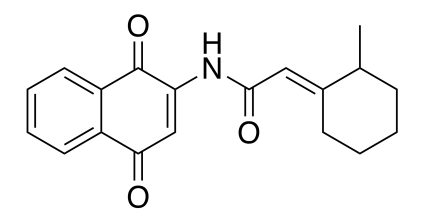

37, ${ }^{13} \mathrm{C} \mathrm{NMR}\left(126 \mathrm{MHz}, \mathrm{CDCl}_{3}\right)$

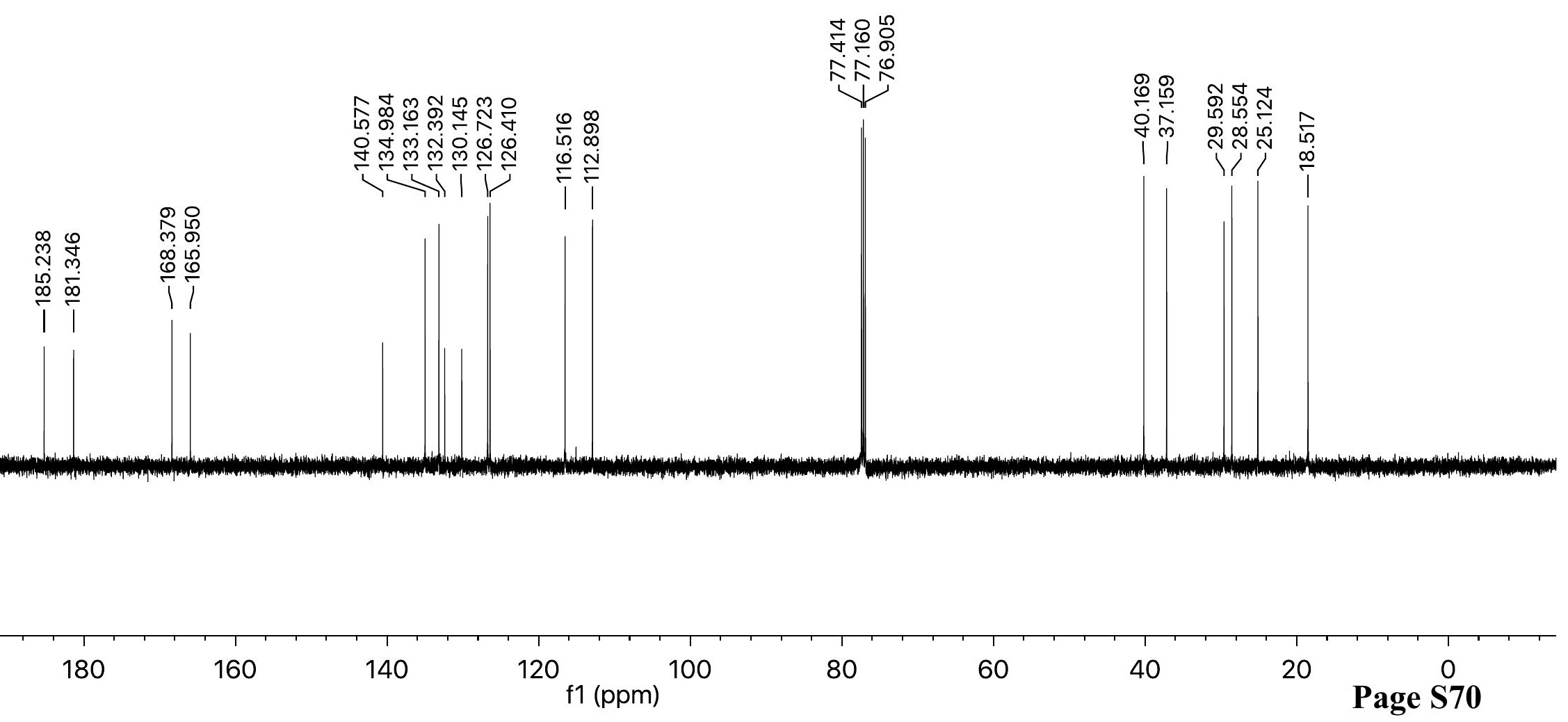




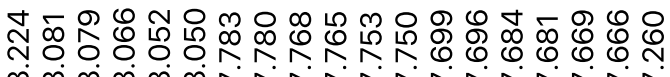

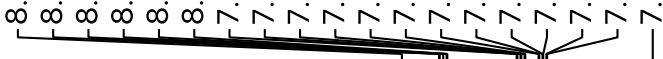

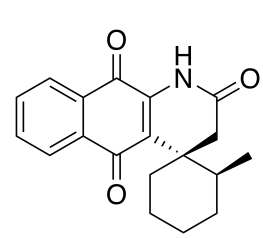

38, ${ }^{1} \mathrm{H}$ NMR $\left(500 \mathrm{MHz}, \mathrm{CDCl}_{3}\right)$

\section{8,}

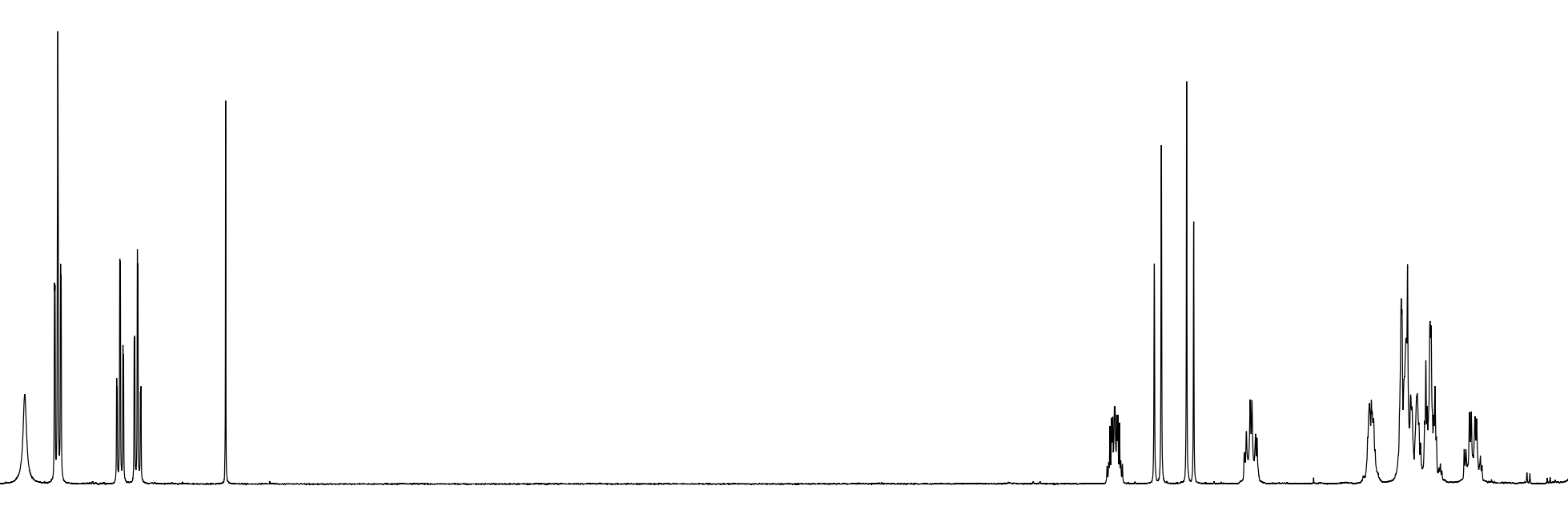

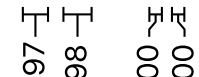

임
\end{abstract}

8
TH 읃윰욛

3
머ㄴㅏㅏ ㄷำ ำ $r$
T
$\stackrel{+}{\mathrm{N}}$ 


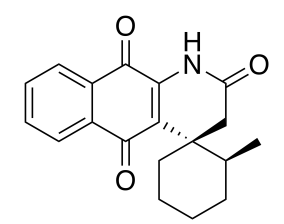

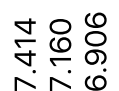

숭

38, ${ }^{13} \mathrm{C} \mathrm{NMR}\left(126 \mathrm{MHz}, \mathrm{CDCl}_{3}\right)$

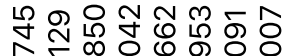

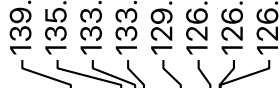

|

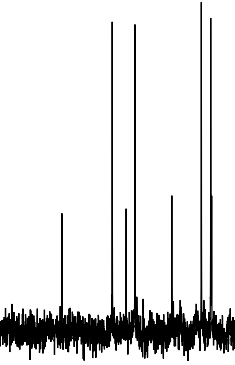

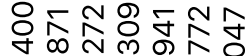

ம் में

ifiti

I্ণ

\begin{tabular}{llllllllll}
\hline 220 & 200 & 180 & 160 & 140 & 120 & 100 & 80 & 60 & 40
\end{tabular}




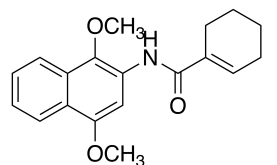

S11, ${ }^{1} \mathrm{H}$ NMR $\left(500 \mathrm{MHz}, \mathrm{CDCl}_{3}\right)$
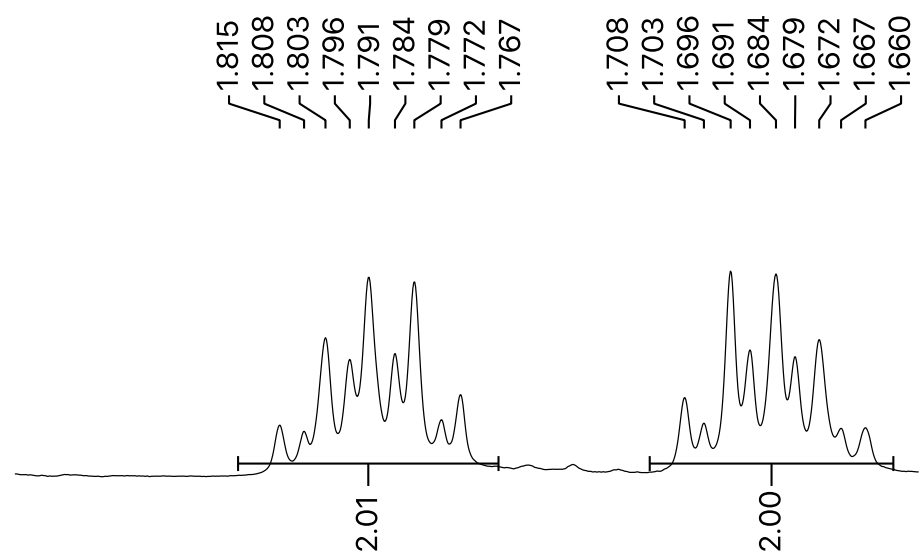

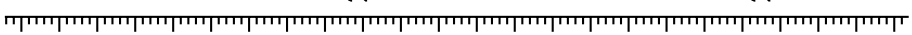
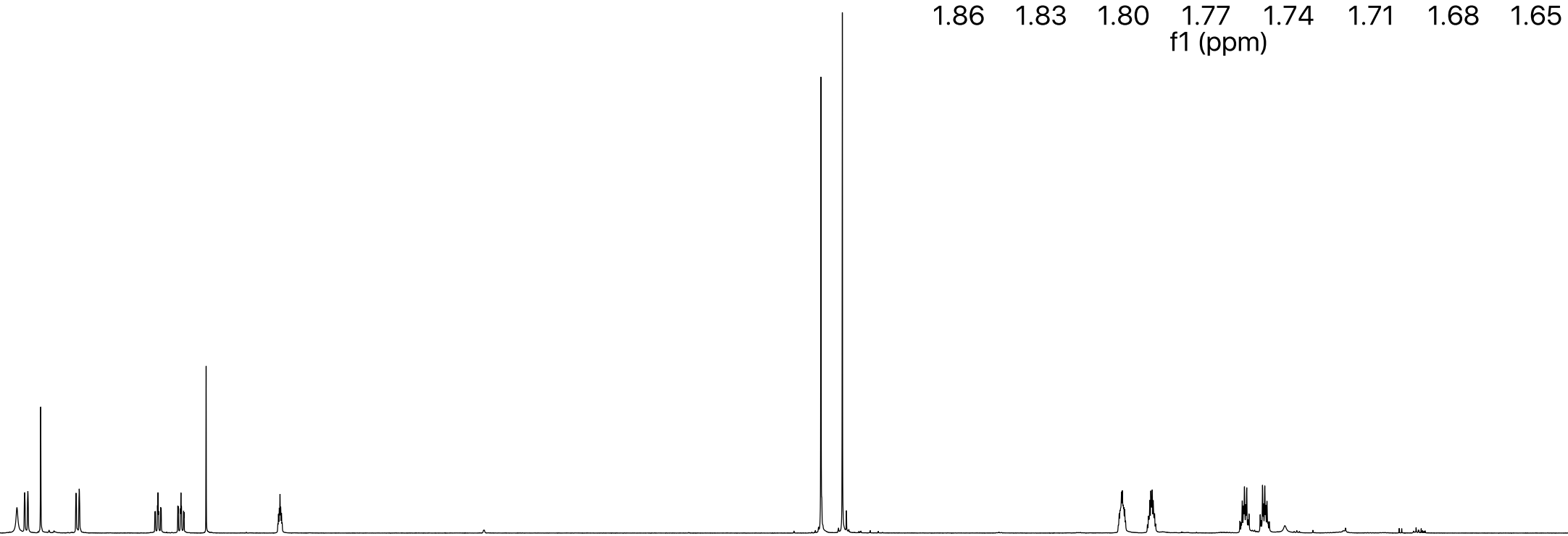

\begin{tabular}{|c|c|c|c|c|c|c|}
\hline & & 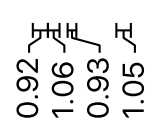 & 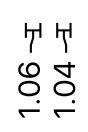 & $\begin{array}{l}\text { T' } \\
\text { ô } \\
0\end{array}$ & & \\
\hline 10 & 9 & 8 & & 7 & 6 & $\begin{array}{c}5 \\
\text { f1 (ppm) }\end{array}$ \\
\hline
\end{tabular}

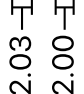

TT'T

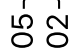

in

4

3

2

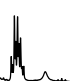

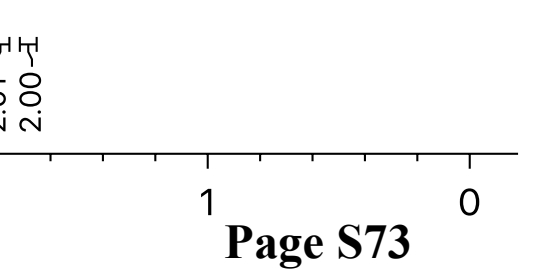




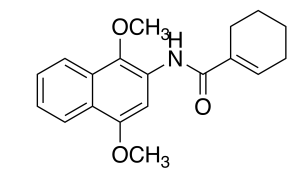

S11, ${ }^{13} \mathrm{C}$ NMR $\left(126 \mathrm{MHz}, \mathrm{CDCl}_{3}\right)$

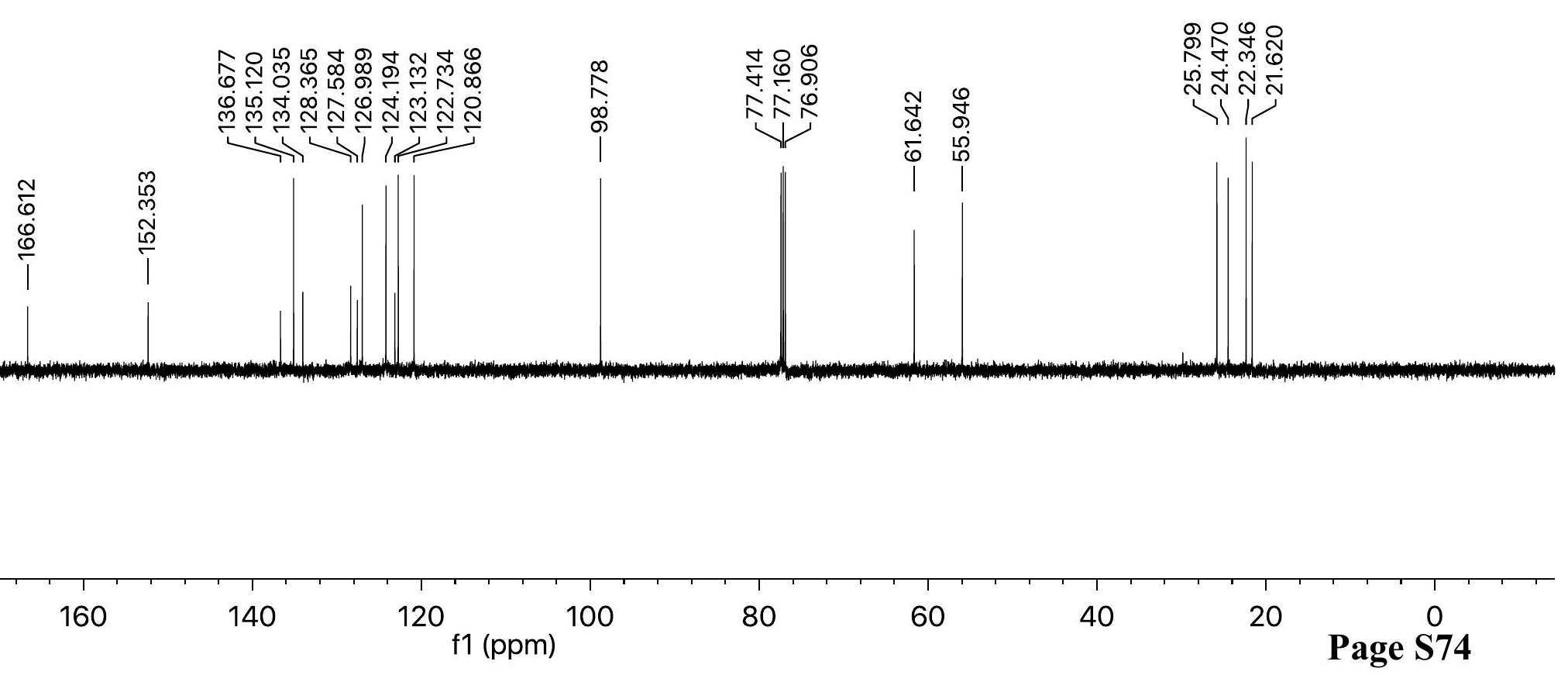




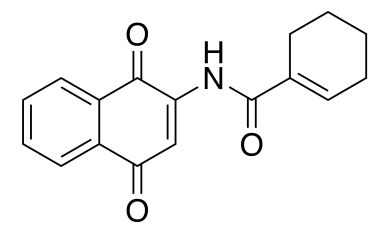

39, ${ }^{13} \mathrm{C} \mathrm{NMR}\left(126 \mathrm{MHz}, \mathrm{CDCl}_{3}\right)$

号용

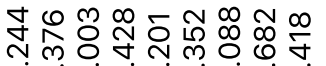

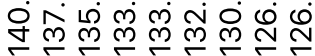

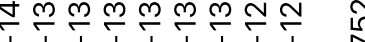

$\stackrel{\infty}{\sim} \stackrel{0}{0}$

ஸें

$\infty$

i

$\infty$

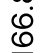

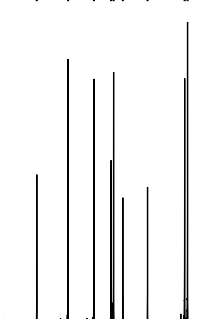

송

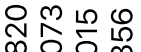

भi.

กู่

\section{$\frac{1}{2}$}

i
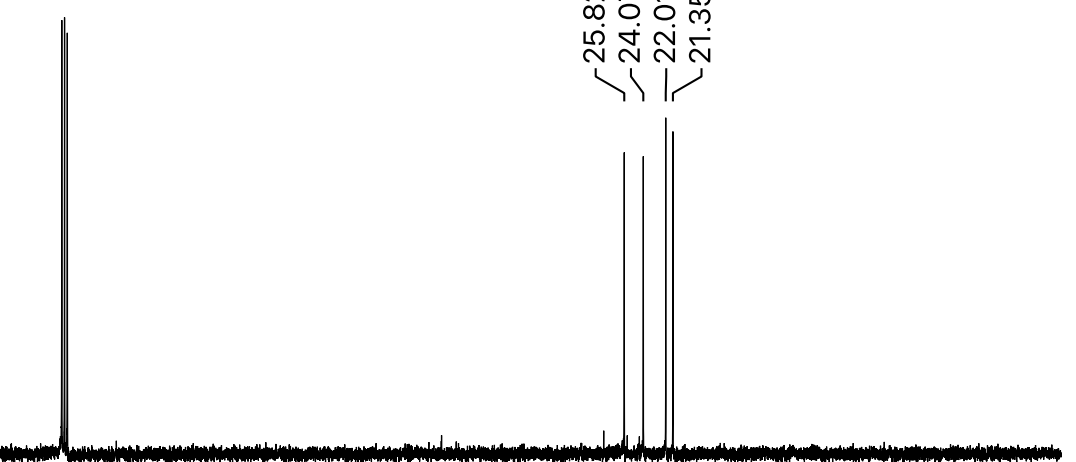

$\begin{array}{llllllllll} & 200 & 180 & 160 & 140 & 120 & 100 & 80 & 60 & 40\end{array}$




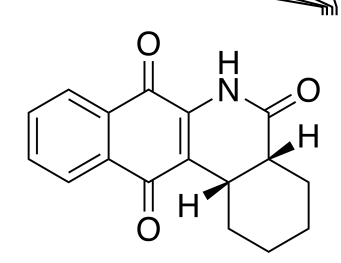

40, ${ }^{1} \mathrm{H}$ NMR $\left(500 \mathrm{MHz}, \mathrm{CDCl}_{3}\right)$

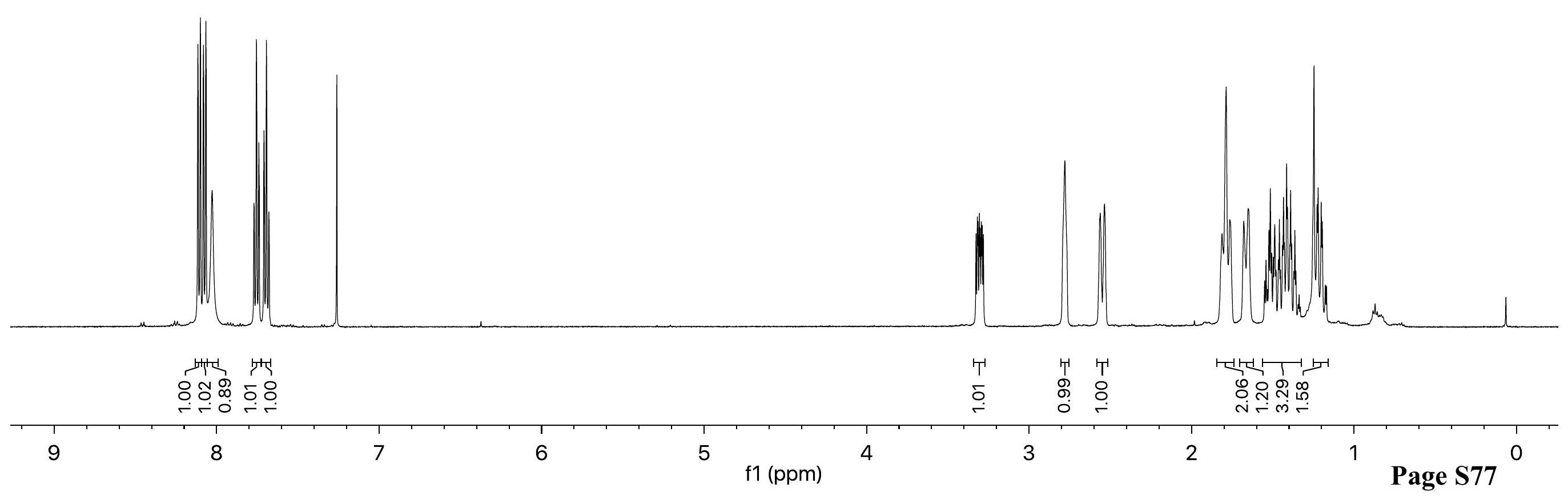




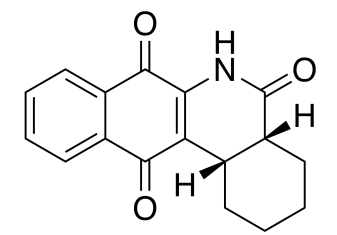

40, ${ }^{13} \mathrm{C} \mathrm{NMR}\left(126 \mathrm{MHz}, \mathrm{CDCl}_{3}\right)$

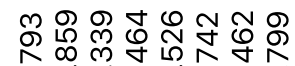

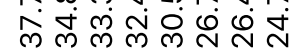

迕行

$\stackrel{\circ}{\stackrel{\circ}{\circ}} \stackrel{8}{\circ}$

추용

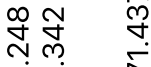

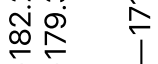

I 1

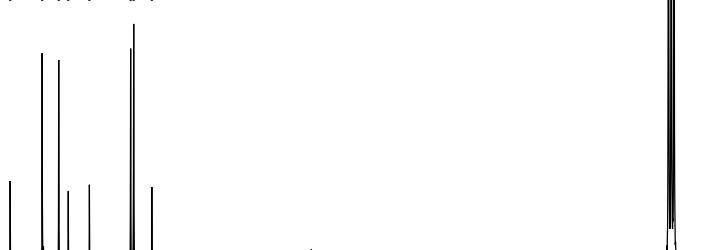

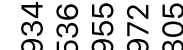

๘ लंล่

०)

|

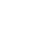

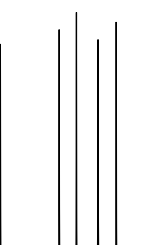

W.

\begin{tabular}{lllllllllll}
\hline 20 & 200 & 180 & 160 & 140 & 120 & 100 & 80 & 60 & 40 & \\
f1 (ppm) & & & &
\end{tabular}




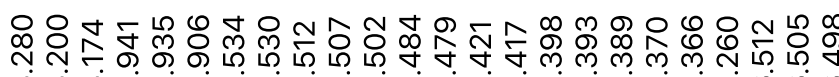

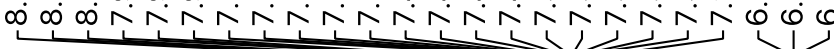

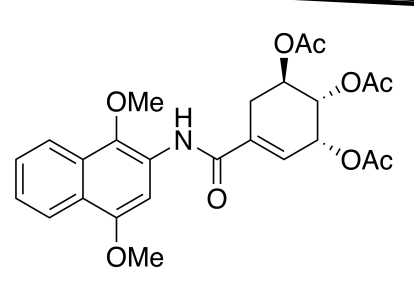

S12, ${ }^{1} \mathrm{H}$ NMR $\left(500 \mathrm{MHz}, \mathrm{CDCl}_{3}\right)$

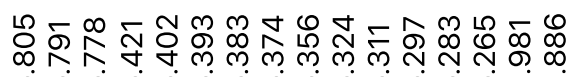

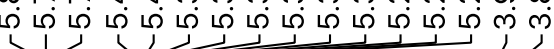

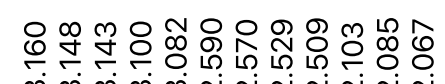

ming miñ

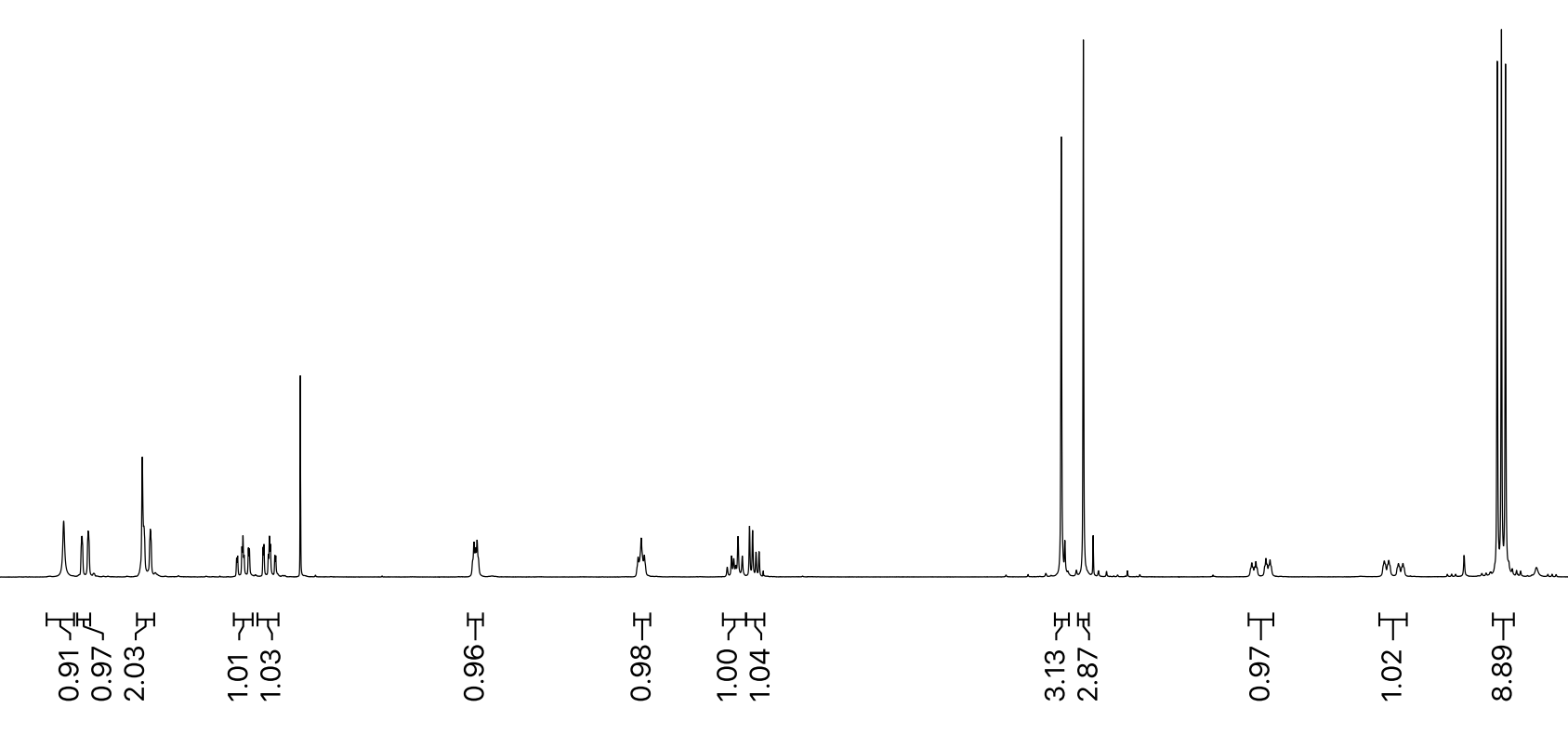

10

9

8

7

6

5

f1 (ppm)

4

3

2

1

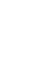

0

Page S79 


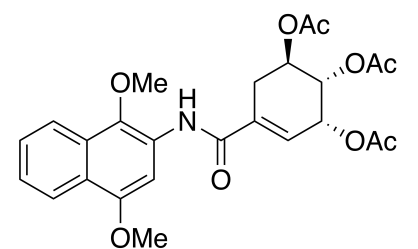

S12, ${ }^{13} \mathrm{C}$ NMR (126 MHz, $\mathrm{CDCl}_{3}$ )

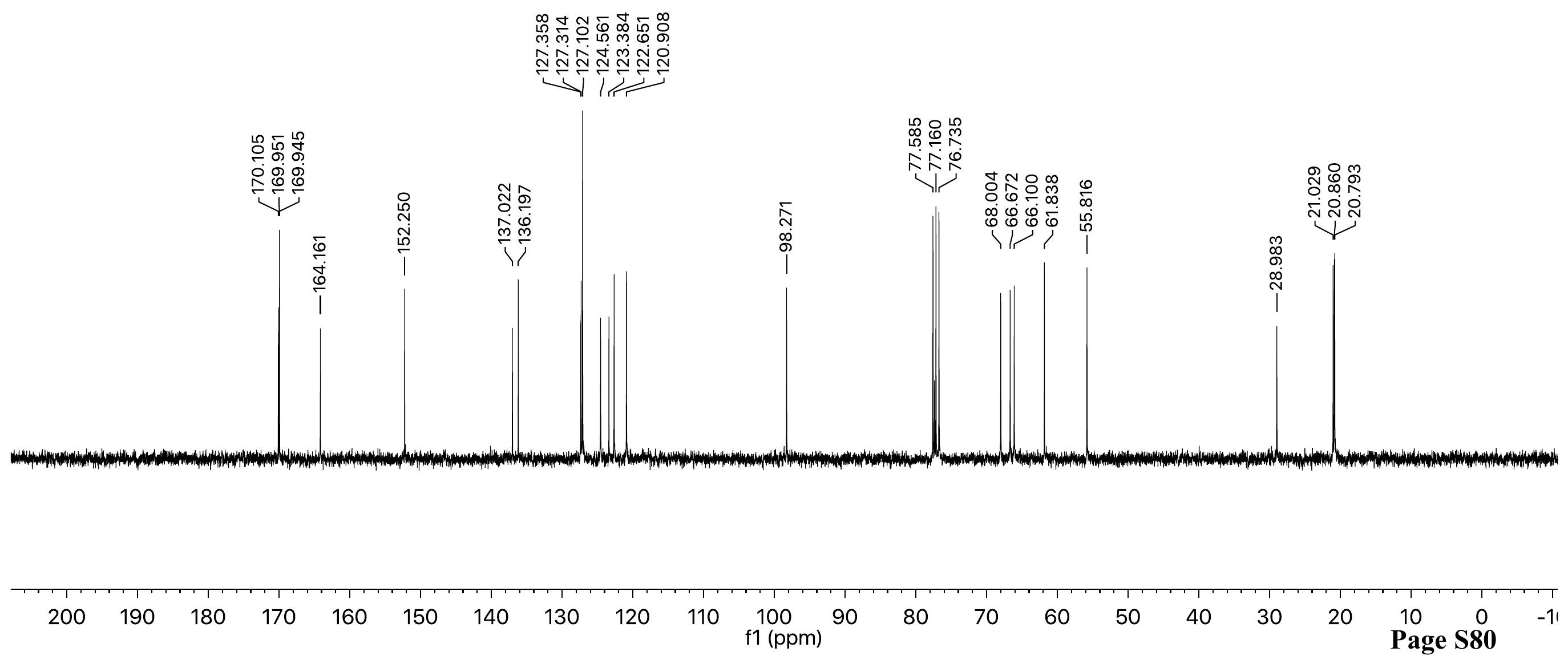




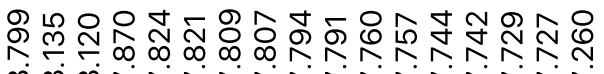

ㅈํㅇ온 운

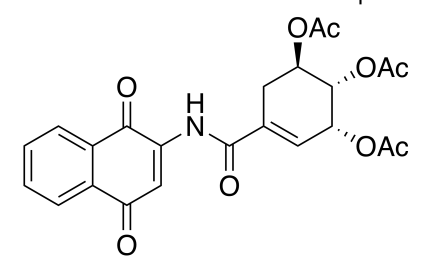

41, ${ }^{1} \mathrm{H}$ NMR $\left(500 \mathrm{MHz}, \mathrm{CDCl}_{3}\right)$

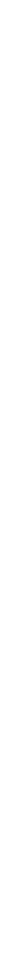




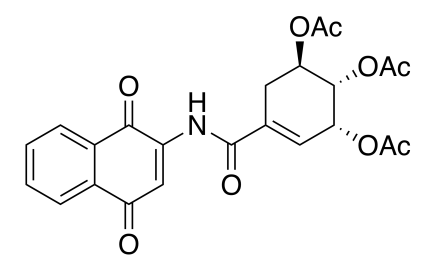

41, ${ }^{13} \mathrm{C}$ NMR $\left(126 \mathrm{MHz}, \mathrm{CDCl}_{3}\right)$

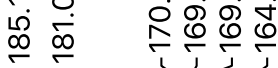

$$
-
$$




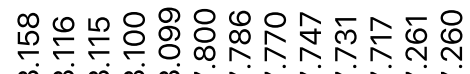

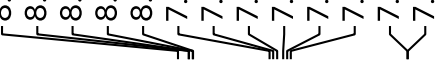

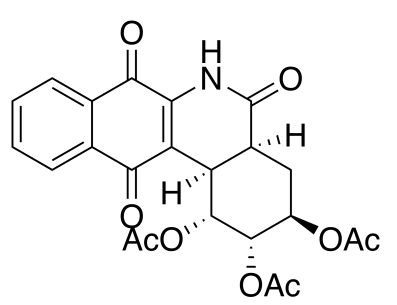

42, ${ }^{1} \mathrm{H}$ NMR (500 MHz, $\left.\mathrm{CDCl}_{3}\right)$

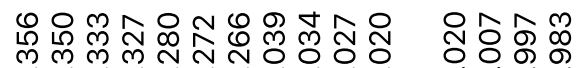

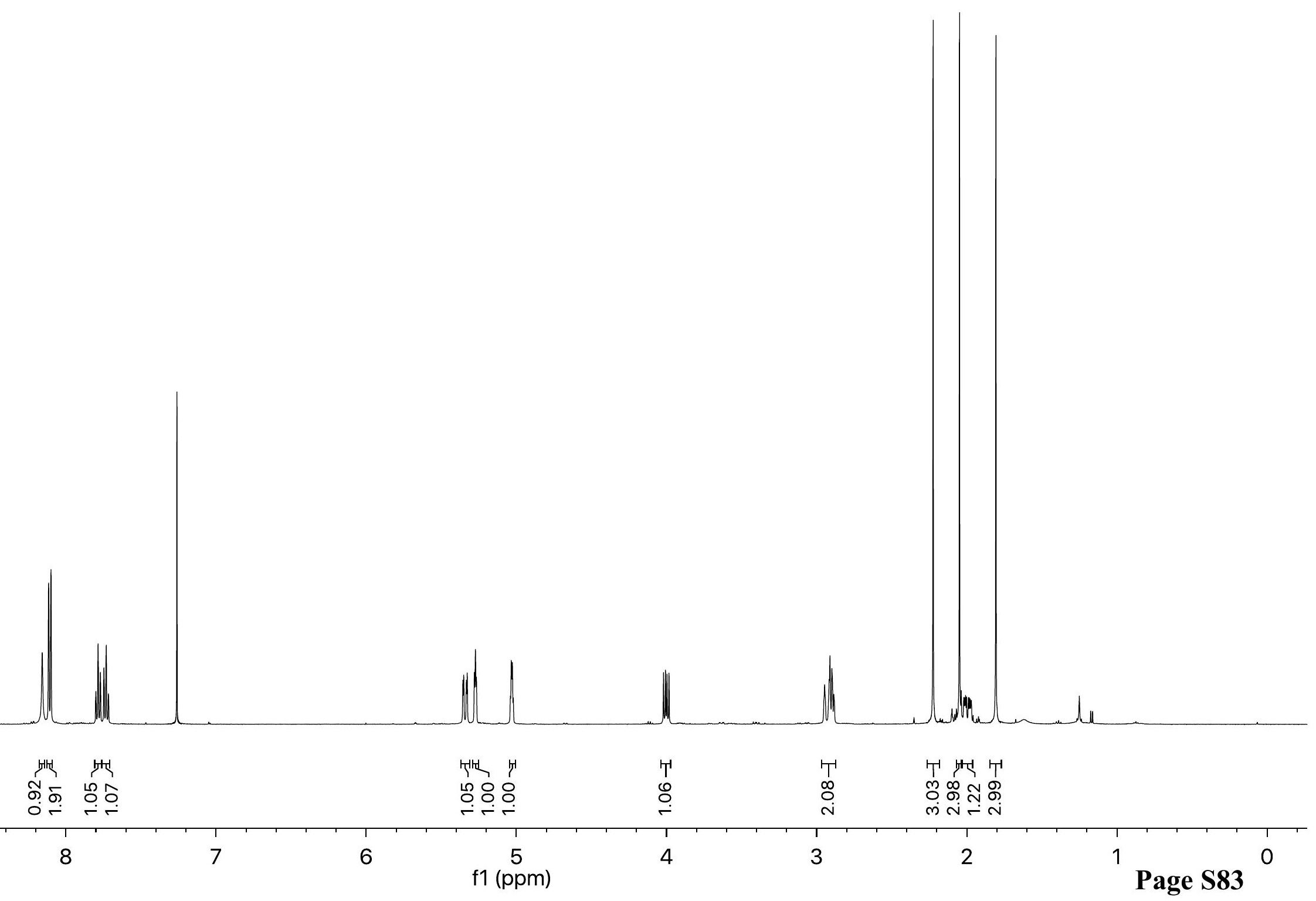




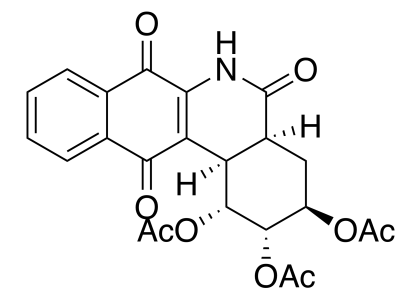

42, ${ }^{13} \mathrm{C}$ NMR $\left(126 \mathrm{MHz}, \mathrm{CDCl}_{3}\right)$

\begin{tabular}{lllllllllll}
\hline 20 & 200 & 180 & 160 & 140 & 120 & 100 & 80 & 60 & 40 & \\
f1 (ppm) & & & &
\end{tabular}




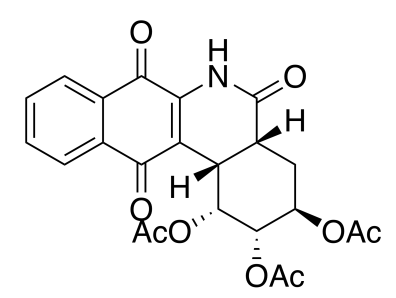

43, ${ }^{13} \mathrm{C}$ NMR (126 MHz, $\mathrm{CDCl}_{3}$ )

オ

Nं

皮

品 


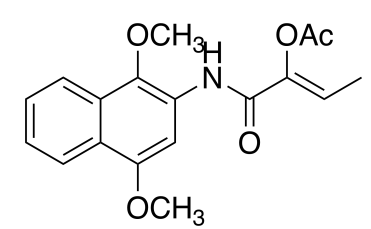

S13, ${ }^{1} \mathrm{H}$ NMR $\left(500 \mathrm{MHz}, \mathrm{CDCl}_{3}\right)$

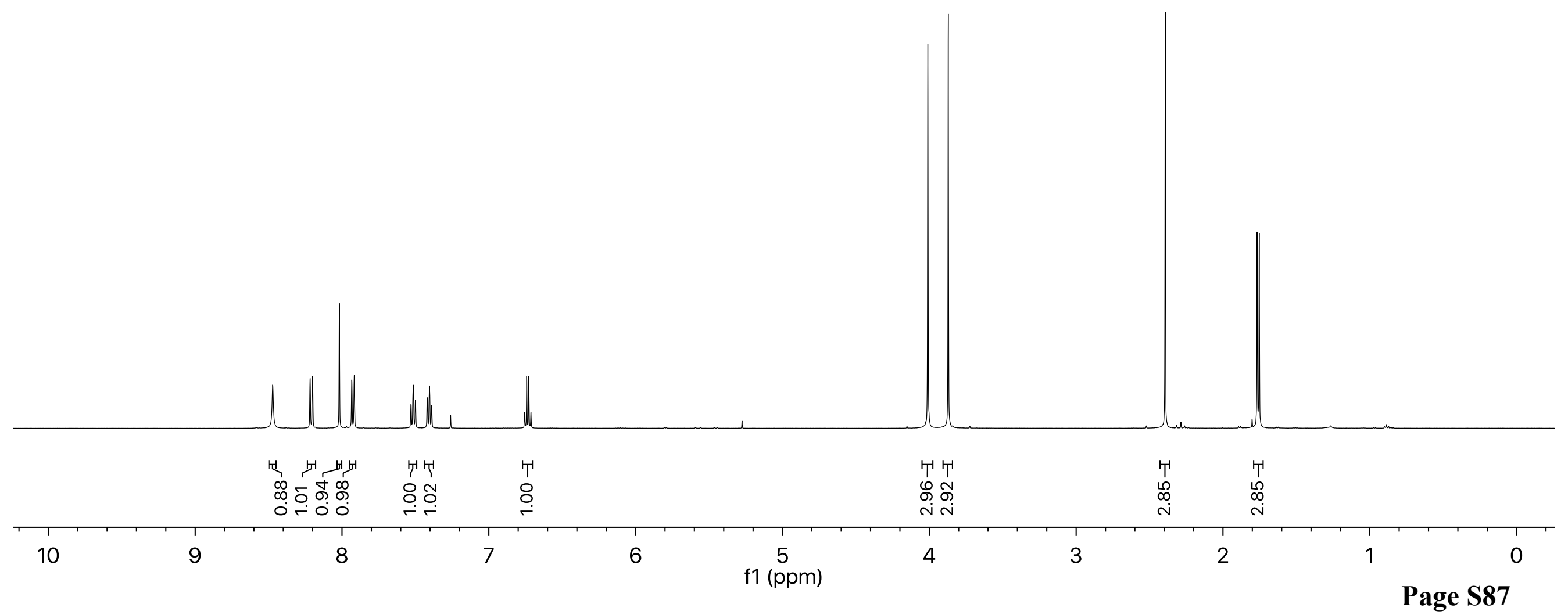




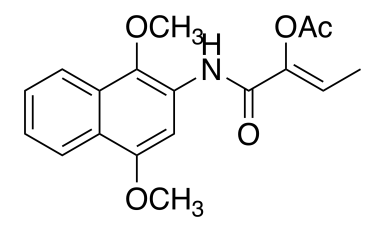

S13, ${ }^{13} \mathrm{C} \mathrm{NMR}\left(126 \mathrm{MHz}, \mathrm{CDCl}_{3}\right)$
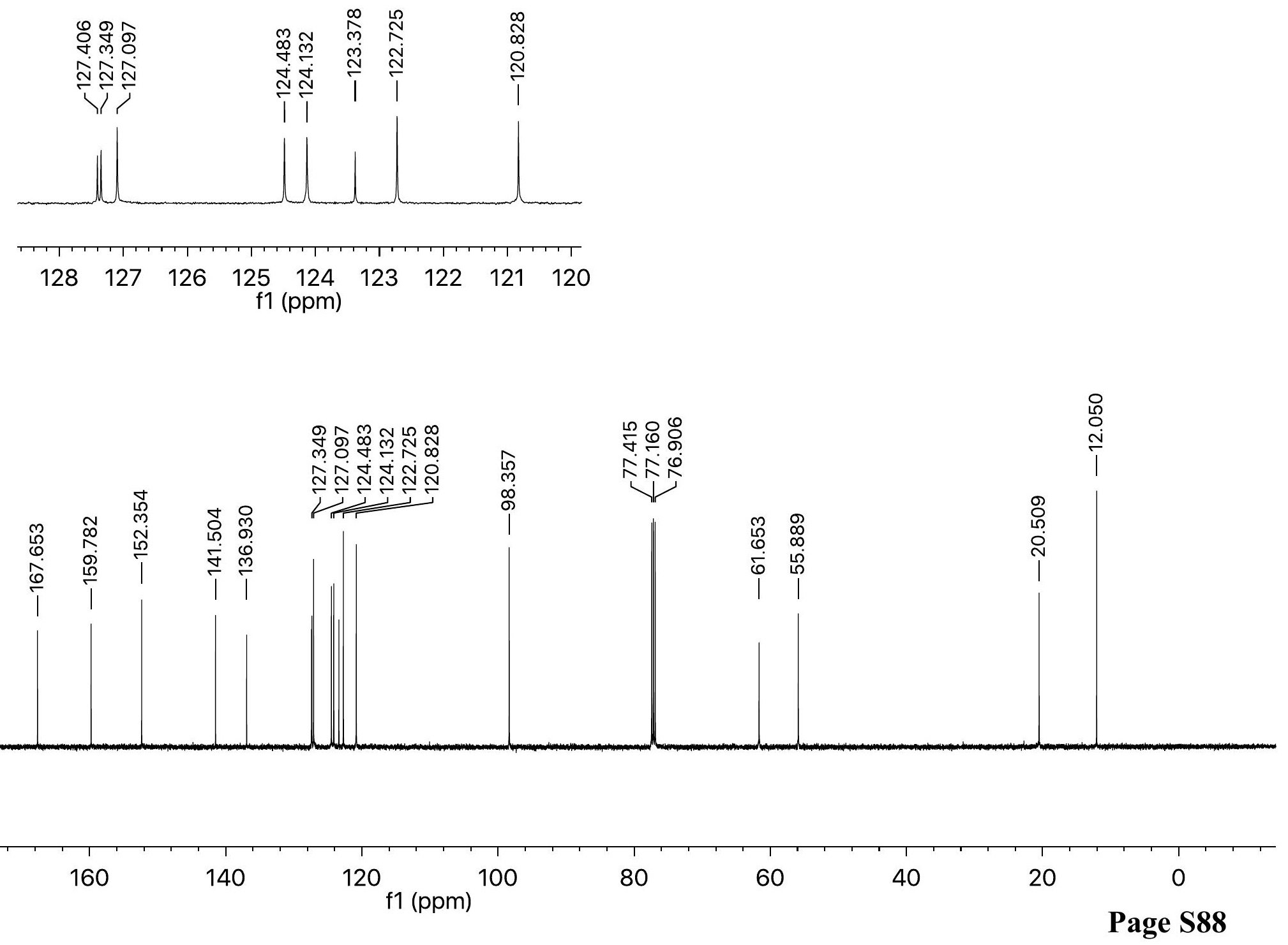


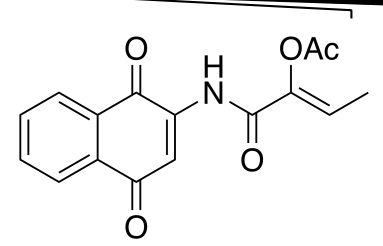

45, ${ }^{1} \mathrm{H}$ NMR $\left(500 \mathrm{MHz}, \mathrm{CDCl}_{3}\right)$

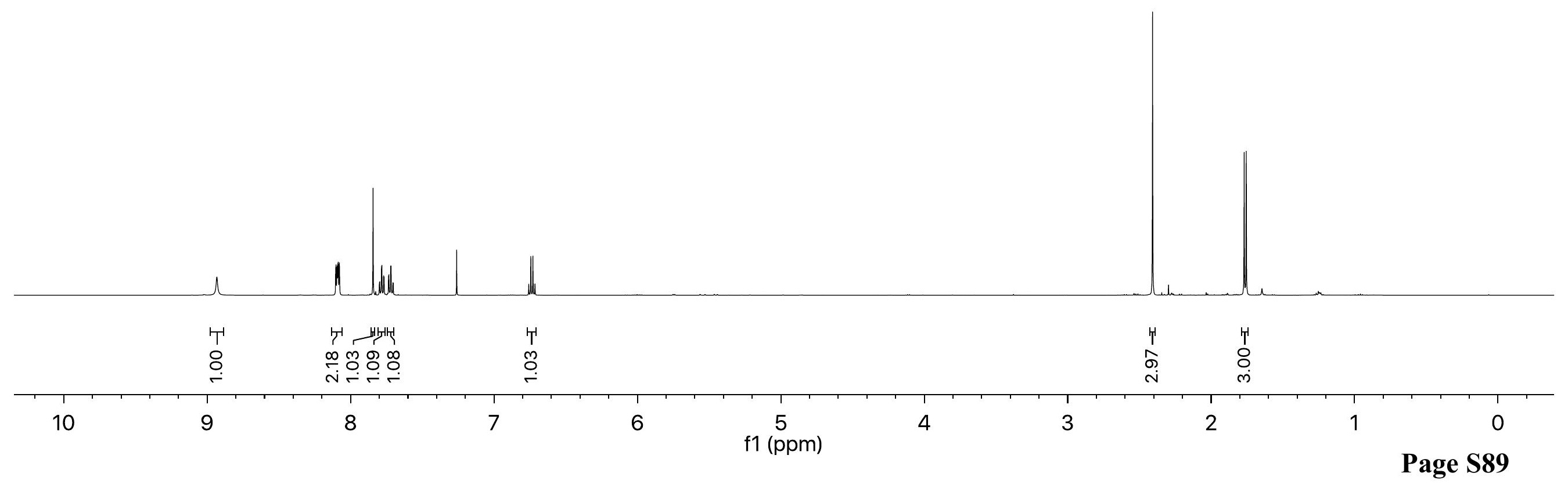




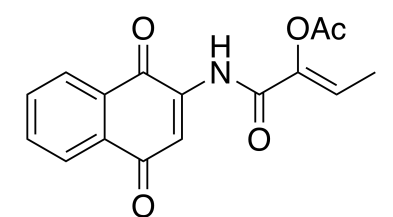

45, ${ }^{13} \mathrm{C}$ NMR $\left(126 \mathrm{MHz}, \mathrm{CDCl}_{3}\right)$

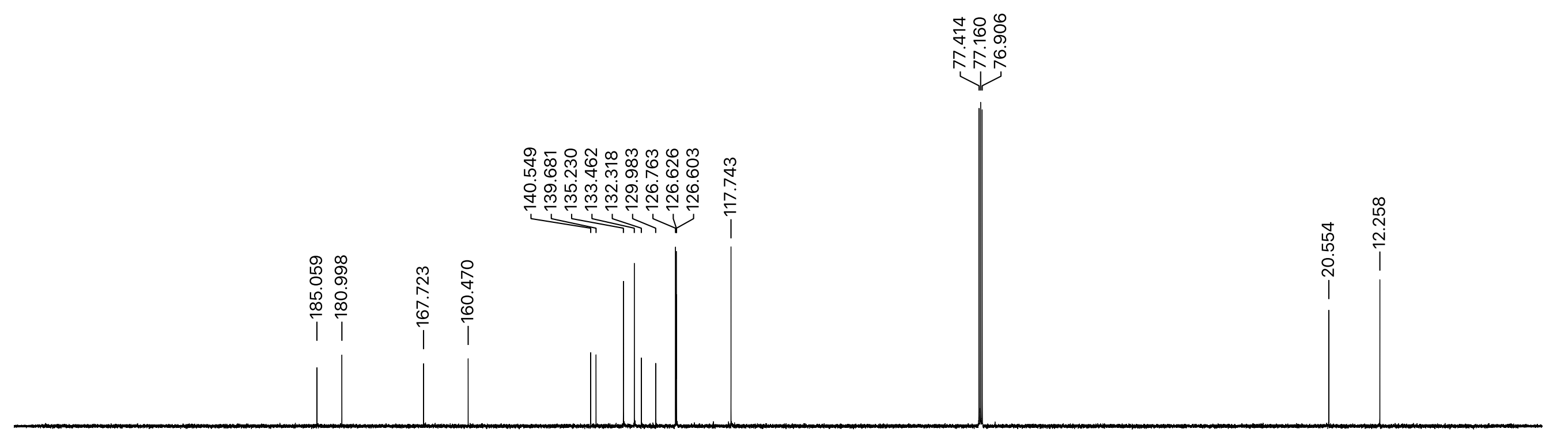




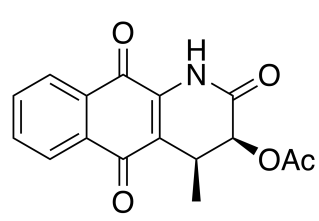

47, ${ }^{1} \mathrm{H}$ NMR (500 MHz, $\mathrm{CDCl}_{3}$ )

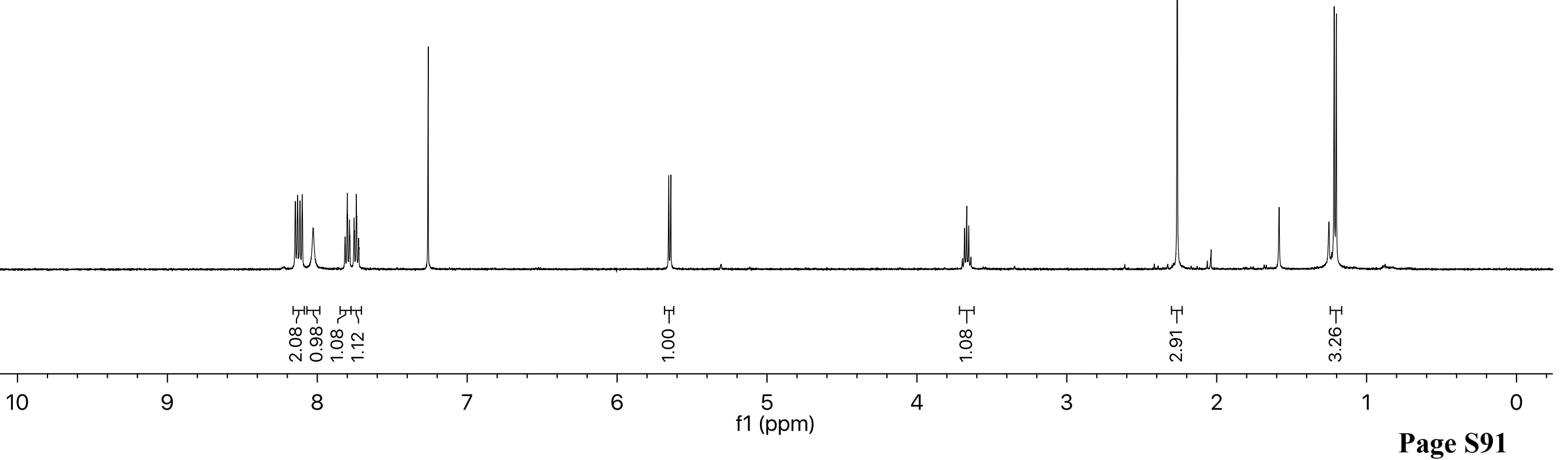




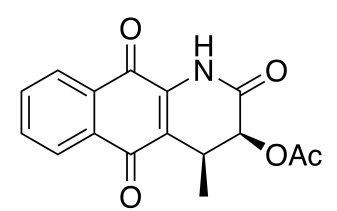

47, ${ }^{13} \mathrm{C}$ NMR $\left(126 \mathrm{MHz}, \mathrm{CDCl}_{3}\right)$

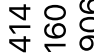

동

证

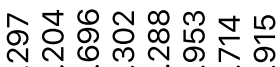

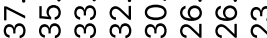

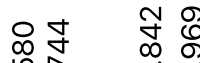

$\stackrel{\infty}{\infty} \stackrel{\circ}{\circ}$

I

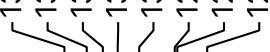

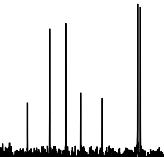

180

160

140

120

f1 (ppm) 


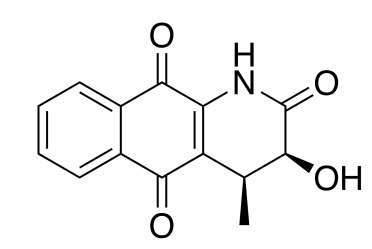

( \pm Griffithazanone A, ${ }^{1} \mathrm{H}$ NMR (500 MHz, $\left.\mathrm{CDCl}_{3}\right)$

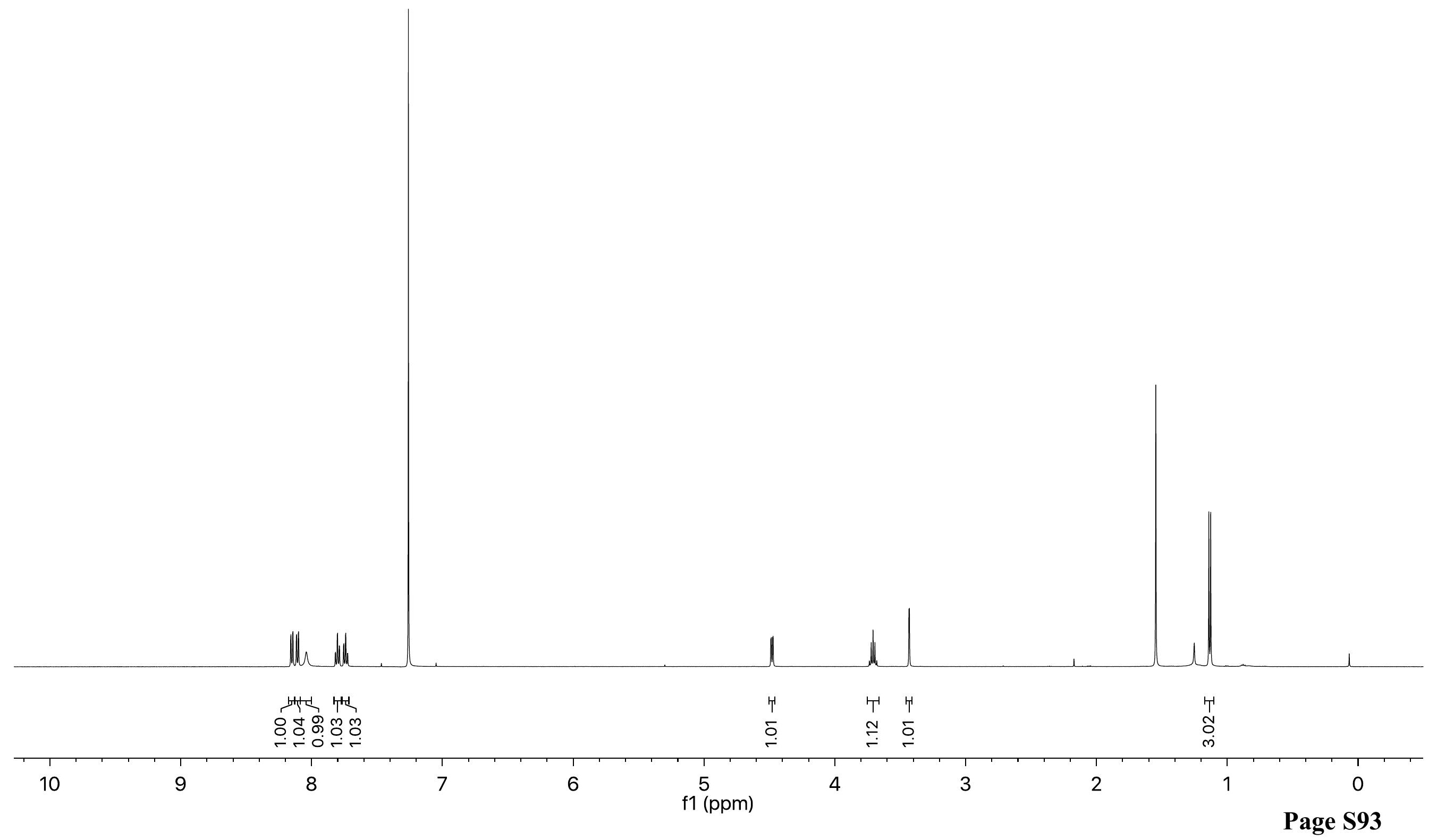




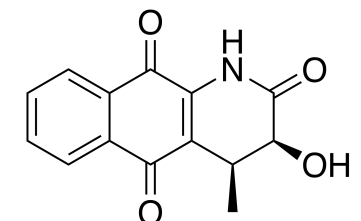

( \pm Griffithazanone $\mathbf{A},{ }^{13} \mathrm{C} \mathrm{NMR}\left(126 \mathrm{MHz}, \mathrm{CDCl}_{3}\right)$

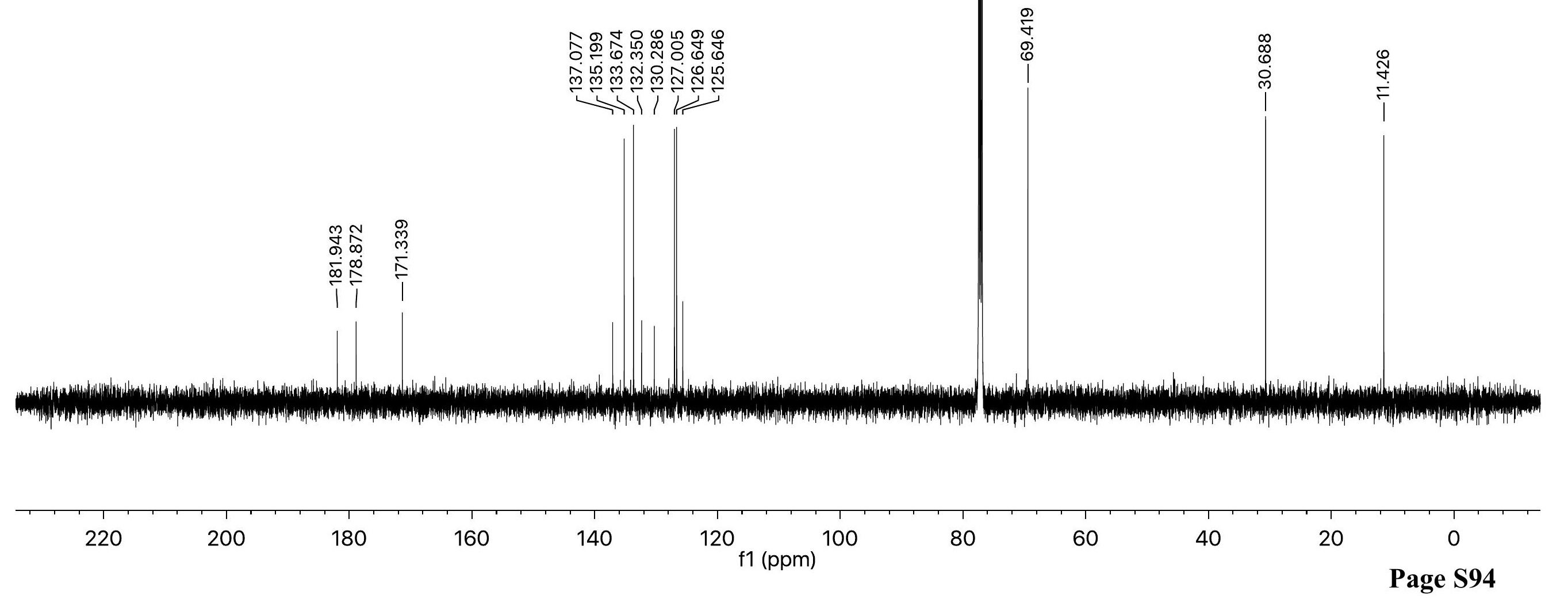




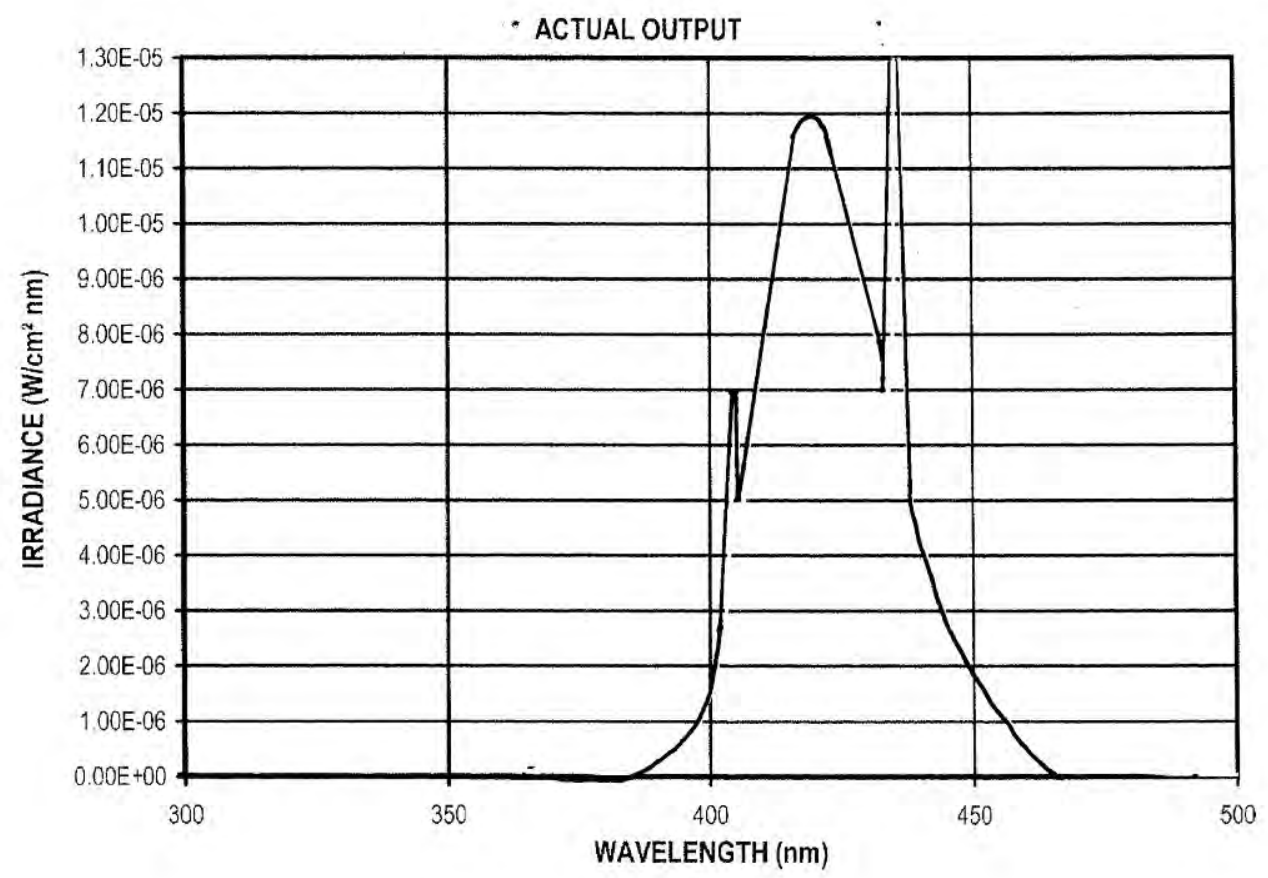

Fig S1: spectral distribution and intensity of the light source RPR-4190A. 


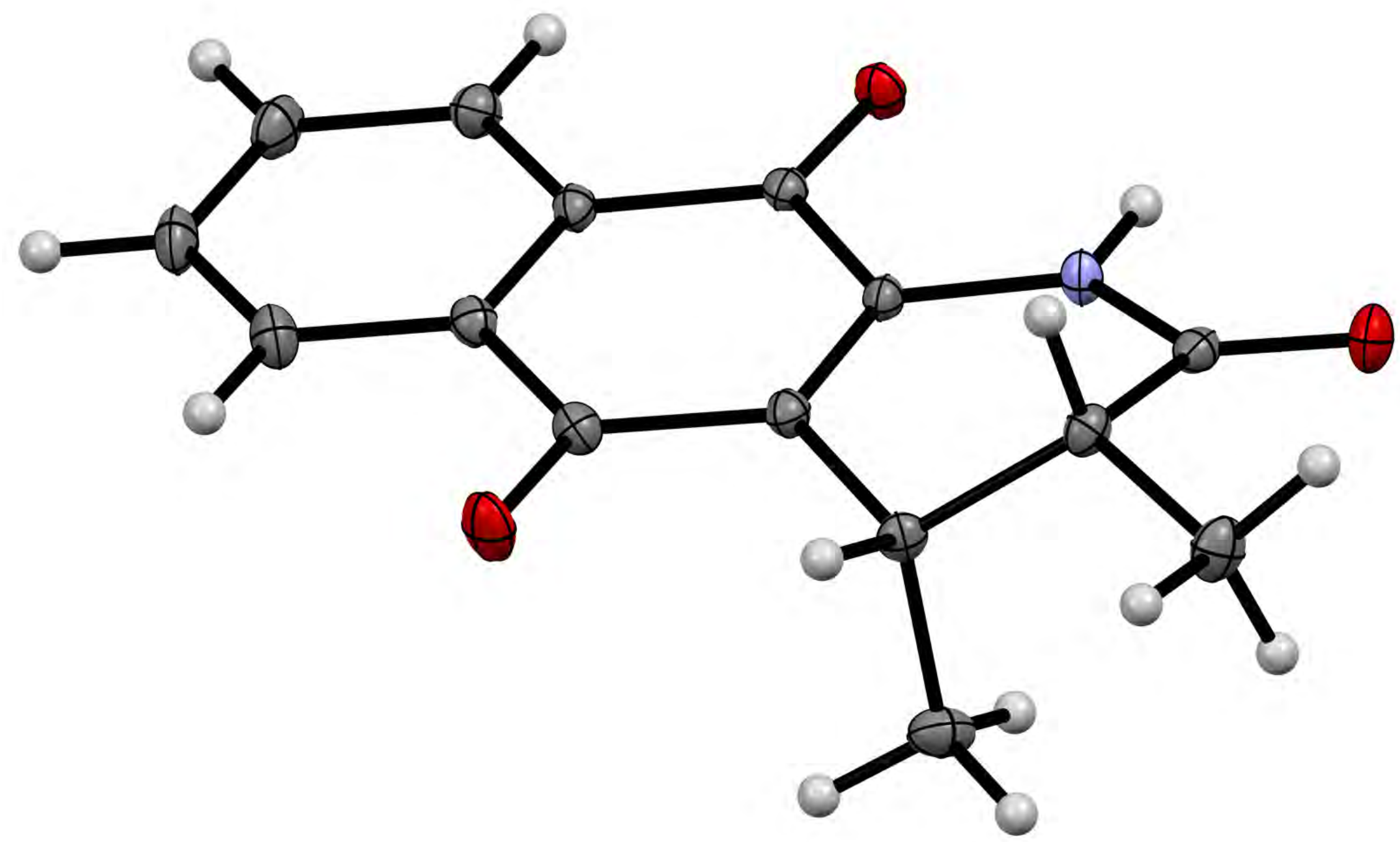

Figure S2: Crystal structure of 14. The ellipsoid contour percent probability of $50 \%$ was used for the caption of this image. 


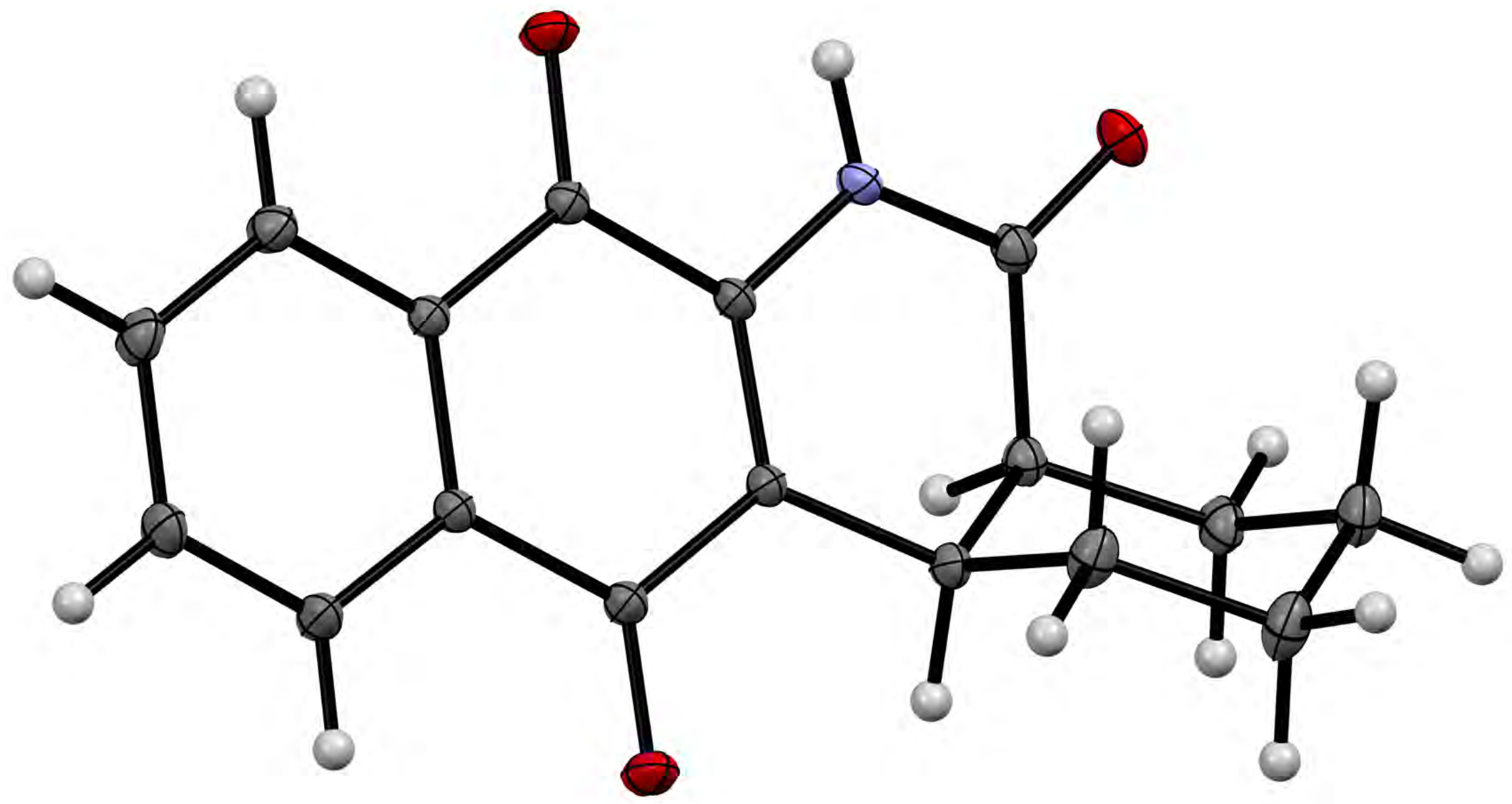

Figure S3: Crystal structure of 40. The ellipsoid contour percent probability of 50\% was used for the caption of this image. 
XRD sample preparation of compound 14. Compound $14(5 \mathrm{mg})$ was dissolved in $\mathrm{MeOH}(2 \mathrm{~mL})$ in a $5 \mathrm{~mL}$ scintillation vial. On the top of the resulting solution was carefully added hexanes $(2 \mathrm{~mL})$ so that there were 2 layers. The uncapped scintillation vial was placed in the center of a larger chamber filled with hexanes (about $10 \mathrm{~mL}$ ). The outer chamber was capped. 6 months later all of the solvents had escaped and the resulting crystal was subjected to X-ray diffraction.

XRD sample preparation of compound 40. Compound $40(5 \mathrm{mg})$ was dissolved in $\mathrm{CHCl}_{3}(\sim 0.2 \mathrm{~mL})$ in a $5 \mathrm{~mL}$ scintillation vial. On the top of the resulting solution was carefully added hexanes $(2 \mathrm{~mL})$ so that there were 2 layers. The uncapped scintillation vial was placed in the center of a larger chamber filled with hexanes (about $10 \mathrm{~mL}$ ). The outer chamber was capped. 6 months later all of the solvents had escaped and the resulting crystal was subjected to X-ray diffraction.

Crystal Structure Determination. Single-crystal X-ray diffraction data was collected on a Nonius Kappa diffractometer equipped with Mo K $\alpha$ radiation $(\lambda=0.71073 \AA)$ and a BRUKER APEXII CCD. The APEX31 software suite was used to manage data collection, reduction (SAINT V8.38A ${ }^{1}$ ), absorption correction by the Multi-scan method (SADABS ${ }^{2}$ ), structure determination via direct methods $\left(\mathrm{SHLEXT}^{3}\right)$ and model refinement $\left(\mathrm{SHELXL}^{4}\right)$. All data were collected at 103(2) K. All non-hydrogen atoms were refined anisotropically. All hydrogen atoms, with the exception of those bound to nitrogen, were refined with their positions ideally constrained to their carriers. Hydrogen bonded with nitrogen were refined isotropically with restrained bond lengths. The crystal structures were deposited to the CCDC with deposition numbers 1967034 (14) and 1967030 (40).

References:

1. APEX3. Ver. 2017.3-0. Bruker AXS Inc., Madison, Wisconsin, USA, 2017.

2. L. Krause, R. Herbst-Irmer, G. M. Sheldrick and D. Stalke, J. Appl. Cryst. 2015, 48, 3-10.

3. G. M. Sheldrick, Acta Cryst. A 2015, 71, 3-8.5.

4. G. M. Sheldrick, Acta Cryst. C 2015, 71, 3-8.6.

Table S1: Summary of XRD Parameter of compound $\mathbf{1 4}$ and $\mathbf{4 0}$

\begin{tabular}{l|c|c}
\hline Compound number & $\mathbf{1 4}$ & $\mathbf{4 0}$ \\
\hline CCDC & 1967034 & 1967030 \\
\hline Moiety Formula & $\mathrm{C}_{15} \mathrm{H}_{13} \mathrm{NO}_{3}$ & $\mathrm{C}_{17} \mathrm{H}_{15} \mathrm{NO}_{3}$ \\
\hline UNIT Formula & $\mathrm{C}_{15} \mathrm{H}_{13} \mathrm{NO}_{3}$ & $\mathrm{C}_{17} \mathrm{H}_{15} \mathrm{NO}_{3}$ \\
\hline UNIT Mw & 255.26 & 281.30 \\
\hline Space group & $\mathrm{P} 121 / \mathrm{c} 1$ & $\mathrm{P} 121 / \mathrm{n}$ \\
\hline Unit cell & & \\
\hline $\mathrm{a}(\AA)$ & $5.1388(3)$ & $10.6981(13)$ \\
\hline $\mathrm{b}(\AA)$ & $19.7529(12)$ & $5.3875(6)$ \\
\hline $\mathrm{c}(\AA)$ & $11.7294(8)$ & $22.901(3)$ \\
\hline$\alpha\left({ }^{\circ}\right)$ & 90.000 & 90.000 \\
\hline$\beta\left({ }^{\circ}\right)$ & $100.519(2)$ & $100.847(3)$ \\
\hline$\gamma\left({ }^{\circ}\right)$ & 90.000 & 90.000 \\
\hline Volume $\left(\AA^{3}\right)$ & $1170.60(13)$ & $1296.3(3)$ \\
\hline $\mathrm{Z}$ & 4 & 4 \\
\hline Reflections & & 15921 \\
\hline Total & 15099 & 5152 \\
\hline Unique & 3425 & 0.64 \\
\hline Resolution $(\AA)$ & 0.71 & 99.7 \\
\hline Completeness $(\%)$ & 99.8 & 2.06 \\
\hline $\mathrm{R}_{\text {int }}(\%)$ & 3.54 & 2.48 \\
\hline $\mathrm{R}_{\text {sig }}(\%)$ & 3.02 & 4.16 \\
\hline Structure Refinement & & 12.1 \\
\hline $\mathrm{R} 1(\%)$ & 4.15 & 1.031 \\
\hline wR2 $(\%)$ & 11.3 & \\
\hline GOF & 1.031 & \\
\hline & & \\
\hline & & \\
\hline
\end{tabular}

